NOSTOCK

UCRL -52930

Vol. 2

i)

\title{
ENVIRONMENTAL EFFECTS OF ENERGY PRODUCTION AND UTILIZATION IN THE U.S. VOLUME 2; PUBLIC HEALTH EFFECTS
}

Complled and edited by Herbert . Newkirk

June I, 1976

Prepared for U.S. Energy Research \& Development

Administration under contract No. W-7405-Eng-48

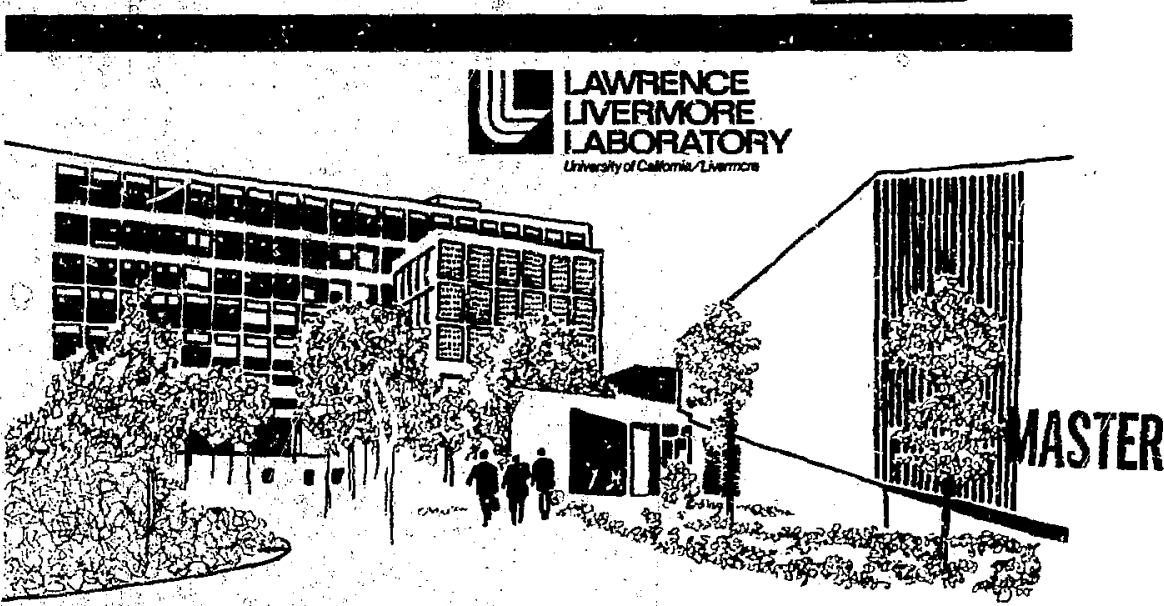

41. 


\section{YOTICE}

"Ibis teport wa prepared is en sccount of work eponsored by the United States Goveramert. Neilher the Untied States nor the United Stales Energy R ssewrth \& Development Administistion, nor sny of thetr eniphyeets nor iny of their contraciors, subcontrectors, of: their employem, imakes env wrranty. express or implied, or assumen eny legal liability or responshility for the aceuracy. compleiteness or pefutness of any iaformation, cpparatus, produet of proceus disclosed, or represents nit its use would not infrines privatistowited rithts."

Reference to company or product name do not implys approval or secommendation of the product by the University of Californis or the U.S. Energy Research \& Development Administration to the exclusion of otheres that may be suitable.

Pristed in the United States of America

$$
\text { Available from }
$$

National Technical Information Service

U.S. Department of Commerce

\section{Port Royal Road}

Springfield, VA 2216!

Price: Printed Copy S ; Microficine \$2.25

\begin{tabular}{|c|c|c|c|}
\hline Pere Ran & $\begin{array}{l}\text { Domeatic } \\
\text { Price } \\
\end{array}$ & Paga Ranos & $\begin{array}{c}\text { Dommetic } \\
\text { Price }\end{array}$ \\
\hline $001-025$ & $\$ 3.50$ & $32 c-350$ & 10.00 \\
\hline $026-050$ & 4.00 & $351-375$ & 10.50 \\
\hline $051-075$ & 4.50 & $376-400$ & 10.75 \\
\hline $076-100$ & 5,00 & $401-425$ & 11.00 \\
\hline $101-125$ & $-5,5,5.50$ & $426-450$ & 11.75 \\
\hline $126-150$ & $5 \leqslant 06 . \Delta \mathrm{D}$ & $451-475$ & 12.00 \\
\hline $151-175$ & 6006.25 & $476-500$ & 12.50 \\
\hline $176-200$ & 7,50 & $501-525$ & 12.75 \\
\hline $201-225$ & 7.75 & $526-550$ & 13.00 \\
\hline $226-250$ & 8.00 & $551-575$ & 13.50 \\
\hline $251-275$ & 9.00 & $576-600$ & 13.75 \\
\hline $276-300$ & 9.25 & $601-4 \mathrm{p}$ & . \\
\hline $301-325$ & 9.75 & & \\
\hline
\end{tabular}

Had $\$ 2.50$ fot ach gdditional 100 poge increment from 601 to 1,000 pagea: add \$1.50 for each additional livd page increment over 1,000 pages. 


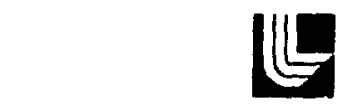

\section{LAWRENCE LNEAMORE LABOAATORY}

Universtyol Caltoma Lvermore Cationsa 94550

\section{ENVIRONMENTAL EFFECTS OF ENERGY PRODUCTION AND UTILIZATION IN THE U.S. VOLUME 2: PUBLIC HEALTH EFFECTS}

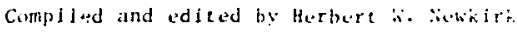

Mis. dace: Jume 1, lgin

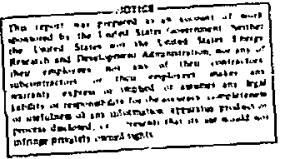




\section{Foreword}

Historlcally, concern in the Unled states over resourcess has focused on Hether or not there would be enough food, energy, and materials to meet current and fucure necds. Today, there is also concern about the ability of oar land. if , and water to absorb all the offluents that energy product lon and ut 14 batcon generates. The firse annual finvirongental quality report accurately epiconize: thls fact: "The ronfli-ts between the consumption patcerns thil we all kant, and the environgental 111 effects that we all wish (a :Ivold, is sharber for energy than perhaps any other aspect of natural resource use."

The issues being debited are complex, and while the writcen pieces on the subfect number in the thousands, to our knowledge there is not yet available a wamat; of the facts related to the environthental effects of energy probuction abd utfizatfon. We have actempted to complle this relevant information and publish it in three volines. It fs anticipaced that these handbooks wil provide a data bink of envlronmencal information that will be helpful in moking the many critical cost/beneft tholces that enersy production and at lllzat lon demands.

Volupu I deals with sources (what the emissions are and where they coage irom), trends (quantites of erafsalons and their disperston with $t$ fme), and costs of control (whal it cakes in time, energy, and money to mest minimum standirds). Volume 11 concerns teself with the public health aifects of eltersy production and utilization. Volume 111 summarlzes the various technfques for controlling emissions, technological as well as economic, soctal, and palfelial.

Fach volume is divided into sections deallng with the atmosphere, water, land, and social activicies - each diviston indicating a particular sphere of man's environment affected by energy production and use. The sources of information that were used in this study included textbooks, journat aricles, technical reports, memoranda, letters, and persunal comounicacions. These are cited in the cext at the end of each subsection and on the applicable tables and figures.

Good writing always benefits from both criticisms and suggestions. Comments on this edition are solicited to guide the compiler in subsequent versions of these fact files. 
Finally, in a project of this magnitude many people contribute both their time and talent and deserve spectal recognition. To our typists, Joan Rlen, Betty Irving, VIrg Jaramillo, and Donna Romero, who typed the rough drafts; to Virginia Caswell of LLL's Technical Information Division who performed a superb fob of Ifterature searching in this field; to Kent Curmings for his skill In editing the volumes; and to the LLL staff members who reviewed portions of the rough dratts, Stuart Winter (Energy Planning and Analysis Group), John Tewes (K Division), Hugh Ellsaesser (C Division), David W. Dorn and Carroll Manlinger (Technical Application Group) we extend our thanks. 


\section{Contents}

Glossary . . . . . . . . . . . . . . . . . . . . . . . vi1

I. Summary of Public Health Effects . . . . . . . . . . . . . . . . 1

I1. Afr Emissions... . . . . . . . . . . . . . . . . . . 5

Chemical factors . . . . . . . . . . . . . . . . . 5

Carbon Monoxide . . . . . . . . . . . . . . . . . . 5

References - Carbon Monoxide .............. 6

Nitrogan Oxides ...................... 8

References - Nitrogen oxides ................. 9

Photochemical 0xidants . . . . . . . . . . . . . . . 10

References - Photochemical oxidants ............ Il

Sulfur Oxides . . . . . . . . . . . . . . . . . 14

References - Sulfur Oxides ............... 18

Nonradloactive Particulates . . . . . . . . . . . . . 24

Keferences - Nonradioactive Particulates . . . . . . . 27

Organic lases . . . . . . . . . . . . . . . . . 40

Ref $\_$rences - Organic Cases . . . . . . . . . . . 40

Trace Metals... . . . . . . . . . . . . . . . . 45

References - Trace Metals . . . . . . . . . . . . 49

Hydrogen julfide . . . . . . . . . . . . . . . . . . . 51

References - Hydrogen Sulifide . . . . . . . . . . . 51

Water Vapor . . . . . . . . . . . . . . . . . 53

References - Water Vapor . . . . . . . . . . . . 54

Radiological factors . . . . . . . . . . . . . . . . . 55

Gaseous Radioactivity . . . . . . . . . . . . . . . . 55

References - Gaseous Radioactivity . . . . . . . . . . 56

Radioactive Particulates. . . . . . . . . . . . . 57

References - Radloactive Partlculates . . . . . . . . 58

Physical Factors . . . . . . . . . . . . . . . . . . 59

Thermal Inputs........................ 59

References - Theralal Triuts . . . . . . . . . . . 60

Electromagnetic Emisstors . . . . . . . . . . . . . . 66

References - Electromagnetlc Emissions . . . . . . . . . 74

Noise . . . . . . . . . . . . . . . . . . . 80

References - Noise .................... . 82 


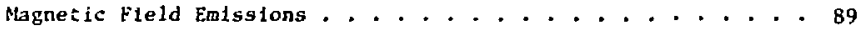

References - Magnetfi Field Enissions . . . . . . . . . 89

X Radiation . . . . . . . . . . . . . . . . . 90

References - X Radiation ................. 93

111. Water Effluents

Chemical Factors ......................... 97

Trace Metals... . . . . . . . . . . . . . . . . 97

References - Trace Metals................ . 100

Radlologlcal factors ... . . . . . . . . . . . . . 103

Tritium . . . . . . . . . . . . . . . . . 103

References - Tricium ................. 103

other kadioactivity . . . . . . . . . . . . . . . 104

References - Other Radioactivity . . . . . . . . . . 104

Physicai factors . . . . . . . . . . . . . . . . . . . . 106

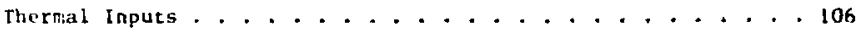

References - Thermal Inputs . . . . . . . . . . 106

IV. Land lise

Chemical factors . . . . . . . . . . . . . . . . . . 108

Acid Fallout... . . . . . . . . . . . . . . . 109

References - Ac ld fallout............... 109

Solld Effluent......................... 110

References - Soldd Effluent ................ 111

Radiological factors . . . . . . . . . . . . . . . . . 112

HLgh-Level Storage...................... . 112

References - High-level Storage . . . . . . . . . . 112

Physlcal factors . . . . . . . . . . . . . . . . . . . . 116

Strip- and Surface-Mining Activities . . . . . . . . . . . I16

References - Sirip- and Surface-Minirg netlvities.... . 11?

Power-Plant Siling. . . . . . . . . . . . . . . . . 119

References - Power-Plant Siting . . . . . . . . . . . 119

Hydroelectric Dams... . . . . . . . . . . . . . . 120

References - Hydroelectric Dams . . . . . . . . . 120 


\section{Glossary}

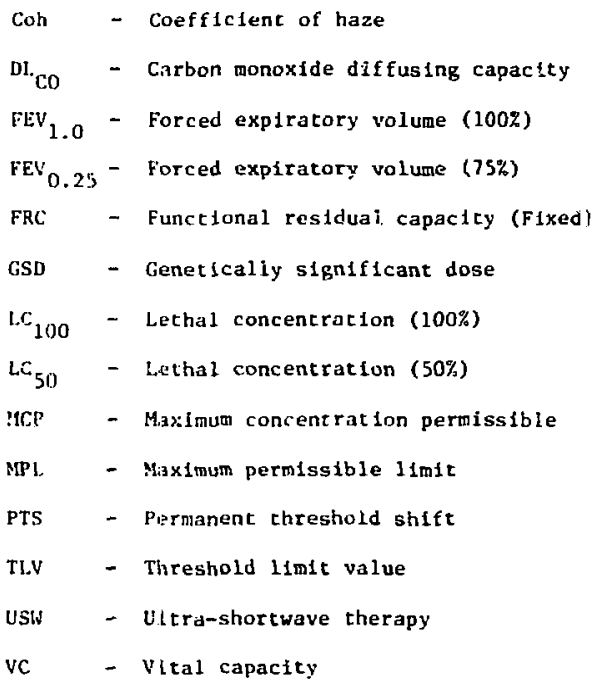




\section{Section I \\ Summary of Public Health Effects}

Although the acute fiffects of emissions on health are easily fientified, It continues to be difficit to establish the presence o: absence of harmfu: effects from permanent exposure to low dosage levels. It is likely that present localized levels ot eulssions in citles aggruvate certain ailments and possibiy faclitate thelr development in susceptible individuals or grups.

We gain the Impression from reading the extensive documentation in this field that there is a tendenc; to exaggerate the associations tetween emisston levels and health effects - making the emIssions seem more hazardous than data supports.

The scientif ic arguments marshalled by opposing sides in debate continue to be so perplexing that there is a tendency to overlook a princtipal objec:ion, simply, that it appears unpleasant. Nevertheless quancitative analysis of the effects of emissions on public health is still scarce, and the work being done on ches: topics is timely. Estimated health effects in 1973 assoclated with electric power production are given in Table 1.

Statistical correlation, the urban factor, and the classical air emission episodes of the Meuse Valley in 1930, Donora in 1948, and London in 1952 coistinue to remain the principal evidence defining health effect: of ajr enissions.

The cruclal question that still remains unanswered is whether of not these effects are noise in ths data or are caused by misfdentification of castlei agents or variations in the susceptiblity of indfviduals. The structure of our present control program continues to rest on the presumption that the latter premise is correct and on the philosophy that susceptible Individuals must be protected.

The hypothesis is still not proven that some atmospheric emissions are mutagenic and increase the death rate from some chronic diseases. How ever, there is consistent statistical evidence from 38 metropolitan areas of the U.S. that ilicreasing concentrations of air emissions and, in partictilar, of nitrogen dioxide and sulfur dioxide, are associated with Increasing death rates from chronic diseases associated with senescence, such as varlous forms of cancer, total cancer, and diseases of the heart. 
Because the Fedelal Clean Asf Act of 1970 acknowledges that the statistical fluctuations of enissions and meteorological conditions can lead to severe episodes of concentrated air emissions with consequent acute illness and mortalicy, tt requires each state to provide an Eplsade Cantral Plan for regions subject to significant discharge, to prevent ambient concentrations Erou reaching levels causing significant harm to health of persons. These levels are given in Table 2. 
Table 1. Estimated health effects in 1973 associaced wich production of electric power.*

\begin{tabular}{|c|c|c|c|c|c|}
\hline Fuel & $k W_{h}(e) \times 10^{9}$ & $\begin{array}{c}\text { Equivalent No. } \\
1000 \text { MW(e) } \\
\text { plants }\end{array}$ & $\begin{array}{l}\text { Estimated } \\
\text { deaths }\end{array}$ & $\begin{array}{l}\text { Estimated } \\
\text { disabilities }\end{array}$ & Unknowns \\
\hline $\operatorname{Coa} 1$ & 846.0 & 128.2 & $2,000-16,000$ & $26,000-39,000$ & $\begin{array}{l}\text { Increased cancer, other } \\
\text { chrontc disease, mutations }\end{array}$ \\
\hline oil & 310.7 & 47.1 & $100-5,000$ & $4,000-9,000$ & $\begin{array}{l}\text { Increased cancer, other } \\
\text { chronic disease, nutations }\end{array}$ \\
\hline Gas & 336.0 & 50.9 & 7 & 700 & $\begin{array}{l}\text { Increased cancer, other } \\
\text { chronic disease, mutations }\end{array}$ \\
\hline Nuslear & 83.3 & 12.6 & $9-20$ & $60-300$ & $\begin{array}{l}\text { Increased risk of } \\
\text { catastrophic accident }\end{array}$ \\
\hline Hyd ro & 271.1 & 41.1 & - & - & $\begin{array}{l}\text { Increased risk from dam } \\
\text { failure accident }\end{array}$ \\
\hline $\begin{array}{l}\text { Wood, waste, } \\
\text { geothermal }\end{array}$ & 2.3 & 0.3 & - & - & - \\
\hline Totals & 1849.4 & 280.2 & $2, ? 00-21,000$ & $31,000-49,000$ & \\
\hline \multicolumn{6}{|c|}{$\begin{array}{l}\text { Approximate annual total deaths in U.S. } \\
\text { Percent associated with electricity production }=0.1-1 \% \\
\text { Approximate number of deaths in U.S. ages } 1-74=1,100,000 \\
\text { Percent associated with electricicy production }=0.2-1.9 \%\end{array}$} \\
\hline
\end{tabular}

*[Hamflton, L. D., and S. C. Morr1s, Brookhaven National Laboratory, Upton, NY, Rept. BNL-19265 (1974).] 
Table 2. Significant levels for episode contro1.*

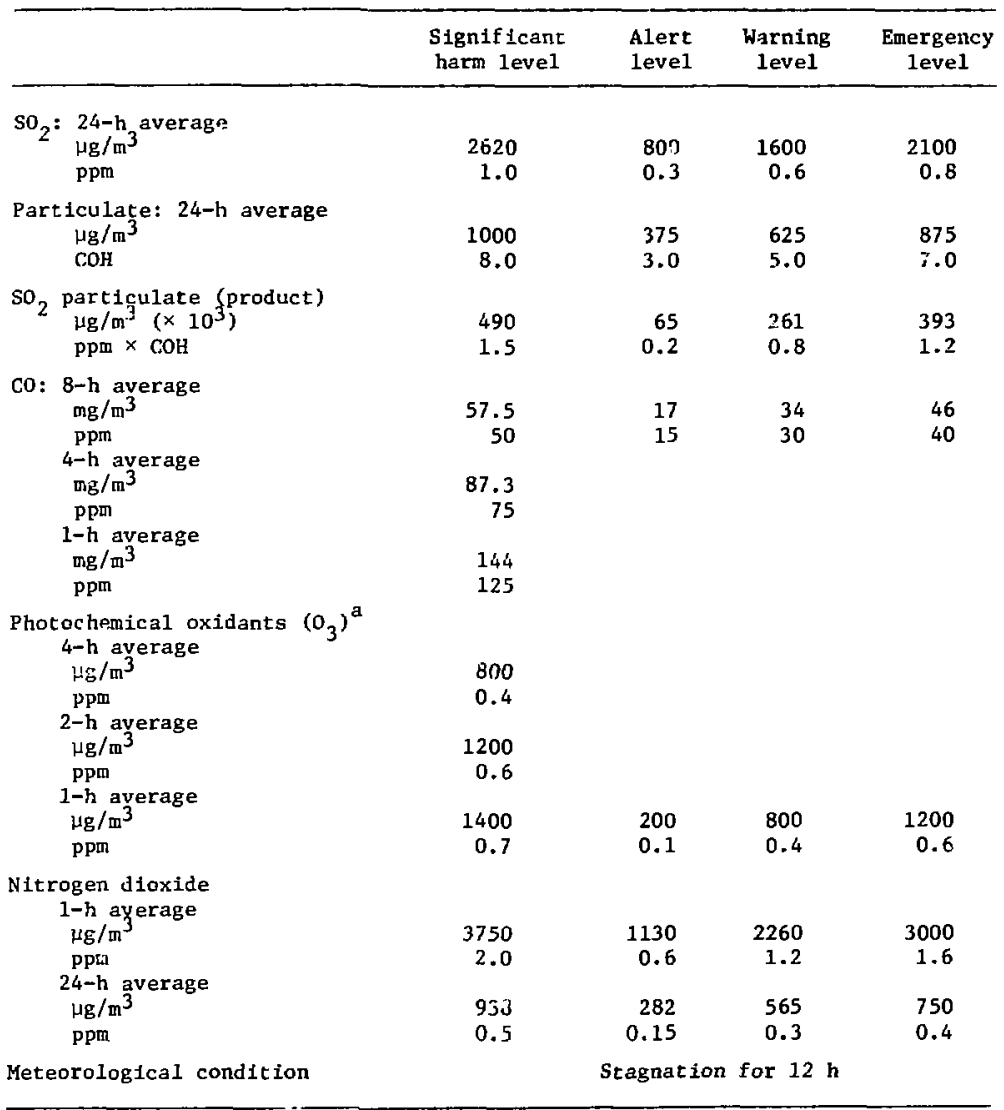

${ }^{a}$ Proposed change to single significant harm level of $1200 \mu \mathrm{g} / \mathrm{m}^{3}(0.60 \mathrm{ppm}), 1-\mathrm{h}$ average, and emergency level of $1000 \mathrm{\mu g} / \mathrm{m}^{3}(0.50 \mathrm{ppm})$.

${ }^{\star}$ [Federal Register 39, March 13, 1974, pp. 9672-9675.] 


\section{Section 11 \\ Air Emissions}

CHEMICAL FACTORS

\section{Carbon Monoxide}

Carbon monoxide combines preferentially with red blood cells to form carboxy hemoglobin. This compound cannot carry oxygen, so the body suffocates. (Fig, 1).

Carbon monoxide is capable of reactjng with other hemoproteins and may Interfere with certain enzyme systems. Performance decrements and adverse cardiovascular effects become evident when there 1 more than $5 \%$ carboxy hemoglobin in the blood. The relationship between carbon monoxide exposure from smoking and carbon monoxide exposure from the amblent air, and the relationship between carbon monoxide exposure and cardlovascular disease, are not clear.

It is accepted by all workers that $20 \%$ carboxyhemoglobin (equivalent to saturation at about $120 \mathrm{ppm}$ carbon monoxide) can cause symptoms and Impair performance but healthy subjects rarely complain of any symptoms with saturations less than $10 \%$ ( $(20 \mathrm{ppm} \mathrm{CO}$ ).

There is little convincing evidence that concentrations of gas giving less than about $8 \%$ saturation ( 48 ppm $C 0$ ) has any effect on perception or "intellectual" performance, and there is evidence that electroencephelogram activity remains unaffected unt1l saturations approach $33 \%$ ( 200 $\mathrm{ppm} \mathrm{CO}$ ).

The results of sound work on normal subjects have shown that saturations of about $5 \%$ ( $(30 \mathrm{ppm} \mathrm{co}$ ) have measurable effects on cardiac functions. For comparison, the present carbon monoxide standards, designed to prevent carpoxyhemoglobin saturations exceeding 2\%, are 9 ppm for $8 \mathrm{~h}$ and $35 \mathrm{ppm}$ for $1 \mathrm{~h}$. 


\section{References - Carbon Monoxtde}

Air Quality Criteria for Carbon Monoxide, Department of Health, Education, and Welfare, National Air Pollution Control Administration, Washington, DC, Publ. AP-62 (1970).

Astrup, P., "Carbon Monoxide and Perlpheral Arterial Disease," J. Clin. Lab. Investigation Suppl. 99, 193 (1967).

Goldsmith, J. R., "Carbon Monoxide Research - Recent and Remote," Arch. Environ. Health 21, 118 (1970).

Eckardt, R. E., H. H. MacFarland, Y. C. E. Alarie, and H. H. Busey, "The Blologlc Effect from Long-Term Exposure of Prinates to Carbon Monoxide," Arch. Environ. Hea1th 25, 381 (1972).

Lawther, P. I., in Proceedings of the Conference on Fuel and the Environment, November 26-29, 1973 (Institute of Fuel, London, 1973), Vo1. 2, p. 123.

Ross1-Fane1li, A., and E. Antoninio, "Studies on the Oxygen and Carbon Monoxide Equilibria of Human Myoglobin," Arch. Biochem. Biophys. 77, 478 (1958).

Theodore, J., R. D. O'Donnell, and K. C. Back, "Toxicological Evaluation of Carbon Monoxide in Humans and Other Mammalian Species," J. Occup. Med. 13, 242 (1971).

Zimmerman, P. W., W. Crocker, and A. E. Hitchcock, "The Effect of Carbon Monoxide on Plants," Contrib. Boyce Thompson Inst. 5, 195 (Apri1-June 1933). 


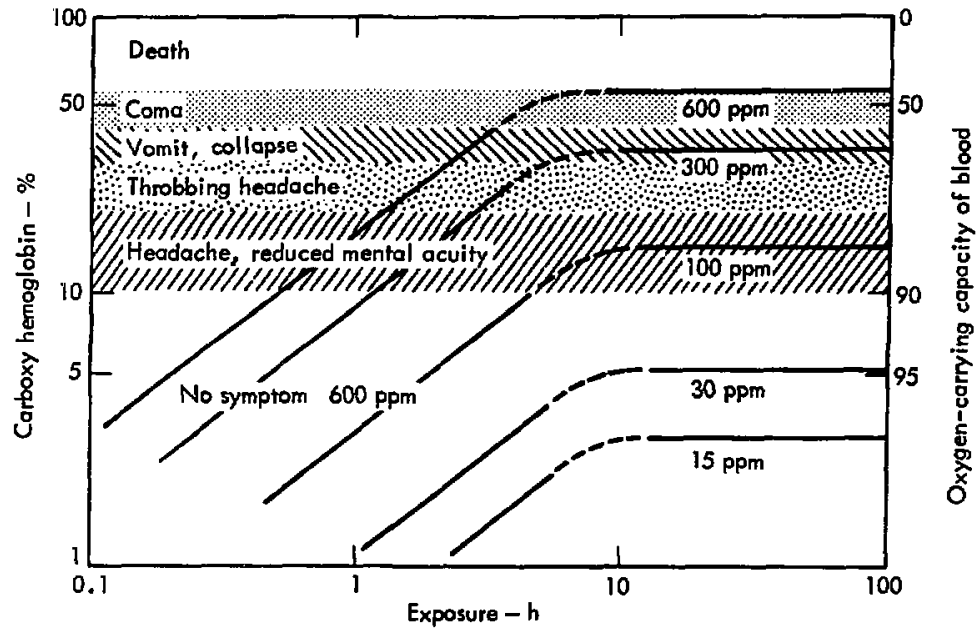

Fig. 1. Carbon monoxide combines preferentially with red blood cells to form carboxy hewo zlobin, which cannot carry oxygen. [Myers, P. S., "Nation's Cost/Benefit Ratio Weighs Heavily on Auto Emissions," SAE Journal (March 1970),] 


\section{N1trogen Oxides}

The significance to human health of currently encountered atmospheric levels of nitrogen dioxide is inadequately known. Very few epidemiological studies have been performed, and these have ylelded conflicting results. In most instances, the studies in Californla have fafled to correlate exposure to oxides of nitrogen with ventilatory dysfunction, respiratory symptoms, chronic lung disease, or mortality.

Although epidemiological data do not offer a definitive answer to the question of whether or not high amblent levels of nitrogen dioxide cause 11 lness, the Chat tanooga study, which bears most directly on this point, suggests that in certatn populations exposure to high ambient concentrations (0.08 ppm) of nitrogen dioxide is associated with an increased $r$ isk of respiratory infection.

However, since more recent data indicate that (1) other pollutants, such as nitrates and sulfates, may have acted in concert with nitrogen dioxide at Chat tanooga; (2) the nitrogen dloxide measurements were in themselves somewhat Inaccurate; (3) follow $\cdots u$ studies of the affected population did not disclose sequelae; and (4) recent epidemiologic studies have not demonstrated illness due to oxidant exposure of similar degree, an increase in the standard to $0.08 \mathrm{ppm}$ on an annul basis would be consistent with this new information.

Definitive evidence demonstrating that exposure to nitrogen dioxide can be deleterious to respiratory functions comes from two sources: (1) acute exposure of humans to high leyels of nitrogen dioxide invarlably results in respiratory disease and (2) experfmental animals exposed to elevated levels of atmospheric nitrogen dioxide develop pathologic changes resembling emphysema and an enhanced susceptibllity to bacterial infection.

A range of adverse effects has been described which correlate with the degree of exposure. Eye and nasal irritation occurs after exposure to 15 ppr of nitrogen dioxide. Pulmonary discomfort is noted at levels of $25 \mathrm{ppm}$, and bronchlolitis with focal pneumonitis occurs after exposures of 25 to 75 ppm of nitrogen dioxide. The duration of these exposures was put at less than $1 \mathrm{~h}$. Comparable short pertods of exposure to 150 to $200 \mathrm{ppm}$ causes fatal pulmonary fibrosis. Higher exposures are associated with acute pulmonary edema, and sometimes death. 
Assessments of the envirommental hazard of nitrogen diox lie can be confined to the pulmonarj effects of the emissions, because in hisans extrapulmonary abnormalities of blologlcal significance have not been recorded, and in experimental animals the lung is the target organ for nitrogen dioxide Induced injury.

The No ${ }_{x}$ effect on the ea:th's ozone shield is based on highly complex, chemical models which at this time remain inadequately tested. Admittediy, several processes known to be significant are ignored; consequently, current results differ in several important respects from avallable observational data. (See the sections on pho'ochemical cxidants and electromagnetic enisstons.)

\section{References - Vitrogen oxides}

Crutzen, P. J., Ambio 3, 201 (1974).

Grobecker, A. J., S. C. Coronit1, and R. H. Cannon, JI. Report of Findings; The Effects of Stratospheric Pollution by Alrcraft (Departient of Transportation Climatic Impact Assessment Program, Washington, DC, 1974). 


\section{Photochemical Oxidants}

Numerous epldemlologlcal studies have been conducted on the effects of photochemlcal oxldants. The only convincing relationships (Table 3) have been those for eye 1rritation, at times accompanied by nasal irritaticil (when oxtdant concentrations exceeded $0.10 \mathrm{ppm}$ ); for aggravation of symptoms among asthma patients (when oxldant concentrations exceejed $0.13 \mathrm{ppm}$ ); and for impalrment of athletic performance (for hourly oxldant concentrations in the range 0.03 to $0.30 \mathrm{ppm}$ ).

No convincing relatlonships were observed between short-term varlations in in oxldant levels and daily mortality or hospltal admissions. Most of the reported studies do suggest, however, that photochemical oxidants are potentially hazardous environmental contaminants.

Recent work demonstrates the synerglstlc effect of ozone and sulfur dioxide enforces the concept that emissions cannot be considered separately when standards are promulgated and that comple: interrelationships between them must be considered. It is also important to know whether phenomena well denonstrated in animals, such as tolerance or cross protection whereby exposure to ozone provides some protection to other lrritant oxidaris, occur in human belngs.

Fhotochemical oxidants tend to exert their toxic effect on the body through inhalation. Alterations in the pulmonary function of the lungs are among the effect, Other effects include pathological, chemical, and blologlcal changes in the lungs and other organs.

Occupational exposure to ozone has been documented by several stujles; however, rany of the studies are compllcated by the presence of einissions other than ozone. Most of the studles were on subjects exposed to ozone concentrations on the order of $0.3 \mathrm{ppm}$ and produced no convincing evidence that functional Impairment was due to ozone; further studies should be conducted.

Laboratory experiments have shown more def initive results. The threshold level at which nasal and throat irritation will result in humans under prolonged exposure to ozone appears to be about $590 \mu \mathrm{g} / \mathrm{m}^{3}(0.3 \mathrm{ppm})$. For short-term exposures there is no firm information conceinlng exposure under $980 \mathrm{\mu B} / \mathrm{m}^{3}(0.5 \mathrm{ppm})$. Exposure to concentrat lons of 980 to $1960 \mathrm{\mu g} / \mathrm{m}^{3}$ $(0.5$ co $1.0 \mathrm{ppm})$ for periods of 1 to $2 \mathrm{~h}$ produces changes in the pulmonary function: Increased al ruay resistance, decreased vital capacity, decreased 
carbon monoxide diffusing capacity, and decreased forced expiratcry volume (Fig. 2). Concentrations in the range of 1960 to $5900 \mathrm{\mu g} / \mathrm{m}^{3}(1.0$ to $3.0 \mathrm{ppm})$ produce extreme fatigue and lack of coordination ia enme people.

\section{References - Photochemical Oxidants}

Barrett, L. B., and T. E. Wadde11, Cost of A1r Pollution Damage, Environmental Protection Agency, Research Triangle Park, NC, Publ. AP-85 (1973).

Conference on Health Effects of Air Pollution, Summary of Proceedings (USGPO, Washington, $\overline{D C}, 1973$ ).

Downing, P. B., Environ. Affairs 4, 711 (Fall 1975).

Ellsaesser, H. W., "A Reassessment of Stratospheric Ozone - Credibility of the Threat," paper to be presented at the symposium on Stratospheric Pollution and Skin Cancer at the 50th Anniversary Congress of the Pan American Medical Assactation, October 24-29, 1976, Hollywood, FL.

Goldsnith, J. R., and J. A. Nadel, J. Air Poll. Cont. Assoc. 19, 329 (1969). MeCarrol1, J., Califi. Afr Environment 4 (Winter 1974).

Zelac, R. E., et al., Environ. Sc1. 4, 262 (1971); 4, 325 (1971). 
Table 3. Summary of selected health effects on exposure to photochemical oxidant . *

Extent and duration of exposure

Observed effects

A. Demonstrated adverse effects

0.1 ppm and above

$0.2 \mathrm{ppm}$ for $3 \mathrm{~h}$

0.25 ppm daily maximum hourly exposure

$0.25-0.30$ ppm da1ly maximum hourly exposure

0.3 to 0.8 ppm average for 8-h workday

$0.37 \mathrm{ppm}$ for $2 \mathrm{~h}$
Eye Irritation in men

Decreased visual activity for dark adaptation and middle vision ranges in man

Increased frequency of asthmatlc attacks In man

"Threshold" for Increased coughing and chest discomfort in man

Complaints of chest discomfort and throat Irritation by workers

Decreased pulmonary function after moderate exerctse in man

B. No demonstrated adverse effects

$0,13 \mathrm{ppm}$ dally maximum hourly exposure

$0.05-0.28 \mathrm{ppm}$ daily

average exposure

$0.2-0.25$ ppm average

for 8-h workday

Arbient concentrations In Los Angeles
No significant Increase in asthmat ic attacks in man

No effect on ventilatory performance of school children

No complaincs of chest discomfurt and throat Irritation of workers

No significant effects on mortalfty due to occurrence of smog-alert days in man

“[Downing, P. B., Environ. Affairs 4, 738 (Fall 1975), reprinced by permission.] 


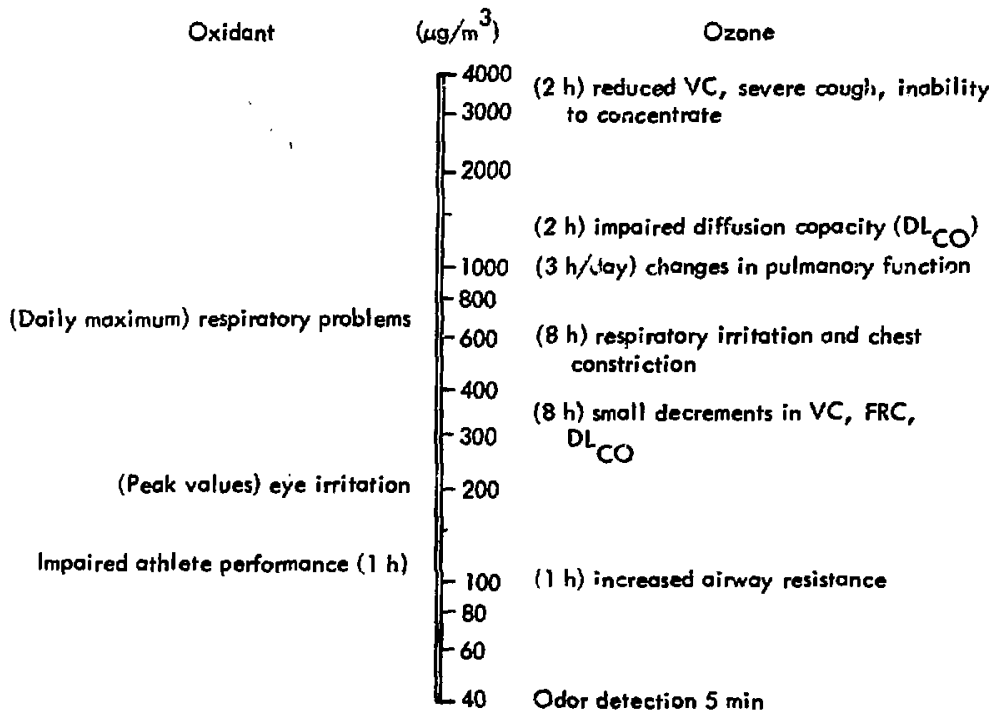

Fig. 2. Alr-qualic; criteria for photochemical oxidants. Conversion of effects data co criterda. (Lawrence Ilvermore Laboratory, staff compilation.] 


\section{Sulfur oxides}

While energy needs have increased and clean sources of energy are limfed, any reduction in standards for $\mathrm{SO}_{2}$ and particulates that permits increased community emissions and concentrations wlll certainly result in a siguificant increase in the number of people who may become 111 and die (Figs, 3, 4 and 5). If standards are not met, the excess number of asthma attacks have been estimated to be 6 million to 10 million each year, and could total over 50 million during the years 1975 through 1980.

The levels of $\mathrm{SO}_{2}$ which are present in large urban areas appear to expose significant numbers of people to health risk during conditions of temperature inversion and air stagnation. Nasal mucus flow rate, alrway resistance, and subjective response were studfed in 15 young men during 6-h exposures to 1 , 5, and $25 \mathrm{ppm}$ sulfur dioxide. A significant decrease in nasal mucus flow rate during the 5- and 25-ppm exposures was observed. This decrease was greatest in the anterior nose and in subjects with an initially slow mucus flow rate. Pharyngea $I$ air samples ylelded less than $1 \%$ of the $\mathrm{SO}_{2}$ inhaled, even after a 6-h exposure to $25 \mathrm{ppr}^{\mathrm{SO}_{2}}$. An increased nasal airflow resistance and a fall in forced expiratcry volume in 1 s and forced expiratory flow during the middle half of expired volume was found at all exposure levels, but there was no change in "closing volume." Discomfort was proportional to $\mathrm{SO}_{2}$ concentration, but never excessive. Subjects with initially slow nasal mucus flow rates experienced the greatest discomfort.

Evidence has now begun to appear that significant drops in enission levels in recent years have not been accompanted by reductions in respiratory death rates. A recent study of $\mathrm{SO}_{2}$ in New York City is informative. It found that during the period 1963 to 1972 the average level of $\mathrm{SO}_{2}$ declined $60 \%$ (i.e., 2-i/2-fold). Over the same perlod there was no decline in death ratss in any of the diseases usually attributed to $\mathrm{SO}_{2}$. But even more surpris:ng, it found that the fraction of excess deaths "explained" by regression on $\mathrm{SO}_{2}$ concentrations doubled. This seens to inply that $\mathrm{SO}_{2}$ at the more recent levels is 5 times as lethal, yet is still causing the same death rats as when the mean level was $2-1 / 2$ times higher.

In the Harlem area of New York City, no relationship between dafly visits to emergency ths and daily levels of elther sulfate or sulfur dioxide have been observed, whereas in the Bedford-stuyvesant area of Brooklyn, a relatively strong correlation between daily vistts for asthma and dafly levels of sulfur dloxide but not with sulfate have been observed over and above the 
effect of temperature. In both areas there was a strong relationship between daily visits for astria and the first cold spells of the Fall season. The average male/femaie rat to for asthma visits to hospital emergency rooms of both areas in the age group under 13 years of age was 1.7 and for over 13 years of age group was 0.6 .

It appearg from the data that Brooklyn presents a different picture for the environmental influence on asthma attacks. Even though it has been shown by previous investigators that asthratics are sensitive to sulfur doxide, we feei that in our case sulfur dioxide is not necessarily the causative agent, but might rather implicate some other confounding variable (most likely an environmental agent) that is disseminated in the general Bedford-Stuyvesant. area in a day-to-day pattern similar to sulfur dioxide. We infer this from the fact that levels of sulfur dioxide were somewhat higher on the average in the Harlem area than they were in the Brooklyn area.

New York City hospitals reported a rise In respiratory Infections and cardiac diseases during a 10-day perlod with dally 0.07 to $0.86 \mathrm{Ppm} \mathrm{SO}_{2}$; the rise In hospital admissions was evident before $0.25 \mathrm{ppm}$ was resched. A simllar occurrence was noted in Rotcerdam during the few days with 0.11 to $0.19 \mathrm{Ppm} \mathrm{SO}{ }_{2}$

As for long-term studies, one in Genoa, Italy, demonstrated a higher incldence of resptratory disease in an area wich an annual mean of $0.092 \mathrm{ppm}$ $\mathrm{SO}_{2}$ vs arers with annual means of $0.037 \mathrm{ppm}$ and $0.028 \mathrm{pgm}$, respectively. Another study, done In areas near a common suelter, demonstrated again the correlation between $\mathrm{SO}_{2}$ concentration and respiratory symptoms; after the smeiter Installed a higher stack for better dispersion, the respiratory symptoms disappeared.

Sulfur dloxide, particulates, and, even m ine, their products - sulfuric acid and acid sulfates - seriously affect the health of significant numbers of hulaans, even a. the levels currently consldered to be safe. The major target organs are the heart and lungs; therefore, large numbers of individuals in the population are in a high-rlsk category regardless of the source of stress. These emissions may act over long periods of tme to produce respiratory tract changes in normal individuals. Their major acute or short-term action appears to be as disease and death accelerators, significantiy shortening the lives of those who have poor adaptive capacttjes. 
Studies of episodes suggest that a rise in the daily death rate occurred when the concentrations of sulfur dioxide rose abruptly to levels at or about $715 \mu \mathrm{g} / \mathrm{m}^{3}$. Daily concentrations of sulfur dioxide $\mathrm{In}$ excess of $1500 \mu \mathrm{g} / \mathrm{m}^{3}$ for 1 day in conjunction with levels of suspended particles exceeding 2000 $\mu g / m^{3}$ appear to have been assoclated with an increase in the death rate of $20 \%$ or more cver baseline levels. This same effect has been observed at lower sulfur dioxide levels when the maximum emission levels lasted for longer and shorter periods.

At concentrations ranging from 300 to $500 \mu \mathrm{g} / \mathrm{m}^{3}$ of sulfur dioxide (24-h mean), with low particulate levels, Increased hospital admissions of clder persons for respiratory disease have been noted; absenteeism from work, particularly with older persons, also has been noted. At lower levels, i.e., at concentrations as low as 105 to $120 \mu \mathrm{g}^{3}{ }^{3}$, there have been observations of increased frequency and severity of respiratory symptoms and lung disease.

Studies carried out in four distinct geographic areas of the U.S. Involving 20,472 children and adults clearly linked excessive, acute respiratory disease with communitles heavily concentrated with sulfur dioxide and suspended sulfates (Table 4). Exposure of 3 years or longer was an Important component in Increased $r i s k$ for acute respiratory disease. Laryngotracheobronchitis was the major condition productive of excess, acute, lower-respiratory disease in children in heavily concentrated communities in the Salt Lake Basin and Rocky Mountain communities. The effect of emisslons o. excess, acute respiratory disease was found to be independent of socioeconomic status and cigarette smoking.

The levels of air emissions for sulfur compounds and partizulates decreased somewhat between two sampling periods 5 to 6 years apart in Rer1in, New Hampshire. Population surveys showed that there was a lower prevalence of respltatory symptoms in 1967 than in 1961. These differences persisted after age standardization anci when comparisons were made within a smoking category. Changes in Inhaling habits or the use of filter-tip cigarettes did not seen to be likely explanations for this decrease in prevalence. Tests of puimonary function were less clear cut but did indicate that a slight improvement in pulmonary function had possibly occurred. It is belleved that these changes may be related to the decrease in the levels of air emissions.

At sulfuric acid concentrations of $1100 \mu \mathrm{g} / \mathrm{m}^{3}$, workers and exposed volunteers immediately experienced irritation of the throat and nose. At 2400 - to $6000-\mu \mathrm{g} / \mathrm{m}^{3}$ sulfurlc acld concentrations, all subjects had severe 
throat Irritation and cough. Sulfur dioxide, on the other hand, will not cause such symptoms in most humans even at concentrations of $13,400 \mu \mathrm{g} / \mathrm{m}^{3}$ (5 ppm). Sulfuric acid levels of $500 \mu \mathrm{g} / \mathrm{a}^{3}$ provoked rapid increases in the respiratory rate, and the effect continued for several ninutes after exposure.

A study of asthmatics carried out in the Metropolftan New York area showed that when minimum temperatures were 30 to $j 0^{\circ} \mathrm{F}$, dose-related increments in asthma attack rates were associated with increments in total suspended particulates and suspended sulfates but not sulfur dioxide. The threshold level for morbidity excess occurred at daily sulfate levels of $7.3 \mu \mathrm{g} / \mathrm{m}^{3}$ when minimum temperature exceeded $50^{\circ} \mathrm{F}$, while the excess morbidity threshold increased to $12 \mu \mathrm{g} / \mathrm{m}^{3}$ sulfate when minimum temperatures were -2 to $10^{\circ} \mathrm{C}$ (30 to $50^{\circ} \mathrm{F}$ ). No firm evidence could be found to associate elevations in sulfur dioxide ( 100 to $180 \mu \mathrm{g} / \mathrm{m}^{3}$ ) with excessive asthma attack rates on elther cold or warm days. A linear regression of asthma attack rate on temperature showed that temperature alone had little effect on asthma attack rates.

Adverse health effects are associated principally (80\%) with the particulate measure (smoke shade); only a small fraction (20\%) are associated with the $\mathrm{SO}_{2}$ measure (Table 5 ).

If we regress mortality on temperature and $\mathrm{SO}_{2}$ alone, the effects attributable to the $\mathrm{SO}_{2}$ measure are increased approximately threefold. This shows that $\mathrm{SO}_{2}$ serves as an index of particulate concentration and, presumably, of volatile emissions also. Moveover, it shows that other mortality and morbidity studies which use $\mathrm{SO}_{2}$ as a princlpal measure of air emissions are undoubtedly measuring the effects of particulates, or even of other volatile components, rather than those of $\mathrm{SO}_{2}$.

Within the limits of the accuracy of the data, despite a $70 \%$ reduction in $\mathrm{SO}_{2}$ levels in the latter perlod (1970-1972) as compared to the earlier years, there has been no reduction in the adverse health effects assoclated with $\mathrm{SO}_{2}$ measure. This tends to confirm that $\mathrm{SO}_{2}$ is serving as a day-to-day indicator of air quality rather than functioning as a harmful emission.

Dafly emission levels reflect the joint effects of the volume of emissions which are emitted into the atmosphere, and meteorological conditions. 
Whether fuels contain $0.3 \%$ sulfur or $3 \%$ sulfur, the daily so $_{2}$ level is responsive to these joint effects. Of course, its absolute level will be much lower $1 \mathrm{x}$ low sulfur fuel is used; however, this would not necessarily result in a reduction of adverse health effects unless $\mathrm{SO}_{2}$ was itself a harmful emfssion.

Statistical studies suggest that $\mathrm{SO}_{2}$ is not only serving as an indicator of air quality but also is not injurious, at least at the ambient levels encountered in New York City in the $1960^{\prime} \mathrm{s}$ - namely, average levels of $0.2 \mathrm{ppm}$ and peak levels of $0.6 \mathrm{ppm}$.

\section{References - Sulfur oxides}

Air Qualicy Ciriteria for Sulfur oxides, Department of Health, Education, and Welfare, Nat Ional Air Pollution Control Administration, Washington, DC, Publication AP-50 (1970).

Andersen, H., Arch. Environ. Health 28, 31 (1974).

Battige11i, M. C., Environ. Occup. Med. 10, 500 (1968).

Carnow, B. W., Calif. Air Environment 4, 7 (1974).

Conservation and Efficient Use of Energy (Part 2), Joint Hearings in Government * Operations and Science \& Astranautics, 93rd Congress, July 10, 1973, USGPO, Washington, DC.

Cost of Clean A1r, Annual Report of the Administrator of the EPA, October, 1973, 93rd Congress, 12th Session, Doc. No. 93-40, USGPo, Washington, Dr.

Electrical World, Staft Report (August 1, 1974), p. 25.

Ferris, B. G., et al., Arch Environ. Health 27, 179 (1973).

French, J. G., et al., Arch. Environ. Health 27, 129 (1973).

Goldscein, J. F., and G. Block, J. A1r Poll. Cont. Assoc. 24, 665 (1974).

Lawther, P. J., In Proceedings of the Conference on Fuel and the Environment, November 26-29, 1973 (Institute of Fuel, London, 1973), Vol. 2, p. 123 .

Middleton, J. T., "A1r Pollution Does Threaten Human Health," National Tuberculosis and Respiratory Diseases Assoc. Bul1. (February $1 \overline{971)}$.

Moran, J. P., private communication (1974).

Schinmel, H., T. J. Murowski, and N. Gutfeld, "Relation of Pollution to Mortality, New York C1ty, 1963-1972," paper presented at the Annual Meetiug of the Air Pol:uition Control Association, Denver, Co, June 9-13, 1974. 
Table 4. Summary of findings In CHESS pulmonary function studies.*

Location Time Age group tested Findings

Cincinnati 1967-1968 Second grade White children exposed to average suspended sulfate levels of 9.5 $\mu \mathrm{g} / \mathrm{m}^{\mathrm{j}}$ had lower $\mathrm{FEV}_{0.75}$ than white chilo:en exposed to average suspended sulfate levels of 8.3 $\mu g / \mathrm{m}^{3}$

The FEV 0.75 of black and white children was lowest in winter

The FEV 0.75 of black chfldren dfd not vary with air emission exposure

The FEV 0.75 of black chIldren was consi itently lower than that of whites

New York 1970-1971 A11 elementary grades

The $\mathrm{FEV}_{0.75}$ of white children aged 9 through 13 years, who had beer exposed to high levels of sulfur oxides and particulates during the first decade of life, was lower than that of children who had not been so exposed.

This finding was statistically sfgnificant in males, but not in females

The FEV $_{0.75}$ of children aged 5 through 8 years did not vary consistently with emission exposure

The FEV 0.75 of children in all grades was lowest in winter

* Health Consequence of Sulfur Oxide: A Report from CHESS 1970-1971, Envirommental Protection Agency, Research Triangle Park, NC, Publ. EPA-650/1-74-004 (1974).] 
Table 5. Estimates of "g premature deaths" associated with air emission, New York City, 1963-1972." "\% premature deaths" is a sensitive indicator of adverse healch effects. Adverse healch effects are assoclated principally $(80 \%)$ with the particulate measure (smoke shade) and only a small fraction (20\%) with $\mathrm{SO}_{2} \cdot \dot{*}$

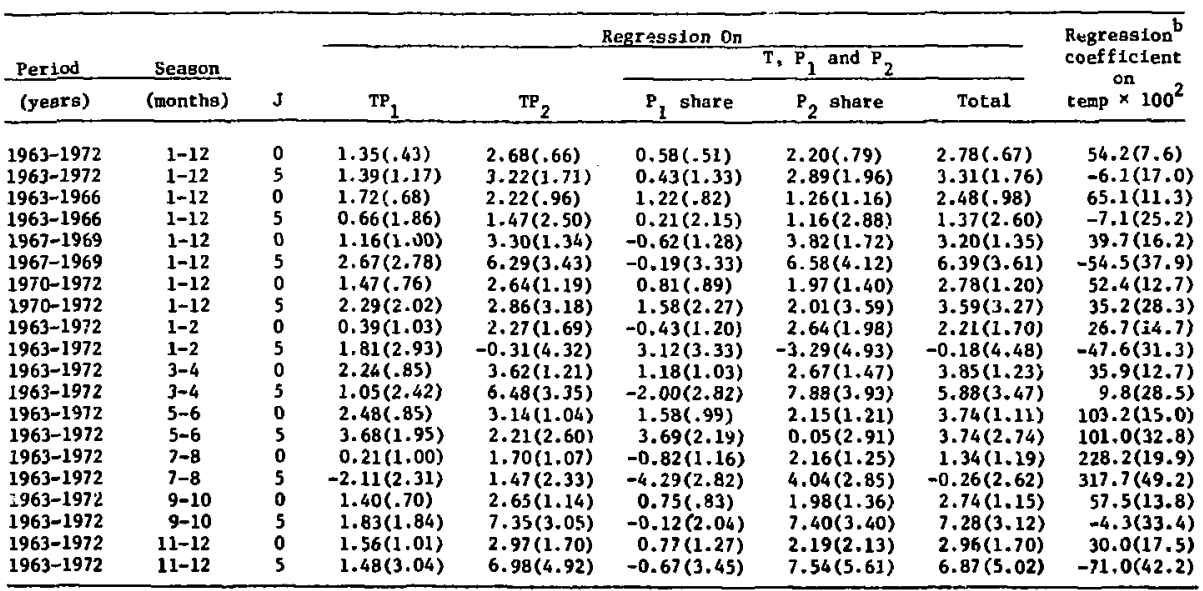

abtalned by regressing cocal dally mortality on geasonally corrected mean dally teaperature $(\tau), \mathrm{SO}_{2}\left(\mathrm{P}_{1}\right)$, and moke shade $\left(P_{2}\right)$. Figure in parentheses is the standard error of the eatiante. Two estisates are given, one

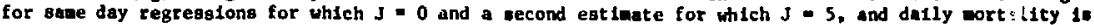
regressed on same day and five previous days of $T, P_{1}$, and $P_{2}$. See or18inul study for discusalon of methode and qualificatione [Schimel, $H$. , and $L$. Greenburg. " $A$ Study of the Relation of Pollution io Hortelity, Neu York City, 1963-1968," J. Alr Poll. Cont. Aasoc. 22: 607 (1972)\}.

b This information has been included to show the coaplexity of wortality, temperature, and ealsotion relationships. In a previous gtuiy when lagged cemperaturea were not included the "percent premature death" estimate with lags was approximately double that of the sine day estioate (aane reference an above).

"[Schimmel, H., and T. J. MurawskI, J. A1r Poll. Cont. Assoc. 25, 739 (1975).] 


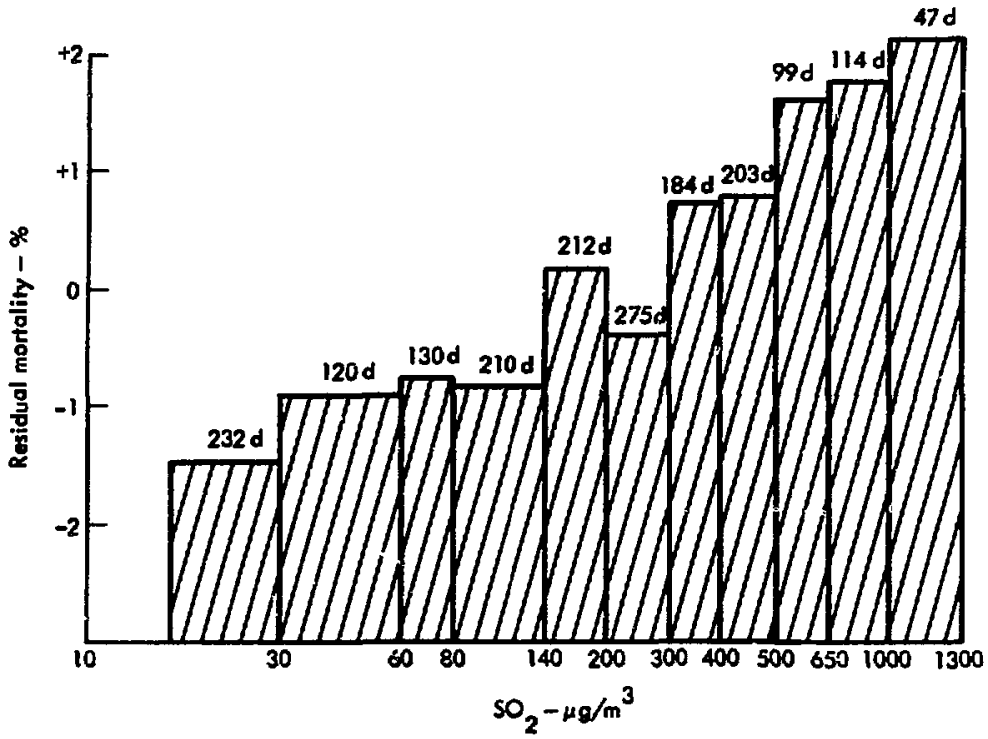

F18. 3. Mean realduel wortality by 502 elagelfication, Hew Jersey Metropolican Area, 1962-1966. H1gh $\mathrm{SO}_{2}$ days differ from 10 s $\mathrm{SO}_{2}$ deye in many ways. Which of these differences is responsible for the correlation with nortality reanins ro be deterwined. [Buechley et. 브.. Arch. Envitron. Health 27, 137 (1973), copyr1ght 1973, Anerican Fadical ineociation.] 


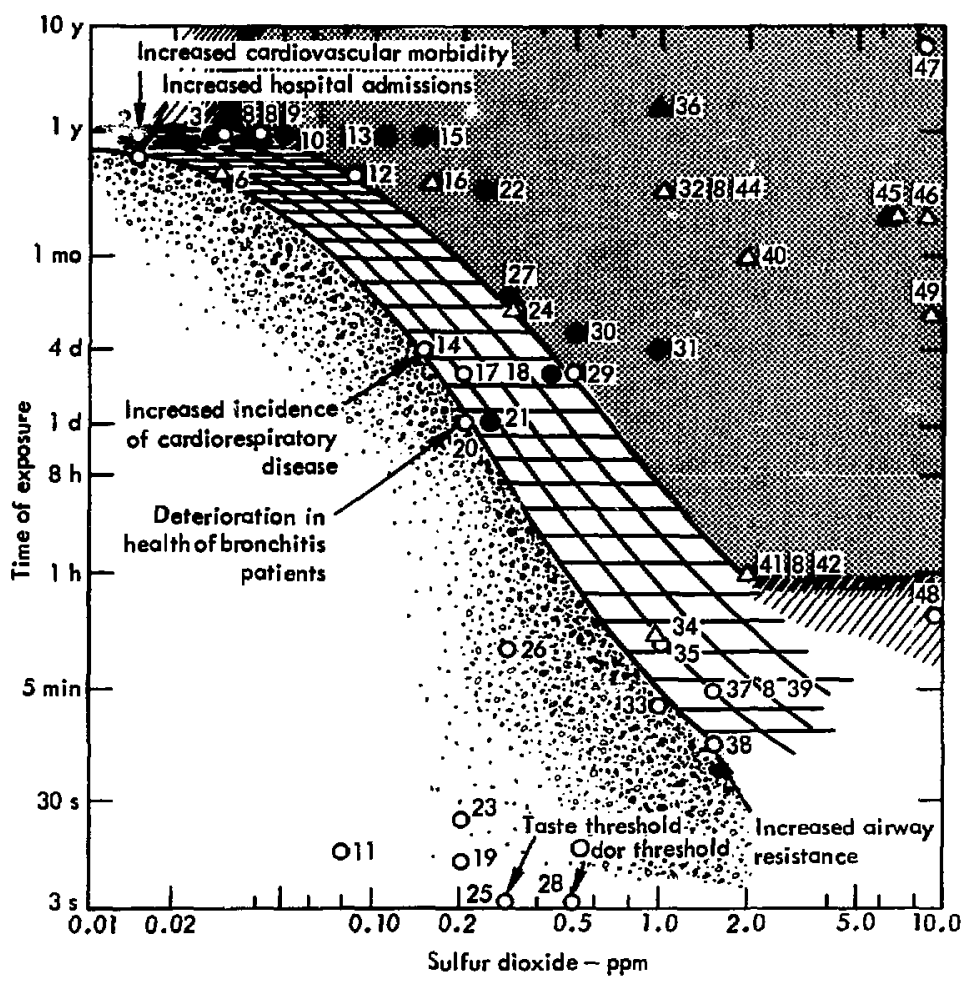

FIg. 4. Effects of sulfur oxides on health. The numbers refer to specific incidents, for example, the London, England, smog of 1952 is represented by point 31 (152 deaths). (o) morbidity in man; (๑) mortality in man; $(\Delta)$ morbidity in animals; ( $\Delta$ ) mortality in animals. The hatched area indicates the range of concentrations and exposure times in which deaths have been reported in excess of tormal expectation. The crosshatched area indicates the range of concentrations and exposure times in which significant health effects have been reported. The stippled area indicates the ranges of concentrations and exposure time in which health effects are suspected. This is the upper bound of the error envelope. [From U.S. Department of Health, Education, and Welfare, Pub1. No. 1619, USGPO, Washington, DC.] 


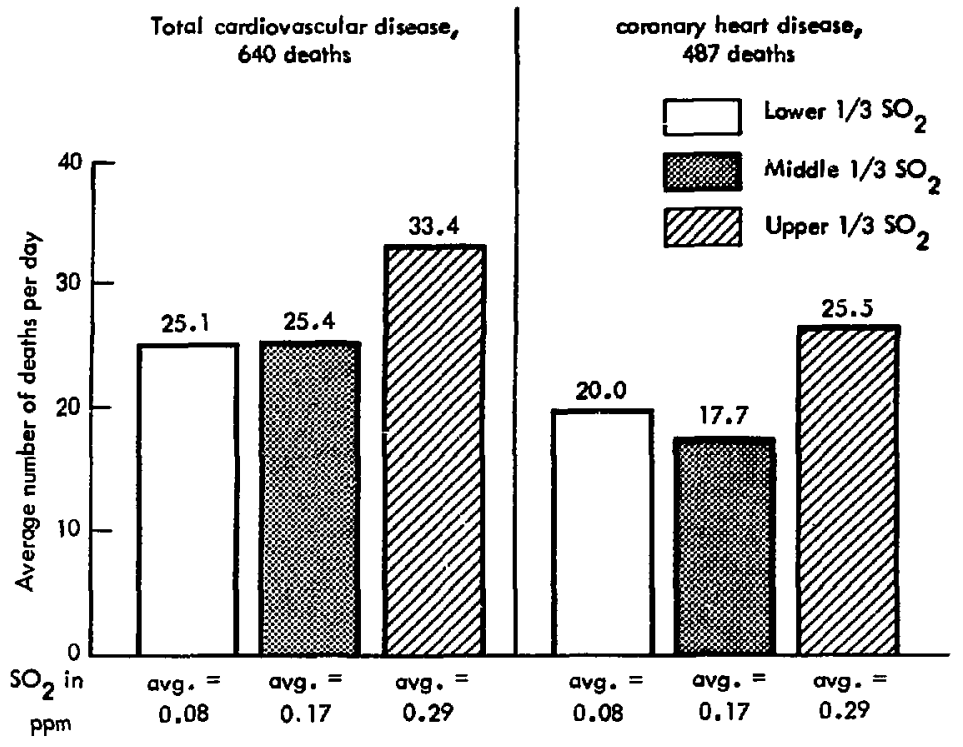

$\mathrm{Fig}$. 5. Deaths due to cardiovascular disease and levels of $\mathrm{SO}_{2}$ (averaged over $212 \mathrm{mi}^{2}$ ) in males 55 and over, Chicago, January 1966 . The $\mathrm{SO}_{2}$ averages shown are derived from the $24-\mathrm{h} \mathrm{SO}_{2}$ readings (ppa), as monitored by the city. The significant increases in cardiac disease and the absence of any increase in other disease categorles further support the validity of these studies, since those suffering from cardlac difficulties would be expected to be the most affected during high atr pollution eptsodes. [Carnow, B. W., Calif. Air Environment 4,7(1974).] 


\section{Nonradioactive Particulates}

Nodeling of Deposition in the Kespiratory Tract

Quantitative wodeling studies have been made of the fraction of particles deposiced in the various reglons of the respiritory tract. These reglons Include the alveolar region (for both nose and mouth breathing); the tracheobronchial tree (for botl: nose and mouth breathing); the oral cavity, pharynx, and larynx (for mouth breathing); the nasopherynx, pharynx, and larynx (for nuse breathing); the clliated nasal passages (for nose breathing); and the anterfor unciliated nares (for nose breathing). The various models differ somewhat in their quantitative predictions, depending on the assumptions made, but qualitatively their results are much the sane.

Three conclusions can be drawn about deposition in the alveolar region:

- There is a maximum efficlency of deposition at a particle size between 1 and $2 \mathrm{um}$.

- There is a mintmum efficiency at about $0.5 \mu \mathrm{m}$.

- The percentage of particle deposition for sizes less than $0.1 \mu \mathrm{m}$ is just as great as for sizes more than $1 \mu \mathrm{m}$.

These conclusions must be weighed by the particle size distribution for any particular case of intereat.

Figures 6 and 7 illugtrate the clearing mechanisms and particle termination points in the human body. Figure 8 represents the calculated effictencies of deposition of particles of various sizes, in the traceobronchlal and alveolar reglons of the resplratory system, and shows the size for minimum efficiency. A minimum in the removal effictency with respect to particle size artses in the transition range between removal by Brownian motion and by impaction. Figure 9 shows the deposition efficiencies.

\section{Epidemiological Effects}

At concentrations of $750 \mu \mathrm{g} / \mathrm{m}^{3}$ and higher for particulates on a $24-\mathrm{h}$ average, accompanied by sulfur dioxide concentrations of $715 \mathrm{\mu g} / \mathrm{m}^{3}$ and higher, excess deaths and a considerable Increase in Illness may occur. 
A decrease from 140 to $60 \mathrm{\mu g} / \mathrm{m}^{3}$ (annual mean) in particulate concentrations may be accompanied by a decrease In mean sputum volume in industrial workers.

If concentrat tons above $300 \mu \mathrm{g} / \mathrm{m}^{3}$ for particulates persist on a $24-\mathrm{h}$ Lverage and are accompanied by sulfur dioxide concentrations exceeding 630 $\mathrm{Hg} / \mathrm{m}^{3}$ over the same perlod, chrontc bronchttis pacients will likely suffer acute worsening of symptoms.

At concentracions over $200 \mathrm{\mu g} / \mathrm{m}^{3}$ for particulaces on a $24-\mathrm{h}$ average, accompanied by concentrations of tulfur dioxide exceeding $250 \mathrm{wg} / \mathrm{m}^{3}$ over the same average period, increased absence of Industrial woxkexs due to fliness may occur.

Where concentrations range from 100 to $130 \mathrm{\mu g} / \mathrm{at}^{3}$ and above for particulates (annual mean) with sulfur dloxide concentrarions (annual mean) greater than $120 \mu \mathrm{g} / \mathrm{m}^{3}$, children residing in such areas are 1 ikely to experience increased incidence of certain respiratory diseases.

At concentrations above $100 \mu \mathrm{g} / \mathrm{m}^{3}$ for particulates (annual geometric mean) with sulfation levels above $30 \mathrm{mg} / \mathrm{cm}^{2} \cdot$ mo, increased death $\mathrm{rates}$ for persons over 50 years of age are likely. These sulfarion levels correspond to $\mathrm{SO}_{2}$ concentrations of $120 \mathrm{\mu g}_{\mathrm{g}} \mathrm{m}^{3}$, or $0.04 \mathrm{ppm}$.

The EPA cites sulfates as being the sulfur oxide most suspected of causing adverse health effects. However, since the assumption that the health effects of sulfur-bearing afr emissions are largely due to the formation of sulfates is theoretical, further confirmation is needed and no air quality standard for sulfates has yet been established.

Table 6 relates infant and total mortality rates for 11.4 Standard Metropolitan Statistical Areas In the U.S. to air emissfons and other factors. As the biweekly minimum level of suspended particulates increases, the deach rate rises significantly. Moreover, the death rate increases with (1) the population density of the area, (2) the proportion of nonwhites, (3) the proportion of people over age 65, and (4) the proportion of poor families. 


\section{Toxirological and Phystological Ef fecto}

Particulate matcer may exert a coxic effect through one or mora of three mechanisus:

- The particle may be fntrinsically toxic due to its inherent chemlcal or physical properties.

- The particle may interfere with one or more of the clearance mechanisms in the respiratory tract.

- The particle may act as the carrier of an adsorbed toxfc substance.

Results of a study of asthmatics in the Salt Lake Basin showed the highest morbidity rates were associated with the highest suspended sulfate levels. However, the most consistently linked emission was total suspended particulates and the least impressive effect could be acributed to sulfur dioxide exposure (Fig. 20). The threshold for aggravation of astima by suspended sulfates was $1.4 \mu \mathrm{g} / \mathrm{m}^{3}$ on cooler days (30 to $50^{\circ} \mathrm{F}$ mintmuru ceoperacure).

In a study of cardiopulmonary patients in the New York metropolition area, the strongest and most consistent emission effects were found for suspended sulfates which were linked to a rorsening of symptoms, particularly shortness of breath, cough, and increased production of phlegm. The worsening of symptoms which was attributed to suspended sulfates always persisted af ter the correction for the effects attributable to cemperacure and other entssions.

A study of school children in Cincinnati found that the ventilatory function of children residing in an industrial valley of cincinnati was significantly lower than the performance of children living in a clean area of the metropolitan region. Differences in suspended sulfate levels were found to be most closely associated with differences in ventilatory performance.

Two significant tox 1 zological findings have resulced from the $f$ ew studies of sulfuric acid and sulfate particulates. First, sulfurlc acid and sulfate particulates are much more potent irritants than $\mathrm{SO}_{2}$ gas alone. Secondly, the mass weight of sulfates is an insufficient basis on which to predict irritant potency; particle size and chemical composition determine the toxic potential of sulfates (Tables 7-10). 
A highly variable dose-response relationship exists between $\mathrm{SO}_{2}$ and particulare levels and health impact on different populations (Fig. 11 and Tuble 11\}. In sumary, there is no thresiold for healtin effects. The outcome - health or disease, living or dying - depends upon the number of environmental stressors and the Intensity of each, matched against the resistance of the host and the host's adaptive ability.

Tive effects of ocher emissions, particularly cigarette smoking and heavy occupational exposure to Irritant gases, act synergistically with $\mathrm{SO}_{2}$ and particulates. The very large population already at risk because of such sthe? factors Increases the need for controlled levels of $\mathrm{SO}_{2}$ and particulates necessary to protect the health of these people:

of particular interest for coal-fired power plants, a synergistic potentlating ef fect has been observed between some particulates and sulfur dioxide. However, not all particulates potent late the response to sulfur dioxide, nor does not particulate potentlate the response of all irritant bases. Both the solubility of sulfur dioxide and its catalytic oxidation to sulfurle acid play a major role in its potent lation by certain particulates. The presence of carbon or soot as a common particulate is noteworthy, Inasmuch as carbon is an effictent adsorber of a wide range of organic and Inorganic compounds.

Few particulates commonly found in the atmosphere appear to be Intrinsically toxic; from the point of view of coal-fired power plants, the most important toxic aerosol is sulfur trloxide, either as the free ozide or hydrated as sulfuric acid. Recently, there has been increased concern about some less coumon toxtc particulates, including lead, beryllium, and asbestos.

\section{References - Nonradioactive Particulates}

Alr Quality Criteria for Particulate Matter, Department of Bealth, Education, and Welfare, National Air Pollution Control Administration, Washington, DC, Publication AP-49 (1969).

Amdur, M. 0. , and M. Corn, "The Irritant Potency of Zinc Aumonium Sulfate of Different Particle Sizes," Amer. Ind. Hyg. Assoc. J, 24, 326 (July-August 1963).

Carnow, B. W., Cal1f. A1r Environment 4, 7 (1974). 
Cost of Clean A1r, Annual Report of the Administrator of the EPA, October 1973, 93rd Congress, 12th Session, Doc. No. 93-40, USGPO, Washington, DC.

Health Consequences of Sulfur Oxide: A Report from CHESS, 1970-1971, Environmental Protection Agency, Research Trlangle Park, NC, PubI. EPA650/1-74-004 (1974).

Lawther, P. J., in Proceed Ings of the Confecence on Fuel and the Environment, November 26-29, 1973 (Institute of Fuel, London, 1973), Vo1. 2, p. 123.

Noran, J. P., private communication (1974).

Pierrard, J. M., "Part I, Particulate Matter, Oxides of Sulfur, and Sulfuric Acid," J. Alr Poll. Cont. Assoc. 19, 632 (1969).

Ra11, D. P., private communtcation (1973). 
Table 6. Regressions relating infant and total mortality rates for 114

Standard Metropolitan Statistical Areas in the U.S. to air emissions and other factors. Values in parentheses are the $t$ statistic. For means and standard deviations (S.D.) of the variables, see Footnote b.*

\begin{tabular}{|c|c|c|c|c|c|c|}
\hline \multirow[b]{2}{*}{ Category } & \multirow[b]{2}{*}{$k^{c}$} & \multirow[b]{2}{*}{$\begin{array}{c}\text { Alt } \\
\text { emission } \\
\text { (minimum) } \\
\text { concentrac lons }\end{array}$} & \multicolumn{4}{|c|}{ Soctoeconomic } \\
\hline & & & $P / \mathrm{B}^{2^{d}}$ & $\begin{array}{l}\text { Yon- } \\
\text { white } \\
(z)\end{array}$ & $\begin{array}{c}\text { Over } \\
65 \\
(z)\end{array}$ & $\begin{array}{c}\text { Poor } \\
(z)\end{array}$ \\
\hline \multicolumn{7}{|c|}{ Total death rate } \\
\hline 2.Particulates & .804 & $\begin{array}{c}0.102 \\
(2.83)\end{array}$ & $\begin{array}{r}0.001 \\
(2.58)\end{array}$ & $\begin{array}{r}0.032 \\
(3.41)\end{array}$ & $\begin{array}{r}0.682 \\
(18.37)\end{array}$ & $\begin{array}{r}0.013 \\
(0.93)\end{array}$ \\
\hline 2. Sulfates & .813 & $\begin{array}{c}0.025 \\
(3.73)\end{array}$ & $\begin{array}{r}0.001 \\
(1.86)\end{array}$ & $\begin{array}{r}0.033 \\
(3.56)\end{array}$ & $\begin{array}{r}0.652 \\
(17.60)\end{array}$ & $\begin{array}{r}0.006 \\
(0.49)\end{array}$ \\
\hline \multicolumn{7}{|c|}{ Death rate for Infants of less than 1 year } \\
\hline 3.Part1culates & .545 & $\begin{array}{c}0.393 \\
(3.07)\end{array}$ & & $\begin{array}{r}0.190 \\
(6.63)\end{array}$ & - & $\begin{array}{r}0.150 \\
(3.28)\end{array}$ \\
\hline 4. Sulfates & .522 & $\begin{array}{r}0.150 \\
(1.91)\end{array}$ & & $\begin{array}{r}0.200 \\
(6.83)\end{array}$ & & $\begin{array}{r}0.123 \\
(2.70)\end{array}$ \\
\hline & Death & rate Eor Infants & less thar & 28 days & old & \\
\hline 5.Particulates & .260 & $\begin{array}{r}0.273 \\
(2.48)\end{array}$ & & $\begin{array}{r}0.089 \\
(3.61)\end{array}$ & & $\begin{array}{r}0.063 \\
(1.60)\end{array}$ \\
\hline G.Sulfates & $.2 \dot{0} 3$ & $\begin{array}{r}0.170 \\
(2.57)\end{array}$ & & $\begin{array}{r}0.097 \\
(3.96)\end{array}$ & & $\begin{array}{r}0.047 \\
(1.23)\end{array}$ \\
\hline \multicolumn{7}{|c|}{ Fetal death rate } \\
\hline 7. Particulates & .434 & $\begin{array}{r}0.274 \\
(2.02)\end{array}$ & $\begin{array}{r}0.004 \\
(2.01)\end{array}$ & $\begin{array}{r}0.171 \\
(5.70)\end{array}$ & & $\begin{array}{r}0.106 \\
(2.11)\end{array}$ \\
\hline 8. Sulfates & .434 & $\begin{array}{r}0.171 \\
(1.95)\end{array}$ & $\begin{array}{r}0.004 \\
(1.82)\end{array}$ & $\begin{array}{r}0.181 \\
(5.87)\end{array}$ & & $\begin{array}{r}0.085 \\
(1.71)\end{array}$ \\
\hline
\end{tabular}

${ }^{a}$ The $t$ stacistic: for a one-talled t-test, a value of 1.65 Indicates significance at the .05 level.

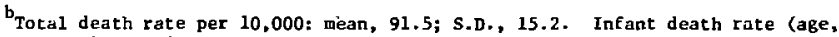
<1 year) per 10,000 1Ive births: mean, 255.1; S.D., 36.1. 1nfant death race (age, <28 days) per 10,000 live births: mean, 188.0; S.D., 24.4. Fetal death -ate per 10,000 live births: mean, 153.9; S.D., 34.4. Suspended particulates $\left(\mu \mathrm{g} / \mathrm{m}^{3}\right)$, ginimum reading for a biweekly perlod: mean, 45.2 ; S.D., 18.7. Total sulfates $\left(\mu_{\mathrm{g}} / \mathrm{m}^{3}\right)(\times 10)$, minimug reading for a biweekly perlod: mean, 46.9; S.D., 30.6. Persons per square m1le: mean, 763.4; S.D., 1387.9. Percentage of nonwhtes in population $(x 10)$ : mean, 125.2; S.D., 102.8. Percentage of population over $65(x 10)$ : mean, 84.2; S.D., 21.2 . Percentage of families with incomes under $\$ 3000(\times 10)$ : mean, 181.6; S.D., 65.7.

The coefficlent of determination: a value of .804 fadicates a multiple correlation coefficlent of .90 , and Indicates that 80 percent of the variation in the death rate is "explained" b; the regression.

$\mathrm{d}_{\text {Persons per aquake mile. }}$

*[Lave, L. B., and E. P. Seskin, Science 169, 725 (1970).] 
Table 7. Best jucigment estimates of exission thresholds for adverse effects of short-term exposures. *

\begin{tabular}{|c|c|c|c|}
\hline \multirow[b]{2}{*}{ Ef fect } & \multicolumn{3}{|c|}{ Threshold $\left(\mu \mathrm{g} / \mathrm{m}^{3}\right)$} \\
\hline & $\begin{array}{l}\text { Sulfur dioxide } \\
\qquad(365)^{a}\end{array}$ & $\begin{array}{l}\text { Total suspended } \\
\text { particulate } \\
(260)^{a}\end{array}$ & $\begin{array}{c}\text { Suspended } \\
\text { sulfates } \\
\text { (no standard) }^{a}\end{array}$ \\
\hline $\begin{array}{l}\text { Aggravation of } \\
\text { cardlopulmonary } \\
\text { symptoms in elderly }\end{array}$ & $>365$ & $80-100$ & $8-10$ \\
\hline Aggravation of asthma & $180-250$ & 70 & $8-10$ \\
\hline
\end{tabular}

National Primary Air Quality Standard.

"IHealth Consequences of Sulfur Oxides: A Report from CHESS, 1970-1971, Environmenta1 Protection Agency, Research Triangle Park, NC, Publ. EPA-650/ $1-74-004(1974)$. I 
Tablo 8. Best judgment estimates of emission thresholds for adverse effects of long-term exposures.*

\begin{tabular}{lccc}
\hline & \multicolumn{2}{c}{ Threshold, annual average $\left(\mu \mathrm{g} / \mathrm{m}^{3}\right)$} \\
\cline { 2 - 4 } Effect & $\begin{array}{c}\text { Sulfur dioxide } \\
(80)^{\mathrm{a}}\end{array}$ & $\begin{array}{c}\text { Total suspended } \\
\text { particulates } \\
(75)^{\mathrm{a}}\end{array}$ & $\begin{array}{c}\text { Suspended } \\
\text { sulfates } \\
\text { (no standard) }\end{array}$ \\
\hline $\begin{array}{l}\text { Increased prevalence of } \\
\text { chronic bronchitis } \\
\text { in adults }\end{array}$ & 95 & 100 & 15 \\
$\begin{array}{l}\text { Increased acute lower } \\
\text { respiratory disease } \\
\text { in children }\end{array}$ & 95 & 102 & 15 \\
$\begin{array}{l}\text { Increased frequency of } \\
\text { acute respiratory } \\
\text { disease in families }\end{array}$ & 106 & 151 & 13 \\
$\begin{array}{l}\text { Decreased lung function } \\
\text { of children }\end{array}$ & 200 & 100 & 15 \\
\hline
\end{tabular}

National Primary Atr Quality Standard. The particulate standard is a geometric mean; the equivalent arithmetic mean would be about $85 \mu \mathrm{g} / \mathrm{m}^{3}$.

* [Health Consequences of Sulfur Oxides: A Report from CHESS, 1970-1971, Environmental Protection Agency, Research Trłangle Park, NC, Publ. EPA-650f 1-74-004 (1974).] 
Table 9. Summary of CHESS studies relating long-term emission exposures to adve:se effects on human health.*

\begin{tabular}{|c|c|c|c|c|c|}
\hline \multirow[b]{2}{*}{ Adverse effect } & \multirow[b]{2}{*}{$\begin{array}{l}\text { Type of } \\
\text { est imate }\end{array}$} & \multirow[b]{2}{*}{$\begin{array}{l}\text { Duration } \\
\text { of } \\
\text { exposure } \\
(y)\end{array}$} & \multicolumn{3}{|c|}{$\begin{array}{c}\text { Annual average levels linked to adverse } \\
\text { health ef Eects }\left(\mu \mathrm{g} / \mathrm{m}^{3}\right)\end{array}$} \\
\hline & & & $\begin{array}{l}\text { Sulfur } \\
\text { dioxide } \\
(80)^{a}\end{array}$ & $\begin{array}{c}\text { Total } \\
\text { suspended } \\
\text { particulates } \\
(75)^{a}\end{array}$ & $\begin{array}{l}\text { Suspended } \\
\text { sulfates } \\
\text { (no standard) }\end{array}$ \\
\hline $\begin{array}{l}\text { Increase in prevalence of chronic } \\
\text { bronchitis in adults }\end{array}$ & $\begin{array}{l}\text { Worst case } \\
\text { Least case } \\
\text { Best Judgment }\end{array}$ & $\begin{array}{r}3 \\
10 \\
6\end{array}$ & $\begin{array}{r}62 \\
374 \\
95\end{array}$ & $\begin{array}{r}65 \\
179 \\
100\end{array}$ & $\begin{array}{l}12 \\
20 \\
15\end{array}$ \\
\hline $\begin{array}{l}\text { Increases in acute lower } \\
\text { respiratory tract Infections } \\
\text { in children }\end{array}$ & $\begin{array}{l}\text { Worst case } \\
\text { Least case } \\
\text { Best judgment }\end{array}$ & $\begin{array}{l}3 \\
3 \\
3\end{array}$ & $\begin{array}{r}92 \\
177 \\
95\end{array}$ & $\begin{array}{r}65 \\
102 \\
102\end{array}$ & $\begin{array}{l}7.2 \\
15 \\
15\end{array}$ \\
\hline $\begin{array}{l}\text { Increase in frequency or severity } \\
\text { of acute respiratory fllness } \\
\text { in families }\end{array}$ & $\begin{array}{l}\text { Worst case } \\
\text { Least case } \\
\text { Best Judgment }\end{array}$ & $\begin{array}{l}1 \\
3 \\
3\end{array}$ & $\begin{array}{r}50 \\
210 \\
106\end{array}$ & $\begin{array}{l}104 \\
159 \\
151\end{array}$ & $\begin{array}{l}14 \\
16 \\
15\end{array}$ \\
\hline $\begin{array}{l}\text { Subtle decreases in childhood } \\
\text { "entilatory function }\end{array}$ & $\begin{array}{l}\text { Worst case } \\
\text { Least case } \\
\text { Best judgment }\end{array}$ & $\begin{array}{r}1 \\
9 \\
8-9\end{array}$ & $\begin{array}{r}57 \\
435 \\
200\end{array}$ & $\begin{array}{r}96 \\
200 \\
100\end{array}$ & $\begin{array}{r}9 \\
28 \\
13\end{array}$ \\
\hline
\end{tabular}

a National Primary Ambient Air Quality Sţandard. The particulate standard is a geometric mean; the equivalent arithmetic mean would be about $85 \mathrm{\mu g} / \mathrm{m}^{3}$.

* [Health Consequences of Sulfur Oxides: A Report from CHESS, 1970-1971, Environmental Protectlon Agency, Regearch Triangle Park, NC, Pub1. EPA-650/1-74-004 (1974).] 
Table 10. Summary of threshold estimates for adverse effects of short-term exposure. *

\begin{tabular}{|c|c|c|c|c|c|}
\hline \multirow[b]{2}{*}{ Adverse effect } & \multirow[b]{2}{*}{$\begin{array}{l}\text { Type of } \\
\text { est imate }\end{array}$} & \multirow[b]{2}{*}{$\begin{array}{l}\text { HInfmum } \\
\text { temper- } \\
\left.\text { ature ( }{ }^{\circ} \mathrm{F}\right)\end{array}$} & \multicolumn{3}{|c|}{$\begin{array}{l}\text { Daily average levels linked to } \\
\text { adver }{ }^{2} \text { health effects }{ }^{a}\left(\mathrm{Hg} / \mathrm{a}^{3}\right)\end{array}$} \\
\hline & & & $\begin{array}{l}\text { Sulfur } \\
\text { dioxide } \\
(365)^{b}\end{array}$ & $\begin{array}{c}\text { Total } \\
\text { Buspended } \\
\text { particulate } \\
(260)^{b}\end{array}$ & $\begin{array}{c}\text { Suspended } \\
\text { sulfur } \\
\text { (no standard) }\end{array}$ \\
\hline \multicolumn{6}{|l|}{$\begin{array}{l}\text { Aggravation of } \\
\text { cardiopulmonary } \\
\text { sympeoms in } \\
\text { elderly }\end{array}$} \\
\hline \multirow[t]{2}{*}{ "We11" panel } & $\begin{array}{l}\text { Worgt case } \\
\text { Least case } \\
\text { Best Judgment }\end{array}$ & $20-40$ & $\begin{array}{l}81-365 \\
\text { AE } \\
\text { NPE }\end{array}$ & $\begin{array}{l}\text { NPE } \\
\text { NE } \\
\text { NPE }\end{array}$ & $\begin{array}{l}<1 \\
\mathrm{NE} \\
8-10\end{array}$ \\
\hline & $\begin{array}{l}\text { Worst case } \\
\text { Least case } \\
\text { Best Judgrent }\end{array}$ & $>40$ & $\begin{array}{l}81-365 \\
\text { NE } \\
\text { NPE }\end{array}$ & $\begin{array}{l}68 \\
\mathrm{NE} \\
80-100\end{array}$ & $\begin{array}{l}2 \\
10 \\
8-10\end{array}$ \\
\hline "Heart" panel & $\begin{array}{l}\text { Worst case } \\
\text { Least case } \\
\text { Best Judgment }\end{array}$ & $>40$ & $\begin{array}{l}\text { NPE } \\
\text { NE } \\
\text { NPE }\end{array}$ & $\begin{array}{l}76-260 \\
\text { NE } \\
\text { NPE }\end{array}$ & $\begin{array}{l}10 \\
10-20 \\
10\end{array}$ \\
\hline \multirow[t]{2}{*}{ "Iung" panel } & $\begin{array}{l}\text { Worst case } \\
\text { Least case } \\
\text { Best judgment }\end{array}$ & $20-40$ & $\begin{array}{l}\text { NPE } \\
\text { NE } \\
\text { NPE }\end{array}$ & $\begin{array}{l}76-260 \\
\text { NE } \\
\text { NPE }\end{array}$ & $\begin{array}{l}6 \\
\mathrm{NE} \\
10\end{array}$ \\
\hline & $\begin{array}{l}\text { Worst case } \\
\text { Least case } \\
\text { Best judgment }\end{array}$ & $>40$ & $\begin{array}{l}\text { NPE } \\
\text { NE } \\
\text { NPE }\end{array}$ & $\begin{array}{l}76-260 \\
\text { NE } \\
\text { NPE }\end{array}$ & $\begin{array}{l}11 \\
12 \\
12\end{array}$ \\
\hline \multirow[t]{2}{*}{$\begin{array}{l}\text { "Heact and lung" } \\
\text { panel }\end{array}$} & $\begin{array}{l}\text { Worst case } \\
\text { Least case } \\
\text { Best judgment }\end{array}$ & $20-40$ & $\begin{array}{l}181 \\
\mathrm{NE} \\
\mathrm{NPE}\end{array}$ & $\begin{array}{l}47 \\
\mathrm{NE} \\
80-100\end{array}$ & $\begin{array}{l}9 \\
10 \\
10\end{array}$ \\
\hline & $\begin{array}{l}\text { Worst case } \\
\text { Least case } \\
\text { Best fudgment }\end{array}$ & $>40$ & $\begin{array}{l}\text { NPE } \\
\text { NE } \\
\text { NPE }\end{array}$ & $\begin{array}{l}76 \\
\text { NE } \\
\text { NPE }\end{array}$ & $\begin{array}{l}6 \\
17 \\
10\end{array}$ \\
\hline \multirow[t]{2}{*}{$\begin{array}{l}\text { Aggravation of } \\
\text { eathma }\end{array}$} & $\begin{array}{l}\text { Worst case } \\
\text { Least case } \\
\text { Best fudgment }\end{array}$ & $30-50$ & $\begin{array}{l}\text { NPE } \\
\text { NE } \\
\text { NPE }\end{array}$ & $\begin{array}{l}61-75 \\
N E \\
105\end{array}$ & $\begin{array}{l}8 \\
\text { NE } \\
9-10\end{array}$ \\
\hline & $\begin{array}{l}\text { Worst case } \\
\text { Least case } \\
\text { Best Judgment }\end{array}$ & $>50$ & $\begin{array}{l}23 \\
\mathrm{NE} \\
180-250^{\mathrm{c}}\end{array}$ & $\begin{array}{l}61-75 \\
\mathrm{NE} \\
70\end{array}$ & $\begin{array}{l}<] \\
10 \\
8\end{array}$ \\
\hline
\end{tabular}

aE: no effect below primary standard, or simply no effect for suspended sulfates, fo which no primary standard has been established. NPE: no proven effect below prinary standard, or simply no proven effect for suspended sulfates.

bational Primary AIr Quality Standard.

Chis fudgment is based on presently sumpartzed studies and on a previously reported ChESS study of aathme in New Cumberland, West Virginia.

* [Health Consequences of Sulfur Oxides: A Report from CHESS, 1970-1971, Environmen Protection Agency, Research Trlangle Park, NC, Pub1. EPA-650/1-74-004 (1974).] 
Table 11. Summary of dose/response relationships for effects of particles and $\mathrm{SO}_{2}$ and health (old results, leading to original standards).*

\begin{tabular}{|c|c|c|c|c|}
\hline \multirow{2}{*}{$\begin{array}{l}\text { Averaging time } \\
\text { for } \\
\text { measurements }\end{array}$} & \multicolumn{4}{|c|}{ Approximate concentration levels } \\
\hline & Place & $\begin{array}{l}\text { Part Icles } \\
\left(\mu_{\mathrm{g}} / \mathrm{m}^{3}\right)\end{array}$ & $\mathrm{SO}_{\mathrm{Z}_{3}}$ & Effect \\
\hline $24 \mathrm{~h}$ & London & 2000 & 1144 & Mortality \\
\hline $24 \mathrm{~b}$ & London & 750 & 700 & Mortality \\
\hline $24 \mathrm{~h}$ & London & 300 & 600 & Deterioration of paticnts \\
\hline Week1y mean & London & 200 & 400 & $\begin{array}{l}\text { Prevalence or incidence of } \\
\text { respiratory illnesses }\end{array}$ \\
\hline $24 \mathrm{~h}$ & New York & $6^{a}$ & 1500 & Mortality \\
\hline Winter mean & Britain & $100-200$ & $100-200$ & $\begin{array}{l}\text { Incapacity for work from } \\
\text { bronchitis }\end{array}$ \\
\hline \multirow[t]{6}{*}{ Annual } & Britain & 70 & 90 & $\begin{array}{l}\text { Lower respiratoiy infec- } \\
\text { tions in children }\end{array}$ \\
\hline & Britain & 100 & 100 & $\begin{array}{l}\text { Upper and lower respira- } \\
\text { tory infections in } \\
\text { children }\end{array}$ \\
\hline & Britain & 100 & 100 & Bronchitis prevalencs \\
\hline & Britain & 100 & 100 & Prevalence of $s /$ mptoms \\
\hline & Buffalo & 100 & $0.30^{b}$ & Respiratory jortality \\
\hline & Berlin, NH & 180 & $0.73^{b}$ & $\begin{array}{l}\text { Increased respiratory } \\
\text { symptoms } \\
\text { Decreased pulmonary } \\
\text { function }\end{array}$ \\
\hline
\end{tabular}

${ }^{\mathrm{a}}$ COHS.

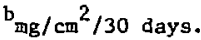

* Health Consequences of Sulfur OxIdes: A Report from CHESS, 1970-1971, Environmental Protection Agency, Research Triangle Park, NC, Pub1. EPA-650/1-74-004 (1974).] 
1. Out with expired oir.

2. Caught in nose, our with mueus.
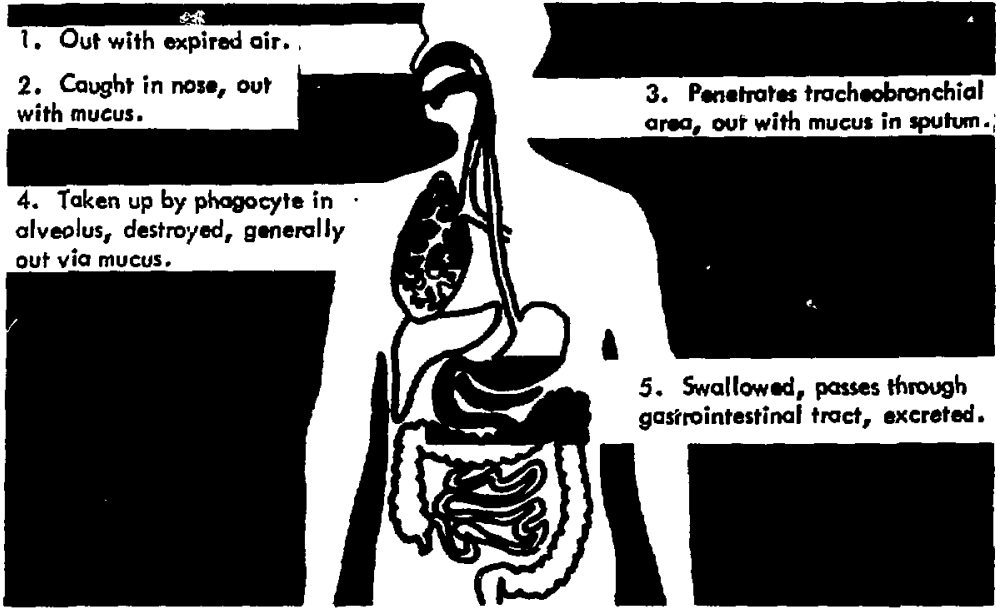
area, out with mucus in sputum.

4. Taken up by phogocyle in alveolus, destroyed, generally out via mucus.

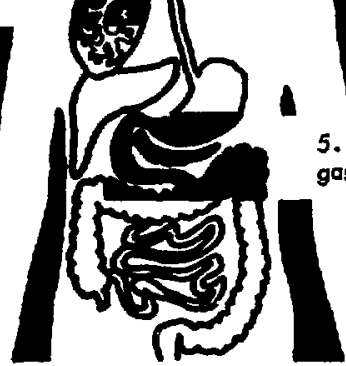

e.

5. Swallowed, passes through gasirointestinal tract, excreted.

Fig. 6. Particle clearing mechanisms in the human body. The body ${ }^{t} s$ defenses against invasion by foreign particles are considerable, but can be overwhelmed by excessive amounts of particulate emissions, by breathing particles in combination with gases, or by particles that are infectlous, 1rritacing, or toxic. [Brodine, V., Air Pollution (Harcourt, Brace, and Jovanovich, New York, 1973), p. 122.] 


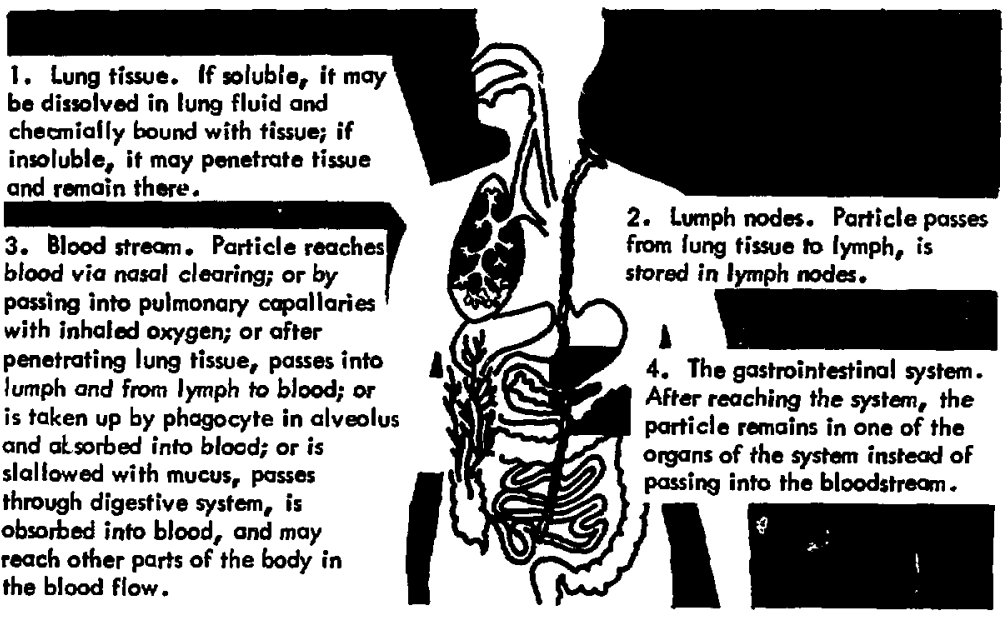

FIg. 7. Termination points of particle not cleared by one of the particle clearing mechanisms in the human body. [Brodine, V., Air Pollucton (Harcourt, Brace, and Jovanovich, New York, 1973), p. 123.] 


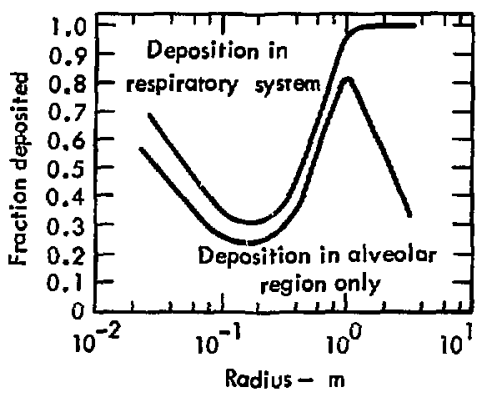

Fig. 8. Calculated fraction of particles deposited in the resplratory cract as a function of particle radius. [From Alr Qualicy Crfterta for Particulate Matter, Department of Health, Education, and Welfare, National Atr Pollution Control Administration, Washington, DC, Publ. AP-49 (1969), P. 114, and Ffnietsen, W., Arch. Ges. Physial. 236, 367 (1935).]

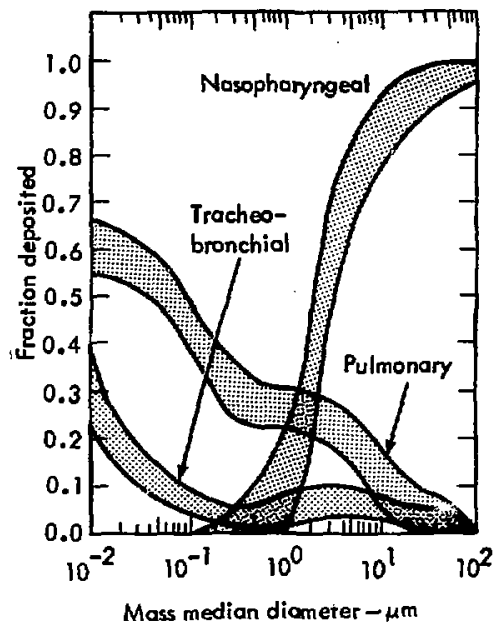

Fig. 9. Fraction of parcicles deposited in the three respiracory cract compartmencs as a function of parcicle diamecer. [AIr Quality Criteria for PartIculate Matter, Department of Health, Education, and Welfare, National Afr Pollution Control Administration, Washington, DC, Publ. AP-49 (1969). p.115.] 


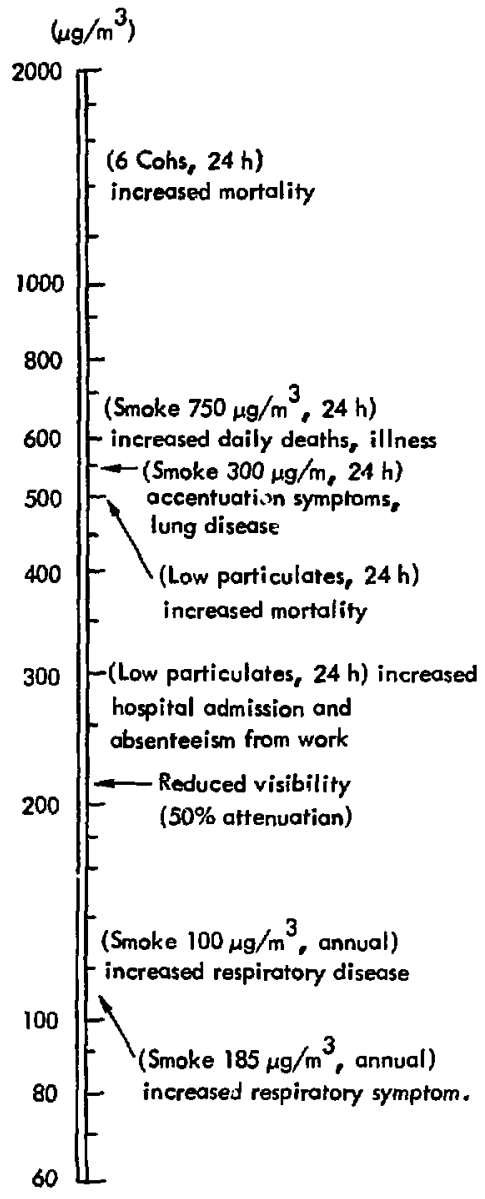

Fig. 10. Alr quality criteria for particulates. $26 \mu \mathrm{g} / \mathrm{m}^{3} \mathrm{SO}_{2}$ $=0.01$ ppm (vol). [Lawrence Livermore Laboratory, staff compilation.] 

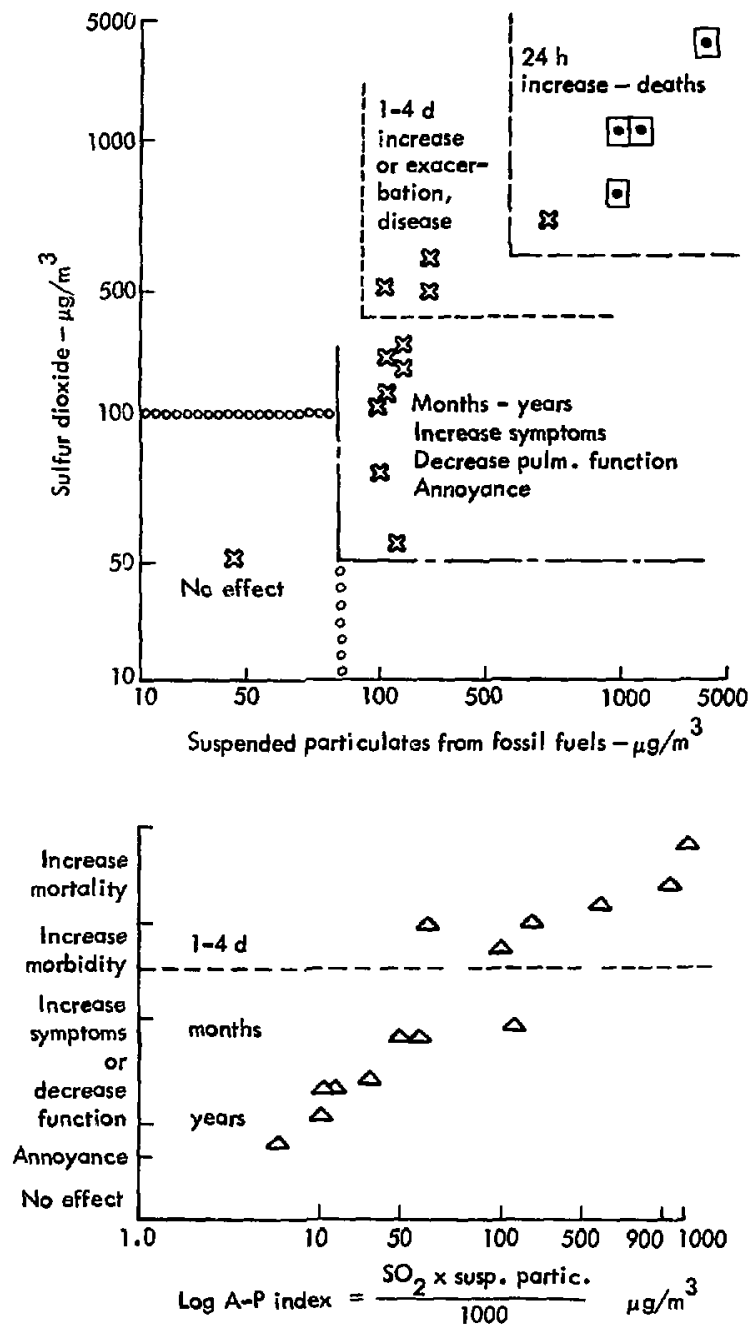

Fig. 11. Dose response curve for sulfur dioxide and total suspended particulates. [Ferris, B., personal communication (September 1973).] 
The principal problems are nut creaced by the hydrocarbon directly, but by compounds derived from the atmospheric reactions of hydrocarbons and their derlvatives with other substances (Tables 12-15).

Reactive hydrocarbons become involved in photochemical air emissions not because of their exposure to gunlight, but because of their reactions with atomic oxygen, electronically excited $\mathrm{O}_{2}, \mathrm{O}_{3}$, and free radicals generated by the action of sunlight on other components in the atmosphere, most particularly $\mathrm{NO}_{2}$

The photochemical reactions have the net effect of producing photochemical oxidants (sometimes reported as "ozone"). These are the compounds that in association with $\mathrm{NO}_{2}$ and particulate matter produce the recognizable smog-type air pollution chac reduces visibility, colors the atmosphere, irritates the eyes and respiratory system, and is toxic to flora and fauna.

Certain hydrocarbons - In particular, aromatics with three to six bepzene rings - constitute the most important group of known or suspected carcinogens, and potentially harmful effects may result from repeating exposure. The more rexctive ingredients, such as echylene, disappear quite rapidly (within 24 h or less), while the less reactive components may have iffetimes measured in weeks or months.

\section{References - Organic Gases}

Afr Quality Criterla for Photochemical Oxidants, Department of Health, Education and Welfare, Nacional Alr Pollution Control Adminiscration, Washington, DC, Publication AP-63 (1970).

American Assoctation for the Advancement of Sclence, Air Conservation, The Report of the Air Conservation Commission, No. 80, Washington, DC (1965).

Haagen-Smit, A., and L. G. Wayne, "Atmospheric Reactions and Scavenging Processes," In Alr Pollucion, Vol. 1; Alr Pollution and Irs Effects, 2d edition, A. C. Stern, Ed. (Academic Press, New York, 1968).

Nationwide Inventory of Alr Pollutant Emissions, 1968. Public Health Service, Eqvironmental Health Service, Raleigh, North Carolina (1970), p. 12.

Pikul, R. P., and R. Rabin, Program Plan for the Environmental Effects of Energy, Mitre Corp., HcLeon, VA, Publ. MTR-6726 (1974).

Shuck, E. A., and E. R. Stephens, "Oxides of Nitrogen," In Advances in Environmental Sciences, J. Pitts and R. Metcalf, Fds. (Wiley-Interscience, New York, 1969), Vol. 1 . 
Table 12. Toxicity of saturated aliphatic hydrocarbons (through octane).

\begin{tabular}{|c|c|c|c|c|c|c|c|c|c|c|c|}
\hline \multirow[b]{2}{*}{ Hydrocarbon } & \multicolumn{11}{|c|}{ Concentration (ppri) } \\
\hline & 100.000 & 50,000 & 40,000 & 30,000 & 20,000 & 15,000 & 10,000 & 5,000 & 2.000 & 1.000 & 500 \\
\hline $\begin{array}{l}\text { Methane } \\
\mathrm{CH}_{4}\end{array}$ & No effect & & & & & & & & & & \\
\hline $\begin{array}{l}\text { Ethane } \\
\mathrm{C}_{2} \mathrm{H}_{6}\end{array}$ & & $\begin{array}{l}\text { No } \\
\text { ef } \mathrm{fect}\end{array}$ & & & & & & & & & \\
\hline $\begin{array}{c}\text { Propane } \\
\mathrm{C}_{3} \mathrm{H}_{8}\end{array}$ & $\begin{array}{l}\text { No frritation } \\
\text { noticed } \\
\text { Dizziness } \\
\text { in a few } \\
\text { min }\end{array}$ & & & & $\begin{array}{l}\text { Odor } \\
\text { not de- } \\
\text { lect ed }\end{array}$ & & $\begin{array}{l}\text { No symp- } \\
\text { tams } \\
\text { after } \\
\text { brief ex- } \\
\text { posure }\end{array}$ & & & & \\
\hline $\begin{array}{l}\text { Butane } \\
\mathrm{C}_{4}^{13} 10\end{array}$ & & & & & & & $\begin{array}{l}\text { Drawsi- } \\
\text { ness in } \\
10 \text { mini }\end{array}$ & $\begin{array}{l}\text { Odor nut de- } \\
\text { lertable }\end{array}$ & & & \\
\hline $\begin{array}{r}\text { Pentane } \\
\mathrm{C}_{5}{ }^{\mathrm{H}} 12\end{array}$ & $\begin{array}{l}\text { Narcosis in } \\
5-60 \mathrm{~min}\end{array}$ & & & & & & & $\begin{array}{l}\text { Odor readjly } \\
\text { detectable; } \\
\text { No irrtiation or } \\
\text { symptoms in } \\
\text { lo min }\end{array}$ & & Tt, & \\
\hline $\begin{array}{l}\text { Hexane } \\
\mathrm{C}_{6} \mathrm{H}_{14}\end{array}$ & & & $\begin{array}{l}\text { Convul- } \\
\text { stons } \\
\text { ind } \\
\text { det it b }\end{array}$ & Warcosís & & & & $\begin{array}{l}\text { otzalness, } \\
\text { gidd iness, } \\
\text { in } 10 \mathrm{~m} h \mathrm{a}\end{array}$ & $\begin{array}{l}\text { No symp- } \\
\text { loms in } \\
10 \mathrm{~min}\end{array}$ & & $\tau L U$ \\
\hline $\begin{array}{r}\text { Heptane } \\
\mathrm{C}_{7} \mathrm{H}_{16}\end{array}$ & & & & & $\begin{array}{l}\text { Conval- } \\
\text { sians, } \\
\text { deatis } \\
\text { fo } 30- \\
60 \text { min }\end{array}$ & $\begin{array}{l}\text { Narensis } \\
\text { ln } 30- \\
60 \mathrm{~min}\end{array}$ & & 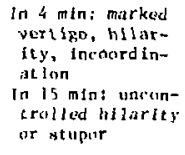 & $\begin{array}{l}\text { slight } \\
\text { verelgu } \\
\text { in } 4 \text { min }\end{array}$ & & $\mathrm{TLV}$ \\
\hline $\begin{array}{c}\text { Octane } \\
\mathrm{C}_{8} \mathrm{H}_{\mathrm{I}} \mathrm{B}\end{array}$ & & & & & & & $\begin{array}{l}\text { Narcosis } \\
\text { In } 30- \\
90 \mathrm{~min}\end{array}$ & & & & $72 . \mathrm{N}$ \\
\hline
\end{tabular}


Table 13. Comparative effects of acute and chrontc exposure to aromactc hydrocarbon vapors in a1r.*

\begin{tabular}{|c|c|c|c|c|c|c|}
\hline \multirow[b]{2}{*}{ Compound } & \multirow[b]{2}{*}{ Subject } & \multicolumn{2}{|c|}{ Concentration } & \multirow{2}{*}{$\begin{array}{l}\text { Daily } \\
\text { exposure } \\
\text { (h) }\end{array}$} & \multirow{2}{*}{$\begin{array}{l}\text { Exposure } \\
\text { (days) }\end{array}$} & \multirow[b]{2}{*}{ Effect } \\
\hline & & ppm & $\mathrm{mg} / \mathrm{m}^{3}$ & & & \\
\hline \multirow[t]{11}{*}{ Benzene } & Man & 25 & 80 & Acute & -- & Threshold limit value (TLV) \\
\hline & Man & 100 & 319 & Acute & -- & Nucous membrane irritition \\
\hline & Mice & 370 & 1,180 & Acute & -- & $\begin{array}{l}\text { Threshold for affecting the } \\
\text { central nervous system }\end{array}$ \\
\hline & Man & 3,000 & 9,570 & Acuce & -- & Endurable Eor $1 / 2-1$ i \\
\hline & Mice & 4,700 & 14,993 & Acule & -- & Prostration \\
\hline & Mice & 7,400 & 23,606 & Acute & -- & $\mathrm{LC}_{50}$ \\
\hline & Man & 7,500 & 23,925 & Acute & -- & Dangerous afcer $1 / 2-1$ h \\
\hline & Mice & 14,100 & 44,979 & Acute & -- & LC $_{100}$ \\
\hline & Rats & 17,800 & 56,782 & Acute & -- & $L C_{100}$ \\
\hline & Man & 20,000 & 63,800 & Acute & -- & Fatal after $5-10 \mathrm{~min}$ \\
\hline & Man & $50-100$ & $188-377$ & Acute & -- & No effect \\
\hline \multirow[t]{9}{*}{ Toluenc } & Man & 200 & 753 & 8 & 1 & $\begin{array}{l}\text { Mild fat goue, weakness, } \\
\text { confuston, skin partchesias }\end{array}$ \\
\hline & Man & 300 & 1,130 & 8 & 1 & Symptoms more pronounced \\
\hline & Man & 400 & 1,506 & 8 & 1 & Also: mental contusion \\
\hline & Man & $60 \mathrm{~s}$ & 2,259 & 3 & 1 & $\begin{array}{l}\text { Also: nausea, headache, } \\
\text { dizziness }\end{array}$ \\
\hline & Mar. & 600 & 2,259 & 8 & 1 & $\begin{array}{l}\text { Also: loss of coordination, } \\
\text { staggering gait. pupils } \\
\text { dilated }\end{array}$ \\
\hline & Mice & 2,700 & 10,166 & Acute & -- & Prostration \\
\hline & Mice & 6,700 & 25,226 & Acute & -- & $\mathrm{LC}_{50}$ \\
\hline & Mice & 9,500 & 35,768 & Acute & -- & {$\left[C_{\ln 0}\right.$} \\
\hline & Rat & 13,500 & 50,828 & Acute & -- & $L C_{1.00}$ \\
\hline \multirow[t]{7}{*}{ Styr eue } & $\begin{array}{l}\text { Man } \\
\text { Guinea }\end{array}$ & $\begin{array}{l}100 \\
650\end{array}$ & $\begin{array}{r}418 \\
2,714\end{array}$ & $\begin{array}{l}\text { Acute } \\
8\end{array}$ & 180 & $\begin{array}{l}\text { Threshold limit value (TLV) } \\
\text { No effect }\end{array}$ \\
\hline & Rat & 1,300 & 5,428 & 8 & 180 & Eye \& nasal irritation only \\
\hline & Rabbit & 1,300 & 5,428 & 8 & 180 & Eye \& nasal irritation only \\
\hline & $\begin{array}{l}\text { Guinea } \\
\text { pis }\end{array}$ & 1,300 & 5,428 & 8 & 180 & $10 \%$ deaths \\
\hline & $\begin{array}{c}\text { Guinea } \\
\text { pig \& } \\
\text { rat }\end{array}$ & 2,500 & 10,438 & 8 & 1 & $\begin{array}{l}\text { Some fatalficies; varying } \\
\text { degree of weaknass, } \\
\text { stupor, incoordination, } \\
\text { rremor, unconsciousness } \\
\text { (In } 10 \mathrm{~h} \text { ) }\end{array}$ \\
\hline & $\begin{array}{l}\text { Guinea } \\
\text { p1g \& } \\
\text { rac }\end{array}$ & 5,000 & 20,875 & $\mathbf{1}$ & 1 & Unconsciousness in $1 \mathrm{~h}$ \\
\hline & $\begin{array}{l}\text { Gufnea } \\
\text { pig } \\
\text { rat }\end{array}$ & 10,000 & 41,750 & $1 / 2-1$ & 1 & $\begin{array}{l}\text { Unconsciousness in } 10 \mathrm{mIn} \\
\text { deaths in } 30-60 \mathrm{~min}\end{array}$ \\
\hline \multirow[t]{3}{*}{ Xylene } & Mice & 174 & 755 & Acute & - & $\begin{array}{l}\text { Threshold for af fecting } \\
\text { central nervous system }\end{array}$ \\
\hline & Man & 200 & 868 & Acure & & Threshold limit value (TLV) \\
\hline & $\begin{array}{l}\text { Rat } \delta \\
\text { rabbit }\end{array}$ & 690 & 2,995 & 8 & 130 & No hematological ef fects \\
\hline
\end{tabular}

* Alir Quality Criterla fo" Hydrocarbons, Department of Health, Education, and Welfare, Nat Ional Air Pollution Control Association, Washington, DC, Publ. AP-64 (1970).] 
Table i4. Toxicity of unsaturated aliphatic hydrocarbons.*

\begin{tabular}{|c|c|c|c|c|c|c|c|c|c|c|c|c|}
\hline Hydrocarbon & 350,000 & 30,000 & 200,000 & 100,000 & 50,000 & 40,000 & 20.000 & 8,000 & 5,500 & 5,000 & 4,000 & 1,000 \\
\hline
\end{tabular}

Echylene

Propylene

1-butene

\section{1,3-butadiene}

2-methy11-1,3-

butadiene
MP1.

MPL TIN

MPL

NPL.

\begin{tabular}{|c|c|c|c|c|}
\hline Acetylene & $\begin{array}{l}\text { Uncon- } \\
\text { scious- } \\
\text { ness in } \\
5 \mathrm{~min}\end{array}$ & $\begin{array}{l}\text { Inco- } \\
\text { ordina- } \\
\text { tion }\end{array}$ & $\begin{array}{l}\text { Marked } \\
\text { intoxi- } \\
\text { cat ton }\end{array}$ & $\begin{array}{l}\text { Slight in- } \\
\text { toxicat- } \\
\text { ing } \\
\text { effect } \\
\text { on man }\end{array}$ \\
\hline
\end{tabular}
Irrication
of teye and upper $r:-$
spiratory
tract in
man

* [Alr Quality Criteria for Hydrccarbons, Department of Health, Education, and Welfare, National Alr Pollution Control Assoctation, Washington, DC, Publ. AP-64 (1970).] 
Table 15. Toxicological comparison of methanol and gasoline.*

Chemical nature

Threshold limit value

(TLV) and maximum

allowable concentration

(MAC)

Acute inhalation

Chronic inhalation

Aspiration inco lungs

Ingesticn

Skin contact

Warning odor
C.4 ${ }_{3} \mathrm{OH}$

200 ppm

Slight hazard

Moderate hazard

Slight hazard

Moderate hazard

Both defatting and irritating

Absorbed

through skin

Inadequate
Mixture of $c_{4}-c_{12}$ aliphatics plus aromatics

$500 \mathrm{ppm}$

$25 \mathrm{ppm}$ benzene
Slight hazard

Slight hazard

Very toxic

slight hazard

Little absorbed

Adequate

* [Berger, B. J., Environmental Aspects of Methanol as Vehicular Fuel: Health and Environmental Effects, Lawrence Livermore Laboratory, Rept. UCRL-76076 $(1974) \cdot 1$ 
Table 16 lists the major energy sources of trace metals in the air and their effects on human health.

\section{Beryllium}

Beryllium produces a varlety of effects depending upon the dose and the duration of exposure. A prominent lesion caused by beryllium is a granulomatous nodule found in the skin, subcutaneous tissues, and lungs. The most extensive changes are usually found in the lungs.

At present there is no evidence that communicy or industrial. exposure to beryllium compounds is associated with an increase in the Incidence of carcinoma in humans. The pathogenesis of health effects produced by beryllium is not clear.

\section{Cadmium}

Cadmium is toxic to man and to other living things in virtually all of its chemical forms. Although we do not know the thr ashold values at which chronlc effects begin to be noticeable, there is little indication that cadmium at any level serves any useful function in living organisms. Cadmium accumulates In the body, especially in the kidney and the liver, over many years.

Cadmium does not degrade in the environment, but accumulates in soils and sediments where it can enter various food chains. Such accumulations resulting from mining operations caused 1tai-1tal disease in Japan through the contamination of food and water.

Cadmium In the air is absorbed from the lungs and deposited in the kidney, liver, and arteries. It can produce destructive kidney changes, and liver damage has been caused by chrontc exposure to large amounts. Rats exposed for a lifetime to small doses of cadmium in drinking water exhibited arterial hypertension and the pathologlcal counterpart of the human disease.

Cadmium Is not Important as a constituent of coal, but it reacts with sulfhydryl enzymes such as the metals mercury and lead that are present in coal. It is Important to consider that the metals in coal have the same mode of action as other metals that enter the atmosphere from other sources, 
and these combinations of metals from different sources have the potential for causing additive toxicity.

We do not know the extent to which present environmental levels of cadmium in the U. $S$, are a health hazard, although some studies have implicaced cadmium in connection with hypertension and increased mortality. We do know that some $4 \%$ of surface waters sampled by the USGS in 1970 exceeded the Public Health Service drinking water standard of $0.01 \mathrm{ppm}$. Furthermore, the Bureau of Mines projects a two- to threefold increased demand for cadmium by the year 2000 .

\section{Mercury}

The symptoms of chronic mercury poisoning may appear after a few weeks of exposure or they may be delayed such longer. The characteristic sertous effects are psychic and emotional disturbances, loss of memory, tremors, and renal disease.

In considering the hazards of mercury, it is important to recagnize that its principal blochemical action is combined with sulfhydryl groups of Important enzymes. In this respect, mercury resembles arsenic, lead, and cadmium.

Known as a poison to industrial workers since ancient times, primarily as the metal or in simple tnorganic form, it has only recently been recognized as a lethal materfal in the environment In the form of salts of the alkyl compounds. These alkyl salts may be formed from fnorganic mercurials directly by chemical converston or by the action of certain microorganisms living in soils or sediments urider fresh-water bodies.

The widespread occurrence of methylmercury, the most toxlc form of mercury in man's fooc chain, catalyzes a continuing need to understand its effects. One of the major problems is the definttion of "safe" Ievels. Many of the answers needed to define "safe" levels will come from the study of humans exposed to Inordinately high levels.

\section{Nickel}

Nickel as nickel carbonyl causes cancer in animals and humans. Cancer of the respiratory tract has been inducid in rals by exposing them to nickel carbonyl. Nickel refinery workers are prone to develop lung cancer when exposed to nickel dust and fumes or nickel carbony1 for many years. Death 
rates from these causes are 2 to 25 times the rates expected in the general population. Nickel carbonyl is readily picked up from the lung by the blood although some nickel combines with lung proteins.

It is not known whether airborne nickel contains the carbonyl form, but the conditions are right for forming it in some power-plant comburtion equipment. It is formed when hot carbon monoxide passes over nickel.

Because of the carcinogeniclty of nickel in appropriate doses, it represents a potentlal hazard to urban dwellers, and the sources of atmospheric nickel should be decreased as fast as possible.

$\underline{\text { Lead }}$

Lead is an element with no known beneficlal function in human metabolism. It has been known for 2000 years that it is toxic; but in spite of Its recognition as a poison it is widely user in modern technology.

Acute toxicity results from excessive exposure, primarily from industrlal hygiene problems among occupationally exposed adults and from the pediatric lead polsoning associated largely with lead-iased paints.

To date, no cases of acute plumbism have been reported in adults with blood-lead below $80 \mu \mathrm{g} / 100 \mathrm{cc}$. Some authorlties believe that blological changes are exhibited at all lower exposures, that any change is undesirable, and that no threshold exists below which no damage results.

Laad is excreted from the body at nearly the same rate as introduced, the balance accumulating slowly in the body with age, princlpally in the bones, aorta, liver, kldney, pancreas, trachea, larynx, lungs, and skin. The bones on the average contain $91 \%$ of the total lead in the body. As lead Impairs the production of hemoglobin, it also causes oxygen starvation and anemia.

Lead has been observed to cause deleterlous effects in the liver, kidney, brain, and nervous system. These effects are assoctaced with blood lead levels in excess of $100 \mu \mathrm{g} / 100 \mathrm{ml}$ of blood in acute encephalopathy and $80 \mu \mathrm{g} / 100 \mathrm{mI}$ of blood in chronic encephalopathy.

Persons most prone to lead poisoning are workers occupationally exposed and children. Occupations Inc'ude Industrial lead-processing operations and employment near high traffic densities. Children exhibiting symptoms of lead toxicity have been observed eating chips of old paint and putty. 
Other factors such as urban atmospheric concentrations, ingestion of street dirt, ethnic diet, and ethnic and cultural factors have not been adequately evaluated. It is a curious fact that about 10 times as many cases of pediatric lead poisoning occur in midsummer, August, as occur in tine pertod from January through March.

Conclusive evidence is lacking that lead is emitted in auto exhaust in concentrations threatening human health, or that it has built up in the environment to such concentrations. Further, unt 11 very recently, no compre-hensive systems approach to the distribution and environmental effects of lead had been made, particularly at the ecosystem level.

\section{Other Metals}

Numerous other metals are present in coal, and they are emitted into the atmosphere at various concentrations. Most of them will interfere with physiological processes at approprlate concentrations. Thus, bismuth, antimony, tin, aluminum, barfum, strontium, zirconium, niobium, and vanadium are all present in coal and are released into the atmosphere by the burning of caal.

A number of metals that are essential for man are also found in the alr, but the intake of these metals is usually below $1 \%$ of the amounts in food and water.

The available information indicates that most of the metals present in the atmosphere do not represent a hazard to human health, but a few are present at levels that are near those known to produce adverse effects.

The estimation of the safety of various levels of metals in the air usually has to be based on experimental work done to establish safe levels for industrial exposure. The permissible levels are based on daily 8-h exposures rather than to the $24-\mathrm{h}$ exposures that the general population receives from alr emissions. Furthermore, the permissible industrial levels usually do not consider possible additlve or synergistic effects from combinations of metals which are always present in the atmosphere.

It is clear from the information avallable at the present time, even though it is incomplete, that reasonable efforts should be made to reduce the emission of metals and other toxicants from coal and other sources. 


\section{References - Trace Metals}

Boulding, R., "What is Pure Coa1?", Environment 18, 16 (January/February 1976). Buhler, D. R., Heavy Mecals in the Environment (University of Oregon, Eugene, OR, 1973).

Dawson, G. W., The Chemical Toxicity of Elements, Battelle Pacific Northwest Laboratories, Rechland, WA, Publ. BNWJ,-1B15 (1974).

Goldwater, L. J., "Mercury in the Environment," Sci. Amer. 224, 15 (1971).

Kle1n, D. H., "Trace Element Discharge from Coal Combustion for Power Production," Water Air Soll Pollution 5, 71 (1975).

Koakinen, J. W., et al., "Trace Element Behavior In Coal-Fired Power Plant," Env. Sct. Tech. 9, 862 (1975).

Grant, N., "Mercury in Man," Environment 13, 2 (1971).

Moffitt, A. E., Jr., and R. E. Kupel, "A Rapid Method Emplaylng Impregnated Charcoal and Atomic Absorption Spectrophotometry from the Determination of $\mathrm{Hg}$ in Atmospheric, Blological and Aquatic Samples," At. Abs. Newsletter 9, 113 (1970).

Perry, H., "Environmental Aspects of Coal Mining," in Power Generation and Environmental Change, D. A. Berkowitz and A. M. Squires, Eds. (The MIT Press, Canbridge, MA, 1971).

Putman, J. J., "QuicksIlver and Slow Death," Natlonal Geographic 124, (October 1972).

Stah1, Q. R., "Preliminary Air Pollution Survey of Mercury and Its Compounds," EPA Publication APTD 69-40 (USGPO, Washington, DC, 1969). 
Table 16. Major energy sources of trace metals in the air and their effects on human health.*

\begin{tabular}{|c|c|c|c|}
\hline Element & $\begin{array}{c}\text { Thireshold limits } \\
\left(\mathrm{mg} / \mathrm{m}^{3}\right)\end{array}$ & Sources & Effects on human health \\
\hline
\end{tabular}

\begin{tabular}{|c|c|c|c|}
\hline $\mathrm{Be}$ & 0.002 as $\mathrm{Be}$ & $\begin{array}{l}\text { Metallurgical Industry, } \\
\text { rocket fuels, and } \\
\text { coal }\end{array}$ & $\begin{array}{l}\text { Chemical pneumonitis, } \\
\text { berylliosis, chemical } \\
\text { ulcer, and carcino- } \\
\text { geresis }\end{array}$ \\
\hline$C d$ & $\begin{array}{l}0.2 \text { as } \mathrm{Cd} \text { and } \mathrm{Cd} \\
\text { in Its salts; } \\
0.1 \text { as CdO fume }\end{array}$ & $\begin{array}{l}\text { Electroplating, } \\
\text { alloys, solders, and } \\
\text { coal }\end{array}$ & $\begin{array}{l}\text { Pulmonary emphysema, } \\
\text { hypertenstan, kidney } \\
\text { damage, and carcino- } \\
\text { genesis }\end{array}$ \\
\hline $\mathrm{Hg}$ & $100 \mu g / \mathrm{m}^{3}$ & $\begin{array}{l}\text { Coal, geothermal } \\
\text { sources }\end{array}$ & $\begin{array}{l}\text { Hypertension, psychic } \\
\text { and emotional } \\
\text { disturbances }\end{array}$ \\
\hline $\mathrm{Ni}$ & $\begin{array}{l}1.0 \text { as } \mathrm{NI} \text { and } \mathrm{NI} \\
1_{11} \text { its salts; } \\
0.007 \text { as nickel } \\
\text { carbonyl }\end{array}$ & $\begin{array}{l}\text { Steel and nickel-alloy } \\
\text { Industry, nickel } \\
\text { plating, asbestos, } \\
\text { coal, fuel oil, and } \\
\text { incineration }\end{array}$ & $\begin{array}{l}\text { Dermatitis, respiratory } \\
\text { disorder, and } \\
\text { carcinogenesis }\end{array}$ \\
\hline $\mathrm{Pb}$ & 0.2 as $\mathrm{Pb}$ & $\begin{array}{l}\text { Automobile exhaust, } \\
\text { coal, incineration, } \\
\text { and pigments }\end{array}$ & Lead poisoning \\
\hline
\end{tabular}

*[Adapted from Hwang, J. Y., Ana1. Chem. 44, 21A (1972).] 


\section{Hydrogen Sulf 1 de}

Hydrogen sulfide gas is very toxic to humans and at concentrations over $1,000,000 \mu \mathrm{g} / \mathrm{m}^{3}$ quickly causes death by paralysis of the resplratory tract (Table 17). At lower concentrations it causes conjunctivit is with reddening and lachrymal secretion. respiratory tract irritation, pulmonary edema, damage to heart muscle, psychlc changes, disturbed equilibrium, nerve paralysis, spasms, unconsciousness, and circulatory collapse.

One outstanding eplsode In Mexico Involving accidental release of hydrogen sulfide killed 22 people and $50 \%$ of the exposed animals, and hospitalized 320 persons.

The gas has a very obnoxious odor at low concentrations ( 1 to $45 \mu \mathrm{g} / \mathrm{m}^{3}$ ).

\section{References - Hydrogen Sulfide}

White, D. E., W. P. Muffler, and A. H. Truesdell, "Vapor-Dominated Hydrothermal Systems Compared with Hot-Water Systems," Econ. Geol. 66, 75 (1971). 
Tavle 17. Effects of hydrogen sulfide on humans. *

\section{Coucentration $\left(\mu \mathrm{g} / \mathrm{m}^{3}\right)$}

Effects

$$
1-45
$$

10

150

500

15,000

30,000

$30,000-60,000$

150,000

$270,000-480,000$

$640,000-1,120,000$

900,000

$1,160,000-1,370,000$

$1,500,000+$
Odor threshold; no reported injury to health

Threshold of reflex effect on eye sensitivity to light

Smell slightly perceptible

Smell defintely perceptible

Minimum concentration causing eye irritation

Maximutn allowable occupational exposure for $8 \mathrm{~h}$ (ACGlH Tolerance Limit)

Strongly perceptible but not Intolerable smell; mintraum concentration causing lung irritation

01 factory fatigue in $2-15 \mathrm{~min}$; ti ritation of eyes and resplratory tract after $1 \mathrm{~h}$; death in 8 to $48 \mathrm{~h}$

No serious damage for $1 \mathrm{~h}$ but intense Lucal irritation; eye irritation in 6 to $8 \mathrm{~min}$

Dangerous concentration after $30 \mathrm{~min}$ or less

Fatal in 30 min

Rapid unconsciousness, respiration arrest, and death, possibly without odor sensation

Immedlate unconsciousness and rapid death

*[Miner, S., Air Pollution Survey of $H_{2}$, Department of Health, Education, and Welfare, National Air Pollution Control Assoctation, Raleigh, NC, Pub1. APTD 69-37 (1969).] 


\section{Water Vapor}

Plumes and fags from towers and ponds become an environmental hazard if they restrict visibility on streets, bighways or rallroads, or produce lcing (when the temperature is below freezing) on streets, sidewalks, or power lines. Vision-obstructing fogs may extend downwind 1500 ft or more fron wet cooling towers.

Problems of fog and icing have usually been associated with mechanicaldrait towers which release their effluent plumes relatively close to the ground (50 to $75 \mathrm{ft}$ ). The natural-draft, hyperbollc towers release their plumes so high, usually above $350 \mathrm{ft}$ that they seldom if ever sink to ground 1 evel.

The combination of vapor from cooling towers and stack gases from fossilfueled plants can cause special problems tnvolving the generation of actd droplets downwind from the plant.

Continual release of water vapor into the stratosphere might increase the prevalence of cirrus clouds, which could alter the earth's heat balance, however, the Iong-term climatic consequences of increases in the earth's high cloud cover are still unknown.

Stratosphertc water vapor will increase, on a global average, by 0.2 ppts by mass (from 3.0 to $3.2 \mathrm{Ppm}$ ). Since there will be more water vapor added to the north temperate latitudes, parts of this region may perhaps have a contribution to standing concentration as much as tenfold higher than the increase of the global average (that 1s, grow from 3 to 5 ppm water vapor).

Water vapor and molecular hydrogen released between $88 \mathrm{~km}$ and orbit insertion at $442 \mathrm{~km}$ by Skylab's rocket excavated a 1000-km hole in the tonosphere on May 14, 1973.

Water vapor in the stratosphere does not absorb much solar radiation. But it absorbs and entts strongly in the ir region of the spectrum and, by the "greenhouse effect," warms the lower atmosphere (Fig. 12). The present estimate of this warming effect is characterlzed by about the same uncertainty as the estimate of the cooling effect due to $\mathrm{sO}_{2}$. At this time, the water vapor effect is estimated to be about one-half the cooling effect due to the $\mathrm{SO}_{2}$ emitted using present-day fuels. Note that while the $\mathrm{SO}_{2}$ effect can be reduced directly by using fuel with a smaller sulfur content, there is no way to reduce water vapor emissions from an operating atreraft. 


\section{References - Water Vapor}

Huff, F. A., Effect of Cooling Tower Effluents on Atmospheric Conditions in Northeastern Illinois, Illinois State Water Survey, Circular 100, Urbana, IL (1971).

Stockman, J,, Cooling Tower Study, IITRI Report C6187-3 for the Environmental Prozection Agency (January 1971).

Veldhuizen, H., and J. Ledbetter, "Cooling Tower Fog: Control and Abatement," J. Air Poll. Cont. Assoc. 21, 21 (1971) .

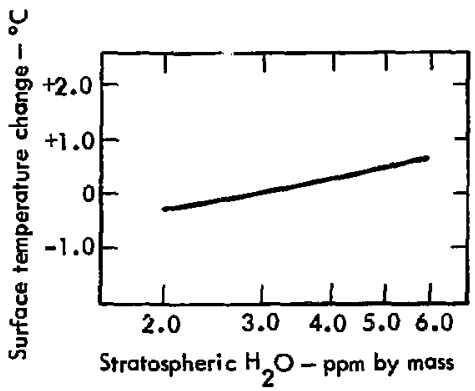

Fig. 12. Surface air temperature change due to increased stratospheric water vapor, with average cloudiness and corrective-radiative equilibrium. [Manabe, S., and R. T. Wecherald, "Thermal Equilibrium of the Atmosphere with a Given Distribution of Relative Humidity." J. Atm. Sc1. 24, 241 (1967).] 


\section{RADIOLOGICAL FACTORS}

\section{Gaseous Radioactivity}

The hazard of tritium is not from penetrating radialion, but from tritlum behaving chemically as hydrogen. There are tsotope effects on chemical kinetics and equilibria, but as far as we know, these are of minor importance in its radioroxicity. Tritium readily converts to tritfated water, HTO. In this chemical form it can quickly pass through the lungs and skin and become uniformly dispersed throughout the body fluids. For this reason, tritium that is chenically bound in water fs several hundred times more hazardous than tricium as an elemental diatomic gas. In addition, it can become Incorporated Into the cells of plants, both by Isotopic exchange and photosynthesis. The plants are eaten and tritium is Incorporated Into the molecular structures of our bodies.

Radioactive decay of tritium within the body has two damage mechanisms. one is the usual chemical changes induced by lonizing radiation. The 5.6-keV tritium beta particle can produce about 160 lonizations in tissue. In this respect, the hazard assoclated with tritium is substantially the same as other kinds of radiation, such as gama 1 ays when compared on a rad- $(100 \mathrm{erg} / \mathrm{g}$ tissue)-for-rad basis. The other mechanism results from a tritsum atom in a molecule (DNA, for example) which suddenly converts to a hellum atom. The molecule becomes a free radical and can react chemically in an abnormal biological fashion. Rats that have been glven doses of tritiated thymidine, a precursor of the DNA molecule, show a very high incidence of various cancers. In the studies done to date, however, this second mode of damage appears to be heavily overshadowed by the wore conventional damage mechanisms, and the effectiveness factor for tritium is only about 1.7 .

Since the noble gases are chemically inactive they remain in the atmosphere In elemental form until they decay and are not concentrated or retalned anywhere in the body. The health effects would be those due to gamma radiation. 


\section{References - Gaseous Radioactivity}

Baines, E. C., "Ionizing Radiation," In The Industrial Environment - Its Evaluation and Control (National institute for Occupational safety and Health, Washington, DC, 1973), Chap. 29.

Gofman, J. W., and A. R. Tamplin, IEEE Trans. Nucl. Science NS-17, 1 (1970).

Hlckman, R. G., Tritium In Nuclear Fusion Power, Lawrence Livermore Laboratory, Rept. UCRL-75546 (1974).

Report from the National Research Counctls Committee to Study the Long-Term Worldwide Effects of Multiple Nuclear Weapons Detonations, NAS-News Report (1975), p. 9 .

Schurr, S. H., Energy Research Needs, Resources for the Future, Inc., Washington, JC, Publ. PB-207516 (Octobe. 4971). 
The primary environmental effects arise when radioactive nuclear aerosols, resulting from atmosphere nuclear explosives, are precipitated or rained out on the earth's surface. Human exposure $\circ$ fallout is from three sources: ingestion, inhalation, and whole-bory cxternal exposure. Of the nuclides ingested, the main contributors to dase are ${ }^{90} \mathrm{Sr}$ and ${ }^{89} \mathrm{Sr}$ (to whole-body and to bone); ${ }^{131} \mathrm{I}$ (to thyroid); ${ }^{137} \mathrm{Cs}$ (to whole-body); and ${ }^{14} \mathrm{C}$ (to whole-body and to bone). Because strontium is deposited permanently in bone, the ${ }^{90} \mathrm{Sr}$ doses have been estimated based upon a 50-year (or lifetime) delivery of dose. The whole-body dose from inhalation is quite small; the 50-year respiratory 1 ymph-rode dose from plutonium is large indeed.

From a biological standpoint, the most important long-term fallout constituents are ${ }^{90} \mathrm{Sr}$ : and ${ }^{137} \mathrm{Cs}$, because of their long half-lives (28 and 30 years, respectively) and relatively high uptake and damage in biosystems. Strontium is chemically sirilar to calcium, winch is used for bone formation, while cesium simulates potassiun, crucial for the function of cells.

Estimates of ${ }^{90} \mathrm{Sr}$ intake via the total diet in New York City and San Francisco have been made since 1960 from quarter1y food samplings and average consumption statistics. The dietary incakes of ${ }^{90}$ sr have decreased from the maximum levels attained during 1963 and 1964 , but the declines have become more gradual in recent years due to the continuing small amounts of ${ }^{90} \mathrm{Sr}$ deposition and the 1fttle-changing cunulative deposit of ${ }^{90} \mathrm{Sr}$ in soil. The annual intake in 1973 averaged $9.7 \mathrm{pCi} /$ day in New York and $3.2 \mathrm{pCi} / \mathrm{day}$ In San Francisco, slight decreases from the previnus year. Further gradual reductions in ${ }^{90} \mathrm{Sr}$ intake are anticipated during 1974. 
Eberhardt, L. L., and R. O. Gilbert, Statistical Analysts of Soil Plutonium Studies, Nevada Test Site, Battelle Pacific Northwest LaLoratorles Rept. NBWL-B-217 (1972).

Gudiksen, P. H., C. L. Lindeken, C. Gatrovsis, and L. R. Auspaugh, Environmental Levels of Radioactivity in the Vicinity of Lawrence Livermore Laboratory, 1971, Lawrence Livermore Laboratory Rept. UCRL-51242 (1972).

Holladay, G., S. Bishop, P. Phelps, and L. R. Anspaugh, "A System for the Measurement of Deposition and Resuspension of Radioactive Particulate Released from Plowshare Cratering Events," IEEE Trans. Nuc1. Science NS-17, 151 (1970).

Krey, P. W., "Atmospheric Burnup of a Plutonfum-238 Generator," Science 158, 769 (1967).

Magno, P. J., P. E. Kauffman, and B. Shleien, "Plutonlum In Environmental and Biological Med1a," Health Physics 13, 1325 (1967).

Radionuclides in Surface Alr, USAEC Health and Safety Laboratory Rept. HASL-242 (1972), Append1x C.

Stern, A. C., Ed., Alr Pollution, Vol. 1: A1r Pollution and Its Effects, 2nd Edition (Academ1c Press, New York, 1968).

Volchok, H. L, , Fallout of Pu-238 from the SNAP-9A Burnup, USAEC Health and Safety Laboratory Rept. HASL-227 (1970).

Volchok, H. L., and R. H. Knuth, "The Respirable Fraction of Plutonium at Rocky Flets," Health Phys1cs 23, 395 (1972). 


\section{PHYSICAL FACTORS}

\section{Thermal Inputs}

The weather pattern of the classical air emissions eptsode is a prolonged pertod of near constant conditions: 1ittle or no wind or turbulence, temperature reduced both in mean level and diurnal range, high humidity accompanied by a several-hundred-foot layer of water-drop fog or low-level unbroken stratus clouds.

Practically all of the vital endocrinological and other physiological functions of the body are affected by sudden changes in environmental temperature modulated by humldity, wind or turbulence, and lighting. Sost adjustments are rapid and accommodate the diurnal cycle, sthers are of longer cerm and may be measurable over pertods of weeks.

Four aspects of the thermal regulating system are important to air emission episode problems:

- Morbidity Limit. The specific physiological condition of a healthy persin which leads to disease if this particular condtion is surpassed. This limit is temperature and humidity dependent, is different each day for different people, and differs in the same person from one day to the next.

- Age Dependence. The thermal regulatory mechanism is poor in both the very young and the aged. A correlation between heat waves, low cemperatures, and mortality of those under 1 and over 44 has already been noted, Alcoholism, arteriosclerosis, and metabolic diseases are cited as usual concomitant disturbances in the older heat-wave deaths.

- Psychosomatic Forces. The unconscious and conscious psychic life modulates the thermal regulating functions of the rhinencephalic hypothalmus-pituitary system.

- Prior Conditioning. The control and operational functions of the thermal regulating systens are highly dependent on seasonalization, thermal tempering, and previous physiological stimulus (W1lder's Law). 
Through the Interaction of the above factors something comparable to Wildt-'s Law of the Inftial value applies to populations as well as to an individual nervous system. Succeeding temperature extremes in the same season are less likely to cause mortality because of a longer period of seasonalization and through both thermal tempering and removal of susceptible Individuals by the preceding periods of stress. On the other hand, the lethality of a particular cold wave (heat wave) can be greatly enhanced by the survival, 1.e., accumulation in the population, of susceptible individuals through one or acre preceding mild winters (summers).

Statistics show that mortality spikes appear in association with early or preseasonal heat and cold waves even though the amblent temperature reached during such perfods may not be extreme. The fall and early winter rise in cardlovascular and respiratory mortality is, in general, steeper than the spring and early summer decline. These phenomena are particularly noticeable when the preceding contrasting season is extreme or prolonged and/or when the preceding like season is mild. Similarly, periods of extreme temperature occurring late in season or preceded by significant periods of similar temperature anomaly generally show only normal or even subnormal seasonal mortality. (Figures 13-15 and Table 18.)

\section{References - Thermal Inputs}

Andrews, C. H., "The Complex EpidemLology of Respiratory Virus Infections," Science 146, 1274 (1964).

Buckland, F. E., and D. A. J. Tyrrell, "Loss of Infectivity on Drying Various Viruses," Nature 195, 1063 (1962).

Chanock, R. M., "Control of Acute Mycoplosmal and Viral Resplratory Tract Disease," Science 169, 248 (1970).

Goldwasser, R., and T. J. Kligler, J. Prev. Med. 4, 361 (1936).

Huntington, E., "Weather and Health: A Study of Daily Mortality in New York City, Bulletin No. 75 (National Research Counc1l, National Acadeny of Sciences, Washington, 1930).

King don, K. H., "Relative Humidity and Airborne Infections," Am. Rev. Resplratory Disease:i 81, 504 (1960). 
Kutschenreuter, P. H., "A Study of the Effect of Weather on Mortaltty in New York City," Ph.D. thesis (Rutgers unfversity, New Brunswick, NJ, 1960).

Mudd, S., S. B. Grant, and A. Coldman, "The Etlology of Acute Inflammations of the Nose, Pharynx, and Tons1ls," Annals Otology, Rhinology and Laryngology 30, 1 (1921).

New York State Comnission on Ventilation, Ventilation (Dutton \& Co., New York, 1923).

North, C. E., Amer. J. Pub. Health 3, 222 (1913).

Proctor, D. F., "Physiology of the Upper A1rway," in Respiration, W. 0. Fenn and H. Rahn, Eds. (American Physiological Society, Washington, DC, 1964), Vo1. 1, pp. 309-345.

Scott, J. A., Report of the County Medical officer of Health and School Medical offlcer for the Year 1951 (London County Counc11, London, 1951).

Stuart Harris, C. H., "The Present Status of the Respiratory Viruses and Acute Respiratory Disease In Man," Israel 5, Med. Sc1. 2, 255 (1966).

Tromp, S. W., Medical Blometeorology (Elsevier Publishing Co., New York, 1963 ).

Winslow, C.-E. A., and L. P. Herrington, Temperature and Human Life

(Princeton University Press, Princeton, 1949). 
Table 18. Heat wave mortallty. *

\begin{tabular}{|c|c|c|c|c|}
\hline Year & Hot spel1 & $\begin{array}{c}\text { Average } \\
\text { daily deaths }\end{array}$ & $\begin{array}{l}\text { No. days } x \\
\text { daily excess }\end{array}$ & $\begin{array}{l}\text { Excess } \\
\text { deaths }\end{array}$ \\
\hline \multirow[t]{4}{*}{1949} & $20-27$ Jun & 234 & $8 \times 17$ & 136 \\
\hline & $19-22 \mathrm{Ju}$ & 223 & $4 \times 20$ & 80 \\
\hline & $27-30 \mathrm{Jul}$ & 241 & $4 \times 38$ & 152 \\
\hline & 7-12 Aug & 236 & $6 \times 45$ & 270 \\
\hline \multirow[t]{3}{*}{1952} & 15-18 Jun & 229 & $4 \times 12$ & 48 \\
\hline & 23-28 Jun & 320 & $6 \times 103$ & 618 \\
\hline & $13-24$ Ju1 & 239 & $12 \times 36$ & 432 \\
\hline \multirow[t]{5}{*}{1953} & 4-8 Jun & 251 & $5 \times 34$ & 170 \\
\hline & 19-23 Jun & 251 & $5 \times 34$ & 170 \\
\hline & 16-19 Jul & 233 & $4 \times 30$ & 120 \\
\hline & 24-31 Aug & 275 & $8 \times 84$ & \multirow{2}{*}{1002} \\
\hline & $1-5$ Sept & 269 & $5 \times 66$ & \\
\hline \multirow[t]{2}{*}{1955} & $1-9 \quad J u 1$ & 266 & $9 \times 63$ & 567 \\
\hline & $16-25 \mathrm{Ju} 1$ & 235 & $10 \times 32$ & 320 \\
\hline 1956 & 12-16 Jun & 288 & $5 \times 71$ & 355 \\
\hline \multirow[t]{2}{*}{1957} & 12-19 Jun & 276 & $8 \times 59$ & 472 \\
\hline & $20-24$ Jul & 262 & $5 \times 59$ & 245 \\
\hline
\end{tabular}

${ }^{*}$ [Kutschenseuter, P. H. Ph.D. thesis (Rutgers, New Brunswick, NJ, 1960).] 


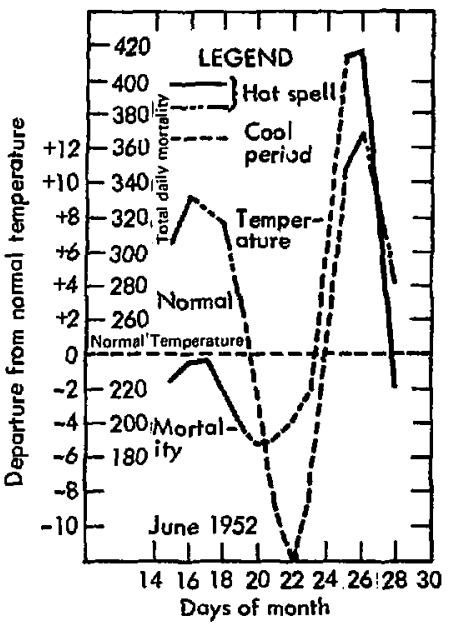

June

\begin{tabular}{|c|c|c|c|c|c|c|}
\hline Date & $T_{x}$ & $T_{n}$ & $T$ & $\Delta N T$ & & ind \\
\hline 15 & 93 & 68 & 81 & +10 & & \\
\hline 16 & 85 & 69 & 77 & +7 & SE & 6.0 \\
\hline 17 & 95 & 70 & 83 & +11 & SW & 8.4 \\
\hline 18 & 88 & 70 & 79 & +7 & $W$ & 10.2 \\
\hline 19 & 87 & 66 & 77 & +5 & $5 W$ & 10.7 \\
\hline 20 & 78 & 59 & 60 & -3 & NW & B.8 \\
\hline 21 & 64 & 59 & 62 & -11 & NE & 8.0 \\
\hline 22 & 62 & 58 & 60 & -13 & NE & 9.4 \\
\hline 23 & 64 & 58 & 61 & -12 & $E$ & 7.7 \\
\hline 24 & 85 & 62 & 74 & +1 & $S W$ & 7.0 \\
\hline 25 & 99 & 75 & 87 & +14 & S & 8.6 \\
\hline 26 & 100 & 81 & 91 & +17 & $w$ & 7.7 \\
\hline 27 & 90 & 72 & 81 & +7 & $N$ & 7.9 \\
\hline$x$ & $M$ & & $\mathrm{mo}$ & ature & & \\
\hline$n$ & $N$ & 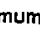 & 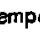 & Iture & & \\
\hline$T$ & $\mathrm{Me}$ & $t$ & & & & \\
\hline
\end{tabular}

$\triangle N T$ Departure from nomal temperature

Wind Prevailing direction and average hourly velocity
Fig. 13. Response of dafly mortality to temperature during heat waves that exceed minimum conditions. The conditions defining a heat wave which resulted in increased mortality (with a 1-day phase lag) were found to be: (a) three or more consecutive days with temperatures consistently above the seasonal normal and with 3-day mean departures from notmal $25^{\circ} \mathrm{F}$ and (b) maximum temperature on at least one day greater than $90^{\circ} \mathrm{F}$ and the departure from normal on at least one day $\geq 10^{\circ} \mathrm{F}$. [Kutschenreuter, P. H., Ph.D. thes1s, Rutgers Un1versity, New Brunswick, NJ (1960).] 

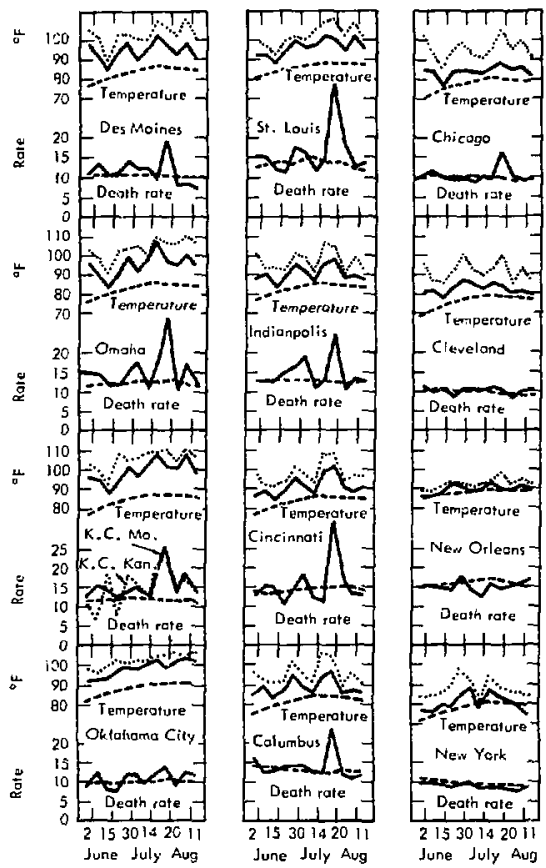

Temperature, of

- Weekly means of daily maximum temperatures in 1934 period.

- Norn.ol neekly mans of daily maximum temperature based on carres. ponding weeks of 40 ro 60 years.

- Actual weekly maximum temperature in 1936 period.

Death rate from atl causes per 1000 population (annual basis)

- Weekly rote in 1934 pariod.

- Average weekly rate for correponding weeks of 1930-1933.

Fig. 14. Week1y death rates from all causes and week1y temperature records in the summer of 1934 for nine cities in the drought area and three clites not affected by the heat wave. Simple averaging of the weekly death rates in the es.ght cities with definite mortality peaks (Ch1cago, Cincinnati, Columbus, Des Moines, Indianapolis, Kansas CIty, MO, Omaha, and St. Louis) gives a mean death rate of 25.5 per 1000 population (annuel basis) as compared with an expected normal rate for this season of the year of 12.4. This represents a weekly mortality excess of $106 \%$ due to hot weather for the combined populations of these eight citles. [Collins, S, D., Publif Health Reports 49, 1015 (1934).] 


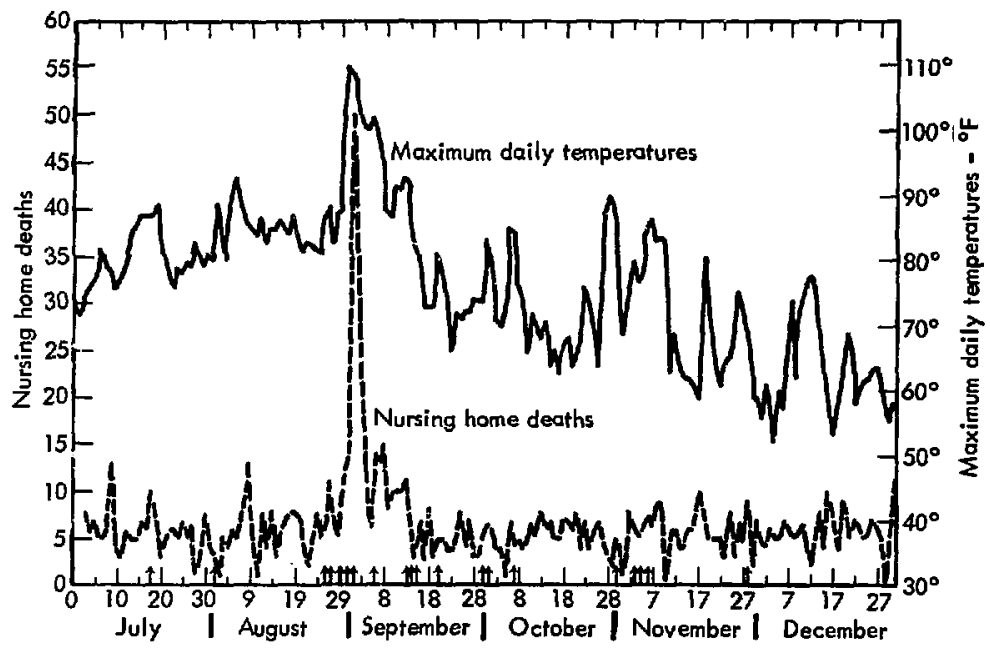

Fig. 15. Nursing home deaths, maximum daily temperatures, and smog alert days, Los Angeles County, California, July 1955 through December 1955. Unlike London's pollution, the worst Los Angeles smog attacks occur during hot weather. Some of the attacks occurred, for example, just prior to and following a heat wave in late August and early September in 1955. About 1000 excess deaths occurred during that period, but it is generally agreed that it was the heat (a week with maximal temperatures over $38^{\circ} \mathrm{C}$ ) that was the major contributor. $\uparrow$ indicates days of official alerts (ozone at $0.50 \mathrm{ppm}$ or higher). [State of California, Department of Pub1Ic Healch.] 


\section{Electromagnetic Emissions}

Radio- and Low-Frequency Radiation

An area of Investigation which is related to and often spoken of in the same context as electromagnetic waves and electromagnetic fields, 1s highfrequency electric currents. Their ability to heat tissue simulates the effect of electromagnetic waves. Consequently, high-frequency alternating currents have their medical application in diathermy, and have been erroneously given the name of "ultra-shortwave" (USW) therapy.

There is sufficlent evidence in the literature indicating that electromagnetic radiation in the radiofrequency and very-low-frequency range is capable of producing thermal effects which are, therefore, absorbed-dosage dependent as well as nonthermal effects which are fleld dependent and not necessarily related to heating. These effects are primarily concerned with central nervous system and pertpheral nervous systẹ.

A long-term study was made of these effects with systematic madical examinations of about 250 men working at 500 - and $750-\mathrm{kV}$ substations. These results were compared with medical examinations of men working at lower voltage substations. The studies showed that long-time work at 500-kV substations without protective measures resulted in changes of the dynamic state of the central nervous system, heart, and blood-vessel system, and in changing blood structure. The severity of these effects depended directly on the length of stay in the field.

Another study showed that some types of cardiac pacenakers are susceptible to disruption even from very small electromagnetic fields. These results have been confirmed by other experimencs. Currents as small as $50 \mu \mathrm{A}$ disrupt the operation of demand-type pacemakers, and currents of this size are running continuously in the bodies of people standing on the ground under the $765-\mathrm{kV}$ lines.

An experiment on nerve and brain tissue demonstrated that electric fields over a certain crftical intensity can affect the function of these very delfcate tissues. Brain organelles were found to be especfally sensitive to electrical effects. Nore research is needed to define the exact level at which these disruptive effects begin to occur. 
In another experiment, monkeys were exposed to electric fields in air of power-line frequency and there was a significant alteration of the interresponse time of the exposed animals. This change in time perception is belleved to be a very sensitive measure of conditions which, if long-term exposure occurred, would result in inportant behavioral and neurological changes.

\section{U1trasonic Waves}

It is suspected that the biological effects of ultrasonic waves are relaced to the local generation of heat. thether any cellular destruction is associated with cavity formation in tissues is not known.

Hearing loss and effects on the heart and blood vessels have been observed, such as vasoconstriction, that may affect the adrenal cortical function. Observations of the mother and fetus in the course of several thousand obstetric procedures did not indicate any hazard.

However, no lnng-term follow-up studies have been done on those receiving ultrasound therapy in medicine. Experimental blological studies at reasonably low exposuxe intensities (E.s opposed to cIInIcal studies) suggest strnngly that undetected biological damage is assoclated with diagnostic exposure.

Where ultrasound passes into the air, as in certain 1ndustrial applications, the probability of tissue damage is lower than in the case of direct contact, since the coupling obtaired between ultrasonic transducers and air is very poor.

The dangers of airborne hearing damage, systemic effects, and psychological effects necessitate caution even in the case of airborne ultrasound. Suvh alrborne ultrasound is sometimes emitted by certain machines, so that surveillance is necessary based on appropriate measurements.

\section{Microwave Radiation}

The biological effects generated by exposure to microwave radiation (F1g. 16) are usually designated as thermal or nonthermal in nature. Thermal effects are those Interactions which are caused by the heating of the blological specimen and can be duplicated using conventional heating techniques. 
Nonthermal effects are due to the direct interaction of the electromagnetic fleld of the incoming microwave radiation and the biological spectman and are more difficult to detect than the thermal effects.

The two organs of the body which are particularly sensitive to elevation In temperature are the testicles and the eyes (Table 19). Research on dogs, rabblts, and rats has shown that at $10 \mathrm{~mW} / \mathrm{cm}^{2}$ (power density in milliwatts per square centimeter), pathological damage to the testes Include a degeneration of the epttheliun lining of the seminiferous tubules, and a sharp reduction in the number of maturing spermatocytes. The reduction in testicular function due to the heating effect at $10 \mathrm{~mW} / \mathrm{cm}^{2}$ appears to be temporary and reversible.

Cataracts have been produced in the eyes of experimental animals. Several investigators have used the eyes of rabbits to establish threshold for cataract formation. For cw radiation, the threshold in rabbits for long-term exposire was measured to be approximately $100 \mathrm{~mW} / \mathrm{cm}^{2}$. Several cases of eye cataracts In man due to microwave exposure have been reported at power densities of the same order of magnitude."

Modeling studies Indicate that the human vestibular apparatus (the inner ear) may be responsive to low-level mlcrowave radiation via indirect, caloric stimulation.

The most of ten reported effects of low-level microwave radiation are neurological in nature (Table 20). Effects on animals include changes in EEG patterns, changes in the conditioned reflexes, alterations in sensitivity to light, sound, and olfactory stimulf, alterations in the blocurrents of the cerebral cortex, and changes in behavior. Many subjective symptoms in humans working around alcrowave equipment have been reported by USSR and Eastern European researchers.

Genetic effects have been reported by some Investigators. Exposure of chlck embryos to microwave radiation induced abnormal development, wille conventional heating to the same temperature did not cause abnormalities. In general, the abnormalities appeared to be caused by the inhibition of growth and cell differentiation. Abnormal development of the "mealworm" beetle pupae has also teen detected when exposed to microwave radiation. This study also indicates that the abnormalities were not due to thermal effects but due to the Inhibition of cell differentiatior caused by a direct Interaction with the electromagnet ic field. 
Theoretical calculations demonstrate that radiation levels from powerful VHF broadcasting stations could be high enough to cause concern over pacemaker Interference and electroexplostve devices under certain circumstances. Epigastric distress aw/or nausea may occastonally occur at as low as 5 to $10 \mathrm{~mW} / \mathrm{cm}^{2}$ and are most commonly assoclated withln the frequency range from $8 \times 10^{3}$ to $12 \times 10^{3} \mathrm{MHz}$.

As an added factor, the radiation may be up to 10,000 times higher than the levels which Soviet Bloc researchers belleve to have direct effects on the central nervous system and other biological functions.

\section{Laser Radiation}

The main biological effects of laser radiation are due to heating of the tissues and are essentially local. There may also be nonthermal effects, such as crythema of the skin due to photochemical reactions. No.uthermal effects occur mainly with high-power-density pulsed lasers. No genetic effects or late effects. such as skin cancer, have been reported.

The primary hazard from laser radiation is exposure of the eyt. Eye damage can range from mild retinal burns, wi' lictle or no loss of visual acuity, to severe lesions with loss of central vision, and ultimately to gross overexposure with total loss of the eye.

The other organ of concern, besides the eye, is the sikin. It is not as sensitive as the eye and, if damaged, is usua $11_{;}$more easily repaired. However, it may be als? subject to severe damage Irom las impact when energy densities approach several joules per square centimeter $\left(\mathrm{J} / \mathrm{cm}^{2}\right)$. Laser damage to the skin may range from a mild erythema to a surfase charring, and ultimately to a deep hole literally burned and blown into the skin.

Under certain circumstances, the organ of concern may also be the blood vessels or the underlying organs. Blood vessels may be easily occluded or cauterized by a laser bit.

\section{Ultraviolet Radiation}

The blological effects of uv radiation depend on the absorbed dose and on the wavelength and intensity of the radiation. They result mainly from 
the absorption of energy by the proteins and nucletc acids of the exposed organism. SkIn erythema and burn is produced by uv radiant energies, with wavelengths from about 100 to $300 \mathrm{~nm}$.

The extent of penetration by uv radiation is usually slight, except for such tissues as the lens and humors of the eye, so that the critical organs are usually the skin and eyes. Exposure of the surface layer of the skin to such radiation may result in erythema, skin cancer, rapid skin aging, and photosensitization.

In the case of the Eye, the radiation is absorbed primartly in the cornea and may produce lesions that could lead to blindness. Some portions of the uv sipectrun are mutagenic and may be lethal to cells:

- Keratitis in the eye (inflammation of the cornea) occurs predominantly at wavelengths below $330 \mathrm{~nm}$.

- Conjunctivitis in the eye. The action spectrum for this effect appears to be stallar to that for skin erythema.

- Lenticular fluorescence nccurs in the eye at wavelengths around $360 \mathrm{~nm}$ (black-11ght region). This is a fluorescence of the lens which occurs only during exposure and produces no adverse effects at exposure levels which are common today.

- Skin carclnogenesis can occur at extremely high exposure levels. Statistically, it is found most often in people exposed to the sun for large fractions of their Ilves.

Human sensitivity to sunlight is controlled by heredity. This is exemplifled by genetic deficiencles in melanin formation and thus the absence of tanning (as In Irish, Scottish, and Welsh peoples); deficiencies in cellular capacity to repair solar ridiation damage, as in the inherited disorder xeroderma pigmentosum; metabollc over-production of porphyrin (a natural photosensttizer); and altered tryptophal metabolism.

The wavelengths of sunlight below $320 \mathrm{~nm}$, those modulated by the presence of ozone in the stratosphere, are the most detrimental to biological systems (Fig. 17). Yet it is this same wavelength region of light that produces the essentlal vitamin in the skin of man - vitamin D. Thus, the situation is one of balance; sun11ght is necessary for life, yet in excess it is harmful. 
Currently three major enzymatic pathways are known for the repair of uv-induced damage to DNA:

- The damaged part of the deoxyribonuclefc actd molecule is restored to its functional state in place.

- The damaged sections of a DNA molecule are cut out and replaced with undamaged nucleotides to restore the normal function of the DNA.

- The damaged section of DNA is not directly repalred but is bypassed during replication.

While uv radiation produces very few breaks dfrectly in DNA molecules, In the process of repatring uv-radiation-iuduced damage to DNA, enzymeInduced breaks are produced in the DNA. These breaks are then subsequently repaired.

The therapeutic value of uv radjation has been recognized for a long time. "Rest and sunshine" has been prescribed for ills since prehistory. Classic examples of the medical use of uv radiation are the treatment of tuberculosis and rickets.

Ultraviolet light's germicldal properties are employed in hospitals and operating rooms and In restrooms. Ultraviolet radiation can kill or inactivate viral strains which are responsible for influenad and other upper respiratory infections in humors.

The assessment of the changes caused by impact of increased solar uv on humans is complicated by the nature of the organisms, the water body under tudy, and the quality of water which is considered tolerable.

There can be no reasonable doubt, on the basts of animal experiments and human epidemiologic data, that frequent and prolonged exposure to sunlight is the major cause of basal-cell and squamous-cell carcinoma in man, and that the shortest uv wavelengths penetrating the atmosphere are matnly Iesponsible.

The most conservat Ive estimates suggest that a $5 \%$ decrease in ozone would result in an eventual increase of from 30,600 to 44,000 additiond cases of skin cancer per year (approxtmately 10 to $15 \%$ of the present inc"dence rate). 
Under these fleld conditions more subtle yet Important physlologlcal processes were altered. Mutation rates of stamen halrs on a special test Ine of Tradescantla were signiflcantly enhanced by the higher-uv-radiation regime. Also, ultrastructural modification of chlcroplast membranes (as shown by electron microscopy) was also apparent in soybean leaves exposed to the enhanced uv radiation in the fleld.

A 400-W high-intensity mercury vapor discharge lamp, whose outer uv absorbing envelope is broken, emits sufflcient radiation to cause severe photokeratitis and facial erythema in humans at distances of more than $20 \mathrm{ft}$.

Ultraviolet radiation from the sun is usually of no 1mportance, since the natural ozone layer in the stratosphere absorbs the most damaging wavelengths. However, it has been estimated that if the ozone layer were depleted $0.5 \%$ by energy-related chenical emisstons, skin cancer occurrence anong whites would increase $1 \%$.

\section{Vistble Light}

Because of tis narrow depth of penetration, vistble light in general does not manifest 1 tself as a potential hazard. However, there are situations in which it can become hazardous. Probably the one of greatest danger is that of high-intensity light which may cause (1) transient loss of visual function (flash blindness) or (2) irreversible thermal injury of the retina,

Flash blind hess is a relatively new problem to man accurring only since man has developed sources of light brighter than the sun. It results from the bleaching of the visual pigments. Flash blinding can be prevented with proper filtering of light when the occurrence is expected. The second of these effects on the eye, Irreversible thermal infury, has been known for years. For example, lookouts for afrplanes have on occasion suffered retinal lestons which appear to be caused by heat generated at the sun's Inage on the retina.

W1th regard to the eye there appear to be three predominant factors controlling the potential of the hazard: (1) Intensity, (2) pupil dilation (1.e., area of exposure), and (3) length of exposure. It these factors can be controlled to keep the absorbed energy below the threshold of thermal burning, reported to be between 40 and $50 \mathrm{sal} / \mathrm{cm}^{2} \cdot \mathrm{min}^{-1}$, no eye injury would be expected. 
Directly beneftcial effects of light produced by artifictal sources Include the photorepair of uv damage, treatment of jaundice of the newborn Infant with blue light, destruction of the virus of herpes stmplex when stained by certain photosensitizing dyes, diagnosis of some hereditary diseases in utero by activation of fluorescent dyes in fetal cells, and, recently, the destruction of certaill cancer cells by visible light Irradiation after thelr Incorporation of photosensitizing agents.

The psychological effects of light, particularly of colored $1 \mathrm{fght}$, are well known but not well understood. These effects may bear a causal relationship to purely btological processes in the brain induced by light, which in turn will affect psychic behavior. Light intensity as well as wavelength speciflcity may alter productivity and mood. In the Infant, sensory overload by prolonged exposure to highly intense illumfnation may produce undesirable effects on development. Indeed, the manipular Iun of the lighting environment of adults as well as of Infants can have conseyuences of which we may be quite unaware.

The penetration of visible light in tissues deeper than the skin has not been adequately measured. The penetration of uv radiation has been measured with some success, so that we do know much abcut the photochenical actions within these superficial cells, but the photochenical action of vistble light upon deep tissues is an area of scudy that neods much further work.

\section{Ef fects of Near Ultraviolet Light (320 to $400 \mathrm{~nm})$.}

The wavelengths of sunlight which are modulated by the ozone in the stratosphere, 1.e., 280 to $320 \mathrm{~nm}$, are the wavelengths of light that produce sunburn and skin cancer. Wavelengths longer than $320 \mathrm{~nm}$ are very ineffective in this regard. However, if both types of 1 ight are used together they produce a greatly enhanced sunburn reaction in human skin.

\section{Electromagnet1c Fleld Radiation}

In one study, 10 linemen who did repair work on 345-kV 1 lnes were checked throughout a 9 -year perlod with 7 medical examinations. At the end of that time 3 of the 10 linemen had a reduced sperm count; however, it would be difficult to draw any conclusion on this effect from such a small sample. 
In 1962, after the first 500-ky lines had been operating in the Soviet Unton for several months, men working at the substations began to complain of headaches and a general feeling of malajge. Abnormal fatigue and sleeplness mentioned by a majority of the workers were associated with exposure to the electric fields.

\section{References - Electronagnetic Enissions}

Coldwell, M. M., et al. Third Conference on CIAP, Department of Transportation (February 1974).

Calkins, John, Third Conference on CIAP, Department of Transportation (February 1974).

Cline, M. G., and R. B. Salisbury, Nature 211, 484 (1966).

Coate, W. B., et al., Project Sanguine: Blological Effects Test Progran, Final Report, report prepared for U. 5. Department of the Navy, Naval Electronics Systems Command Headquarters, Fall Church, VA, Hazelton Laboratories (November 1970).

Cogan, D. G., and V. E. KInsey, "Action Spectrum of Keratitis Produced by Uleraviolet Radiation," Arch. Ophthalmo 1. 36, 670 (1946).

Department of Navy, Electronic Systems Command, SanguIne Syscem Blological/ Ecological Research Program: Sumnary Stacus Report, Department of Navy, Waskington, DC (Apr11 1973), p. 3 .

Energy Research Reports (March 1976), p. 5.

Environment Report (October 11, 1974), P. B6.

Furman, Seymour, et al., "The Influence of Electromagnetic Environment on the Performance of Artificial Cardiac Pacemakers," Annals of Thoractc Surgery 6, 90 (July 1968).

IEEF Working Group on Electrostatic Effects uf Transmission Zines, "Electrostatic Effects of Overhead Transmission Lines," IEEE Trans. PAS-91, 437 (March-Apri1 1972).

Jackson, S. J., Effects of Microwave Radiation, National Library of Medicine, Department of Health, Education, and Welfare, Washington, DC, Literature Search No. 75-19.

Kouwenhoven, W. B, , O. R. Langworthy, and M, L. Singewald, "Medical Evaluation of Man Working in AC Electric Flelds," IEEE Trans. PAC-86, 510 (Apri1 1967).

Lebovitz, R. M. The Sensitivity of Portlons of the Human Central Nervous System to "Safe" Levels of Microwave Radiation, Rand Corporation, Santa Monica, CA, Publ. AD-756608 (March 1972). 
Marino, A. A., et al., "Electric Field Effects In Selected Biological Systems," Transactions of the New York Academy of Sciences (forthcoming).

Meese, W. J., Chairman, ANSI Standards Committee on Electric Safety Code, Nationa1 Bureau of Standards, to Louise B. Young, May 5, 1970.

Nature 241, 291 (1973).

Pierluissi, J. H., Effects of Electromagnetic Fields Below $30 \mathrm{MHz}$ On Animal Blology, Lawrence Livermore Laboratory, Rept. UCRL-51880 (1975).

Pitts, D. G., et al." The Effect of Ultraviolet Radiation on the Eye, USAF School of Aerospace Medicine, Brooks AFB, Publ. SAM-TR-69-10 (1969).

Riesen, W. H., et al., "A Pilot Study of the Interaction of Extremely Low Frequency Electromagnetic Elelds with Brain Organelles," IIT Research Institute Project E6185, technical memo No. 3, Naval Electronfcs Systems Command, Washington, DC (1974).

Roth, E. M., Ed., Compendium for Development of Human standards in Space System Design, Lovelace Foundation, Albuquerque, NM (September 1967).

Schwon, H. P., IEEE Trans. Blomedical Eng. BME-19, 304 (1972).

Science Trends 33 (March 3, 1975).

Smith, K. C., BloScience 24, 45 (1974).

Stone, G., Letters to the Editor, Aviation Week \& Space Technology 100, 58 (July 1, 1974).

TB MED 270/AFM 161-7, Department of the Army and Air Force (December 1965), p. 4.

Urbach, Frederick, et al., Third Conference on CIAP, Department of Transportation (February 1974).

Valentino, A. R., D. A. Miller, and J. E. Bridges, Susceptibility of Cardiac Pacemakers to 60 Hertz Magnetic Fields, IIT Research Institute jeport No. 737237, Department of Commerce, National Technical Information Service, Washington, DC (1972).

Wilkening, G. M., "Non-Ionizing Radiation," In The Industrial Environment Its Evaluation and Cont:ol (National Institute of occupational Safety and Health, Washington, DC, 1973), Chap. 28. 
Table 19. Thermal-blological effects of microwaves. *

\begin{tabular}{|c|c|c|c|}
\hline $\begin{array}{l}\text { Frequency } \\
(\mathrm{MHz})\end{array}$ & $\begin{array}{l}\text { Wavelength } \\
\text { (cm) }\end{array}$ & $\begin{array}{l}\text { Site of major } \\
\text { tissue effects }\end{array}$ & Major blological effects \\
\hline$>10,000$ & $<3$ & Sk1n & $\begin{array}{l}\text { SkIn surface acts as } \\
\text { reflector or absorber } \\
\text { with heating effects }\end{array}$ \\
\hline 10,000 & 3 & Skin & $\begin{array}{l}\text { Skin heating with sensation } \\
\text { of warmth }\end{array}$ \\
\hline 10,000 to 3,300 & 3 to 10 & $\begin{array}{l}\text { Top layers of } \\
\text { skin, lens } \\
\text { of eye }\end{array}$ & $\begin{array}{l}\text { Lens of eye and testicles } \\
\text { particularly } \\
\text { susceptible }\end{array}$ \\
\hline 10,000 to 1,000 & 3 to 30 & Lens of eye & $\begin{array}{l}\text { Critical wavelength band } \\
\text { for eye cataracts and } \\
\text { testicular damage }\end{array}$ \\
\hline 1,200 to 150 & 25 to 200 & $\begin{array}{l}\text { Internal body } \\
\text { organs }\end{array}$ & $\begin{array}{l}\text { Damage to internal organs } \\
\text { from overheating }\end{array}$ \\
\hline$<150$ & Above 200 & & $\begin{array}{l}\text { Body is transparent to } \\
\text { waves above } 200 \mathrm{~cm}\end{array}$ \\
\hline
\end{tabular}


Table 20. Clinical manifestations of chronic occupational exposure of humans to microwave radiation.*

\section{Neurological effects}

1. Bradycardia

2. Disruption of the endocrine-humoral process

3. Hypotension

4. Intensffication of the activfty of thyrofd gland

5. Exhausting Influences on the central nervous system

6. Decrease in sensitfvity to smell

7. Increase fn histamfne content of the blood

\section{Subjective complaints}

1. Increased fatigab1lity

2. Periodic or constant headaches

3. Extreme 1rritability

4. Sleepfness during work

\section{Central nervous system of antmals}

1. Changes in the conditioned reflexes

2. Alterations in sensitivity to 11 ght, sound, and olfactory st1mu11

3. Changes in structure of skin receptors of the digestive and blood carrying system

4. Alteration in the biocurrents of the cerebral cortex

5. Reversible, struccural changes in the cerebral cortex and diencephalon

6. Appearance of varlous vegetative reactions

\footnotetext{
${ }^{\star}$ [McRee, D. I., 3. A1r Poll. Cont. Assoc. 24, 122 (1974).]
} 

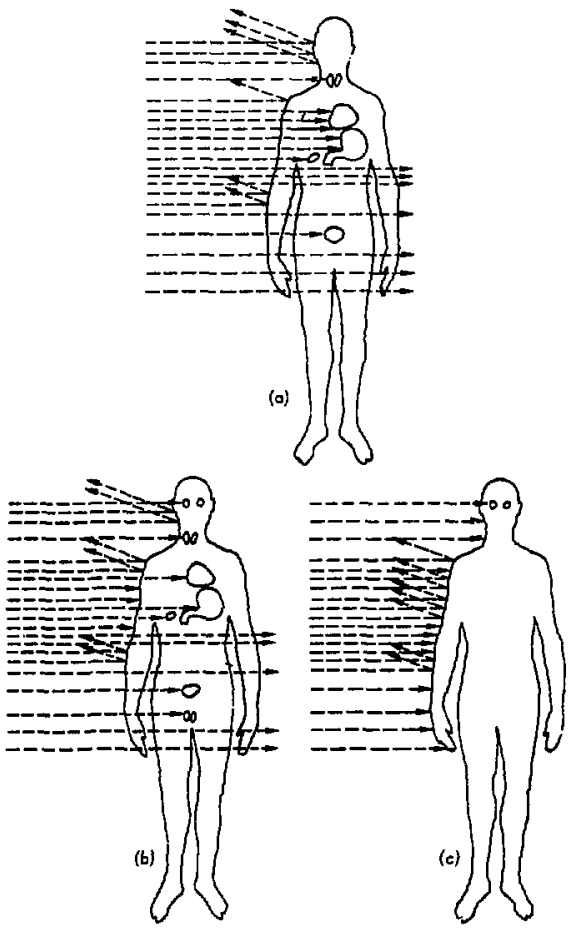

PIg. 16. Parts of the body affected by microwave radiation. (a) Radiation at frequencies of 150-1000 $\mathrm{MHz}$ is absorbed by Internal body argans shown. Approximately $40 \%$ of the energy is absorbed by the deep organs; the rest is either reflected or passes harmlessly through the body. Overheating and danage to organs with few blood vessels, such as the gall bladder, urinary bladder, and portions of the gastrointestinal tract, can result frum microwave exposure in this range. (b) Radiation at frequencies of 1000-3000 $\mathrm{MHz}$ is absorbed by both the skin and deep organs. The lens of the eye is particular 1 sensitive to $3000-\mathrm{MHz}$ energy. From 20-100\% of the microwave energy absorbed, depending on the fat and water content of the tissues hit by radiation. (Fat is a poor absorber of microwave radiation,) The remaining radiation is either reflected or transmitted.

(c) Rt lation at frequencles of $3,000-10,000 \mathrm{MHz}$ is absorbed only by the top layers of skin and the lens of the eye. Approximately $40 \%$ of the microwave energy is absorbed; the rest is reflected. [Aaransen, T., Environment 12, 2 (1970).] 

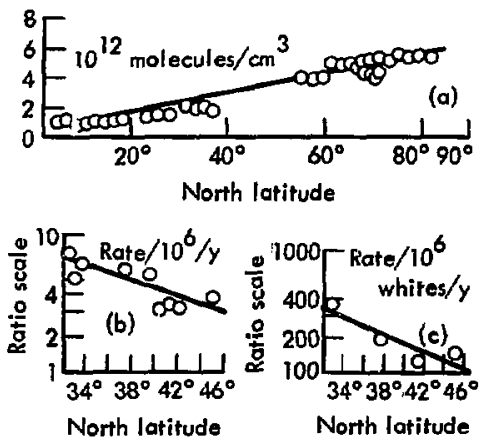

Fig. 17. Comparison of atmospheric ozone content with incldence of skin cancer and melanoma. Skin cancer and melanoma among whites decreases in the higher latitudes as the atmosphere ozone content Increases. (a) Variation of ozone with latitude at height of $11.4 \mathrm{mf}$. (b) Varlation of melanomi with latitude. (c) Variation of skin cancer (apart from melanoma) with latitude. The role of atmospheric ozone in protecting susceptible whites from uv radiation and skin cancer is believed tliustrated by the combination of a decrease in such cancer at higher latitudes and a rise In ozone quantity. The ozone measurements were made last year by $\mathrm{v}-2$ plane. Cancer Incidence is for 1970 In the U.S, and Is age adjueted. [The New York Times, February 7, 1975, P. 8. Copyright 1975 by the New York Times Company. Reprinted by permission.] 


\section{Notse}

General Health/Soclal Impact

Noise-induced hearing loss is the most significant physical health problem from exposure to excessive notse. It is eatimated that in 1975 7 million industricl workers in the U.S. were exposed to occupational notse levels that could damage their hearing. Lower levels of nolse encountered In the everyday environment of the 130 million residents of metropolitan areas may also cause some degree of hearing loss (FIg. 18).

Noise produces physical and mental stress, at least in some people; more than half the people responding to aurveys in major U.S. metropolitan areas admit to being annoyed by the levels of notse to whtch they are exposed. The complexity of the problem of annoyance indicates that progress will continue to be slow and that no easy or Immediate solution is probable.

\section{Pathological Effects of Nolse}

Although there is no doubt that noise causes annoyance and may adversely affect well-being, there is as yet no clear eptdemfological proof that the various physiological and psychophyslological responses to nolse will, in the long term, lead to pathologlcal changes. Long-term comparative studies are needed in order to gain a fuller understanding of this problem. Deafness is, of course, the major hazard Involved in exposure to noise that is too intense or too prolonged.

Deafness may occur suddenly due to acoustic trauma following exposure to very-hdgh-intensity nolse, but more usually appears gradually as a result of repeated exposure to nolse. The most typical case is that of occupational deafness, in the early stages of which hearing tmpatrment, as determined by audiometric methods, occurs ta the 3000- to 6000-Hz octave bands. The individual concerned is in most cases unaware of this impairment. Damage then becomes more serlous and 1 ts extent tnireases; it becomes particularly troublesome wher the voice frequencles are affectea. Impairment progresses in an Irregular manner toward severe deafness, assoctated with very high levels of mean hearing loss. Although there are some simflarities between effects, such as temporary threshold shift, auditory fatigue, and acoustic trauma, 
no conclusive evidence exists of any definite relationship between them.

Although the immediate effects of notse exposure on some body functions, such as pupillary dilation, pertpheral circulation, and respiratory changes, are reasonably well understood, the practical implications on the general heaith of the average individual have not been determined. Generally, humans adapt very well to stimul1 such as nolse, and most extra-auditory response changes measured appear to be transitory or short-term alterations.

Perhaps of greater importance is the question concerning possible adverse effects due to regular long-term exposure to nolse over many years. With the exception of hearing, evidence is not sufficient to permit us to determine if long-term exposure on a regular basis is either harmless or potentlally harnful to healih. Consequently, it may be wise to proceed on the assumption that long-term exposure might bly be harmful to health and well-being and to continue under this assumbion untij It can be proven ot rise.

Psychological and Phystological Effects

It has not been demonstrated that people are having their 1 ives shortened by exposure to audible noise. Perhaps the stress of crntinued exposure to high levels of nolse can produce disease or make us i. ce susceptible to disease, but, overall, the evidence is not convincing.

The elfects of nolse on people have not been successfully measured in terus of excess deaths. shorteued I1fespan, or days of Incapactlating IlIness. There are only hints that such effects might exist. Of course, theri lay be accidental deaths or injurles because varning signals were not heard or were misunderstood because of nolse.

There is clear evidence that exposure to nolse of sufficient intensity and duration can:

Permanently damage the Inner ear with resulting permanent hearing losaca that can range from slight impairment to nearly total deafness.

- Rebult in temporary hearing losses; repeated expoaures to nolse can result in chronic hearing losses. 
It is also apparent that notse can (FIgs. 19-22 and Table 21):

- Interfere with speech communication and the perception of orher auciltory signals.

- Disturb sleep.

- Be a source of annoyance.

- Interfere with the ability to perform complicated tasks and, of course, can especially disturb those tasks that demand speech communtcation or response to auditory signala.

These latter effects are difficult to quantify, alnce they affect the essential nature of human life - its quality. But alone they are sufficient to require more efforts toward controlling the problem.

\section{References - Noise}

Burrows, A. A., and D. M. Zamarin, "Aircraft Noise and the Communty: Some Recent Survey Findings," Aerospace Medicine (January 1972).

Envirunmenta1 Health Problems, Department of Health, Educarion and Welfare, Wasington, DC (1970).

Fif th Annual CEQ Report (USGPO, Hashington, DC, December. 1974).

Nixon, C. W., Some Effects of Noise in Man, Aerospace Medical Research Laboratory, Wright-Patterson AFB, OH, Publ. AMRL-TR-71-53 (1971). 
Table 21. Subjective responses to varfous afrbone ultrasonic exposures generated by ultrasonic instrumentation **

\begin{tabular}{|c|c|c|c|c|c|c|c|c|}
\hline \multirow{2}{*}{$\begin{array}{l}\text { Ultrasonic } \\
\text { Instrument } \\
\text { type }\end{array}$} & \multirow{2}{*}{$\begin{array}{c}\text { Operating } \\
\text { characteristic', }\end{array}$} & \multirow[b]{2}{*}{ Subjective response } & \multicolumn{6}{|c|}{$\begin{array}{c}\text { Sound pressure levels (dB) at midpoint } \\
\text { frequenctes (third octave bands) } \\
(\mathrm{kHz})\end{array}$} \\
\hline & & & 10 & 12.5 & 16 & 20 & 25 & 31.5 \\
\hline Drild & $\begin{array}{l}\text { Input freq. }-23.1 \\
-26.1 \mathrm{kHz} 400 \mathrm{~mA}\end{array}$ & $\begin{array}{l}\text { Pain in ears after few } \\
\text { minutes. Headache after } \\
1 \mathrm{~h} \text {. Nausea after } 2 \mathrm{~h} .\end{array}$ & 91 & 85 & 90 & 81 & 107.5 & 84 \\
\hline Welder & $\begin{array}{l}\text { Input freq, } \\
-15,750 \mathrm{~Hz}, \\
\text { intermittent } \\
\text { exposure, on } 1.5 \\
8 \text {, off } 5 \mathrm{~s} \\
1 \text { transducer }\end{array}$ & $\begin{array}{l}\text { Malaise, backache, } \\
\text { individual wore earplugs } \\
\text { and symptoms disappeared, } \\
\text { operator had a cold when } \\
\text { symptoms were experienced. }\end{array}$ & 76 & 95 & 115 & 93 & 73 & 69 \\
\hline Cleaner & $\begin{array}{l}\text { Input freq. } \\
-20 \mathrm{kHz}\end{array}$ & $\begin{array}{l}\text { Operator didn't use the } \\
\text { machine, the machine } \\
\text { nolse was so intense. }\end{array}$ & 125 & 123.5 & 119.5 & 113 & 133.5 & 110 \\
\hline Cleaner & $\begin{array}{l}\text { Input freq. } \\
-40 \mathrm{kHz}\end{array}$ & $\begin{array}{l}\text { Headache, malaise, } \\
\text { fatigue, when necessary } \\
\text { to use, the operator } \\
\text { leaves the room. }\end{array}$ & 77 & 78 & 79 & 96 & 77 & 77 \\
\hline Cleaner & $\begin{array}{l}\text { Input freq. } \\
-20 \mathrm{kHz} \\
1 \text { transducer, } \\
\text { water circulating } \\
\text { at } 20^{\circ} \mathrm{C}\end{array}$ & $\begin{array}{l}\text { Fatigue and malaise. } \\
\text { Shrill sound of } 94 \mathrm{~dB} \\
\text { at } 4 \mathrm{kHz} \text { midpoint } \\
\text { frequency. }\end{array}$ & 81 & 78 & 84 & 93 & 76 & 76 \\
\hline
\end{tabular}

*[Johnson, Lavern, "Auditory Response Threshoids and Habituation During sleep," paper presented at the 1971 annual Meeting of the Committee on Hearing, Bloacoustics, and Biomecharics, Cocoa Beach, Florida, March 31, 1971.] 
Highway vehicles (typical number of hours use per day on doy of use)

Motacyseles (I)

Medium and heavy trucks (4)

Highway buses (4)

Utility trucks (1)

Light trucks (1.5)

School and city buses (2)

Passenger cars, all types (1)

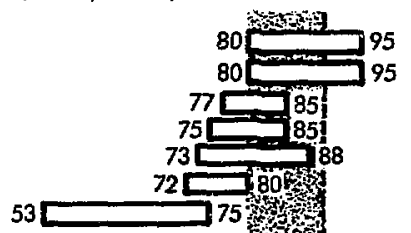

Aircraft

Light utility helicopters (2)

Commercial, propeller (1.4)

General aviation, propeller (1)

Commercial, 2- and 3-engine turbofan (1.4)

Heavy tronsport helicopter $(0.5)$

Medium weight helicopter $(0.5)$

Commercial, 4-engine turbofan

Commercial, widebody (1.4)

General aviation, execufive jet $(0.5)$

Rail vehicles

Rapid transit (1.5)

Trolleys (1.5)

Passenger trains (6)

High-speed interurban (2)

Recreational vehicles (typical)

Snowmobiles (2)

Minicycles and off-road motorcycles (2)

Inboard and outboard boats (2) 


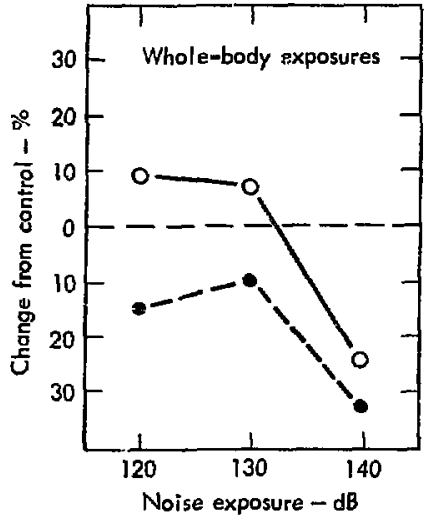

Fig. 19. Performance on a postural equilibrium task during whole-body (chamber) and auditory (earphone) exposures to broadband notse. Asymmetrical exposures used two hearing protectors (earpivg and earmuff) at one ear to provide about 20 dB less nolse and only one hearing protector (earplug) in the other ear. Symetrical exposures employed only earplugs in both ears. [Nixon, C. W., C. S. Harris,

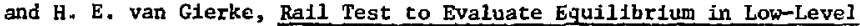
Wide Band Notse, U.S. Alr Force, Aerospace Medical Research Laboratiory, Wright-Patterson Afr Force Base, Oh1o, Rept. No. AMRL,Th-66-85 (1966).] 
Fig. 20, Representative low-frequency and Infrasonic noise environs and buman subjective responses to the exposures. [Mohr, G. C., J. N. Cole, E. Guild, and H. E. von Gierke, "Effecis of Low Frequeacy and Infrasonic Notse on Man," Aerospace Medicine 36, 9 (1965).]

\begin{tabular}{|c|c|}
\hline \multicolumn{2}{|c|}{ Toleran ce dato } \\
\hline Exiposure & Ocserved behovior \\
\hline $\begin{array}{l}0-50 \mathrm{~Hz} \\
\text { Up to } 145 \mathrm{~dB}\end{array}$ & $\begin{array}{l}\text { Chest wall vitmation, } \\
\text { gag sensations, res- } \\
\text { piratory rhythm } \\
\text { changes, postexposure } \\
\text { fatigue; voluntary } \\
\text { tolerance not } \\
\text { exceeded }\end{array}$ \\
\hline $\begin{array}{l}50-100 \mathrm{~Hz} \\
\text { Up to } 154 \mathrm{~dB}\end{array}$ & $\begin{array}{l}\text { Headoche, choking, } \\
\text { coughing, visual } \\
\text { blurring, fatigue; } \\
\text { voluntary tolerance } \\
\text { limit reached }\end{array}$ \\
\hline $\begin{array}{l}\text { Discrete } \\
\text { frequencies }\end{array}$ & $\begin{array}{l}\text { Tolerance limir } \\
\text { symptoms }\end{array}$ \\
\hline $\begin{array}{l}100 \mathrm{~Hz} \\
\text { af } 153 \mathrm{~dB}\end{array}$ & $\begin{array}{l}\text { Mild nousea, giddiness, } \\
\text { subcostal discomfort, } \\
\text { cutoneous flushing }\end{array}$ \\
\hline $\begin{array}{l}60 \mathrm{~Hz} \text { at } 154 \mathrm{~dB} \\
73 \mathrm{~Hz} \text { at } 150 \mathrm{~dB}\end{array}$ & $\begin{array}{l}\text { Coughing, severe sub- } \\
\text { sternal pressure, } \\
\text { choking respiration, } \\
\text { salivation, pain on } \\
\text { swollowing, giddiness }\end{array}$ \\
\hline
\end{tabular}



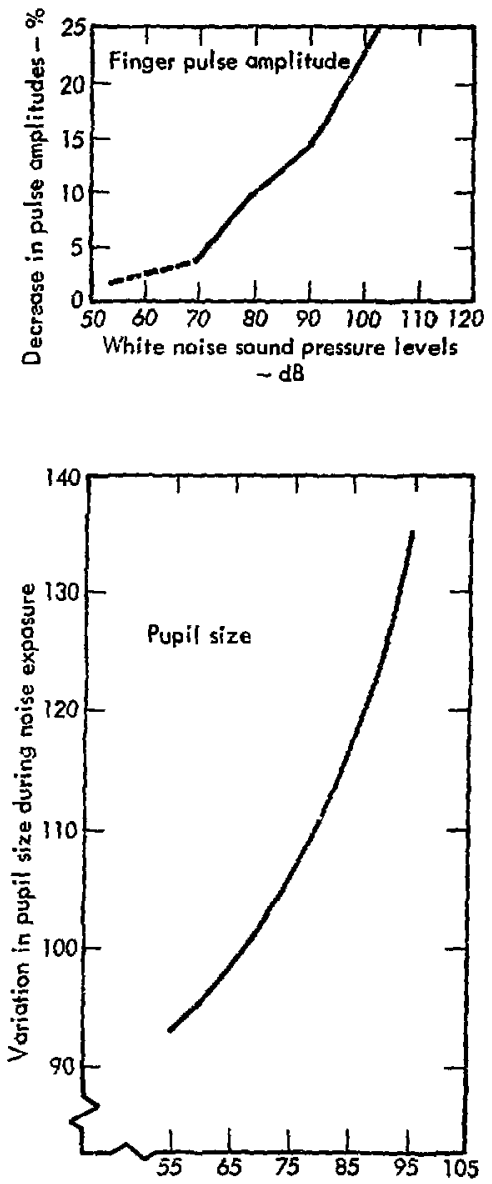

White noise sound pressure levels $-d B$
Fig. 21. Varlations tn amplitude of the finger pulse and in pupil size of subjects exposed to white noise at several different soundpressare levels. [Jansen, G. Noise as a Public Health Hazard, OSHA Publication No. 4 (USGPO, Washington, bi, 1969).] 


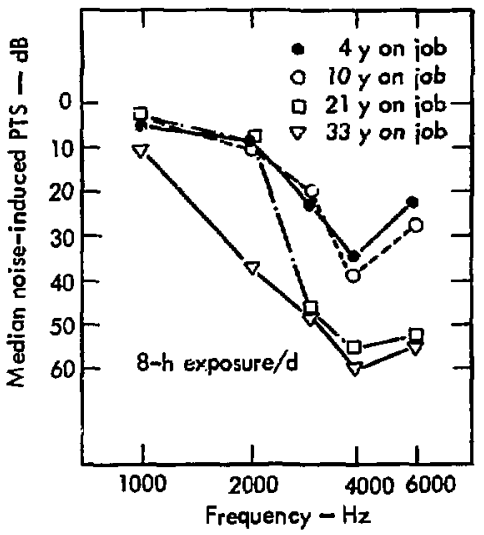

Fig. 22. Amount of noise-induced permanent threshold shift (PTS) experienced in groups of men who worked from 4 to 33 years in noise environments. [Glorig, Aram, W. Dixon Ward, and James N1xolt, "Damage Risk Criteria and Nofse Induced Hearing Loss," Arch. Otolaryngology 74, 413 (1961).] 


\section{Magnet fe pigld inlssions}

The human comaunity has been living with 60-Hz magnetic fields of moderate, but ever increasing, intensity for over 50 years without any demonstrated cases of infury, disabilfty, or even noticeable discomfort. Therefore, it is very unlikely that weak magnatic fields are ding anything very injurious to us individually or as a populetson. Studies in Eurupe. Asta and histralia wth 50-Hz magnet ic flelds and sevrral other frequencs-y show a simflar lack of noticeable ef fect.

However, human subjects can, under some conditlons, sense the presence of a 60-Hz magnetic field. Researchers have reported a wide "arlecy of such effects, ranging from interference with norwal embryonic development of frogs, to a statistical relationship between psychlacric disturbances and natural magnetic [ield intensity. Major blologica\} effects of a basfe nature have been cited in the literacure.

The production of magnetophosphenes by the application of alternating fields is a well-accepted phenomenon. The major question, at this time, seems to be the mode of act ion responsible.

Medfum-to-high-strength, nonuniform steady-state flelds have wel1documentated effect upon rapldly growing tissues. Steady-state flelds with strengths approxituating the natural geomagnetic fleld are reported to produce alteracions in the pactern of blological cycles.

There is general agreement in the literature that whole-body exposure to flelds of a few thousand gauss should be avofied. This is primarily basto on the evidence that genetic effects occur in Insects and animals at such levels. However, there was no clear evidence on how serlous such effects would be for humans.

\section{References - Magnecic Field Fmfssions}

Barnothy, M. F., Blological Effects of Matnecic Flelds (Plenum Press, New York, 1964), Vo1. 1 .

Blologic and Clinical Effects of 1,ow Frequency Magnet ic and Electrdc FIelds (Thomas, Springfield, IL, 1974).

Kholodov, Yu. A., Effect of Electromagnet Ic and Magnetic Flelds on the Central Nervous System (CFSTI, Springfield, VA, 1967). 


\section{Rad lat lon}

\section{Lon1z1ng Radlation}

There are cwo kinds of blologlcal effects of lonizing radiation:

- Gentele effects, which are cranabitced to the offapting of the exposed Individual by wutations in the genes. Host experts agrec that any exposure to radiacion, Inciuding natural sources, can cause some genetic alceration, although long-tera genet fc changes are very difficult to seasure for small doses.

- Somncle effects, which lopalr the health or shorten the ilfe of chose exposed to radiation. There is considerable debate aver whecher exposure to very low radiacion levels causes somatic damge such ab cancer. Some selentists say that any dose of radiation is haraful. Others hold that low doses of rediation are not necessar 1 Iy horoful below a certaln throshold level. Unfortunately, the evidence is not ciearcut in deciding becween the two hypotieses. As a result. radiation exposure scandards wust be set as lou a possible while balancling benefics (such os wedical $x$ rays) against the probnbllity of hasm from low doses.

Genet 1c Effect - Concern over radiacion expoeste arlacs frow the fact that radlation 15 a sutagenli: agent. Each nes mutant added my result in harm co some descandane, and any addielona: number of defective individuals, of course, constitutes a greater burden to society. Sore Idea of the delicacy of the DNa molecule can be obcalned by considering that there are only 14 mg of DNh in the whole world, which took hundreds of centuries to evolve, and would strech from the earth to the moon.

. Evidence from the experiments with afce suggest that the frequency of nutations increases linearly with dose, but is also a function of dose rate. In male mice at high dose rates (90 rad/min) the doubling dose - the amount of radiation which would double the incidence of mutations - is approxiately $90 \mathrm{rem}$, whlle at lower measured dose rates ( 1 to $9 \mathrm{rad} / \mathrm{aln}$ ) the mutation frequency is one-third co one-quarter of this rate. Uaing these data, estimates have been made of the qutation frequencies and genetic deaths in man whlch may be coneributed by natural background radiation, diagnostic 
$x$ rays, and other sources of radiation. It has been estimated, and not without contention, that the number of genetic deaths introduced into the U.3. population each year by the genetically significant population dose from diagnogt ic $x$ rays - 5 area/y in 1964 - could range froa 1,100 to 44,000 deaths per year.

Somatic Effects - The neoplastic diseases, in particular leukemia and cancer, ore the somatic health effects that are of most concern. Neoplasia may be a long-delayed consequence of a large acute exposure or way follow clironic irradiation. It is by no means an inevitable result of any form of humar exposure. A single dose of 200 rads roughly triples one's chances of deraloping leuk. thia within a period of 10 years following irradiation. Low doses have been implicaced in the case of childhood leukemia and other neoplasms resulting frow diagnostic irradiation of the embryo during later stages of development. There is evidence that the leukemia risk of those ch!ldren exposed prenatally to doses of not more than a few rads is at least 40\% higher than the leukemla risk In children not so exposed.

When portlons of the body are irradiated with higher doses than can be administered to the whole body without deach, a variety of tumors may be produced, depending on the region being irradiated. Occupational exposure of the hands In earller decades produced many instances of skin lesions, precancerous changes, and malignancles. Neoplasms of the skeleton and imedincely surrounding tissues reguleing from long-term recention of radium in radium dial painters have received particular attention. The incldence of lung cancer in uranium miners is ancther example of a malignant neoplatele disease produced by radiation.

All forms of cancer can be increased by lonizing radiation, and one way co describe the phenomenon is either in termg of the dose required to double the spontaneous incidence rate of each cancer or, alternatively, as the incrense in incidence rate of such cancers per rad of exposure: 
For adultg

For youthful subjects (<20 years of age)

For Infants in utero

$$
\begin{aligned}
& \text { w00 rads as the doubling dosc. } \\
& \text { w\% increase in incidence rate } \\
& \text { per year per rad of exposure. }
\end{aligned}
$$

Between 5 and 100 rads as the doubling duse.

Between 1 and $20 \%$ Increase in incidence rate per year per rad of exposure.

26 rads as the doubling dose.

$217 \%$ increase in incidence rate per year per rad of exposire.

Cataracts - Suffictent exposure (on the order of 200 rads from $x$ or gamma rays) of the lens of the eye to ionlzing radiation may cause cataracts. Radiation-induced cataracts differ from radiation-induced leukemias and other eftects because they can be differentiated In many cases from the corcesponding defect due to other causes. A long latent pertod (5 years or more) exists between the time of exposure and the onset of the development of cararacts; however, for very high doses the interval can be reduced to months.

Growth and Development - At exposure levels substantially higher than those contemplated for occupational exposure, serlous effects on growth and development are demonstrable in animals and man. For example, Japanese children exposed to atomic bomb Irradiation showed some retardation of both growth and maturation. While growth and development have no spectal significance for adults occupationally exposed to radiation, the situation is markedly different in the prenatal child. As many as 24 types of major abnormalities have been reported among children irradiated in utero at dose levels between 50 and 200 rads.

Life Shortening - Although a prominent feature of radiation effects is the abllity of cells, tissue, and organs to recover, at least partially, from sublethal changes, some changes are induced at the cellular or $h \neq \bar{g} h e r$ level that may lead to late effects. Irreversible injury, which does not 
produce a recognizable, specific disease, may be expected to decrease the vitality or reserve capacity of these cells and organs in which it occurs. Thus, following sufflclent exposure to radiation, recognizable shortening of life span may be expected to occur Elther in association with increased or earlier incidence of speciflc disease or froth more general causes, at least superficlally simflar to premature aging. No life shortenting has been observed in humans, but It can be estimated from experimental effects in animals. The expected human lite shortening from nuclear plant effluents at thefr current release levels is estimated to be about 24 seconds. It Is inportant to compare this with the lffe-shortening effects of other agents (Tables 22 and 23 and Fig. 23).

Mutagenesis In its broadest sense - Including somatic and germinal effects, point mutagenesis, chromosome breakage (clastogenesis), and carcinngenesis - is curtently the dose-limiting blological criterion for setting radiation standards. Thls same dose-limiting genetic criterion will probably apply to many other energy effluents and to the spectrum of comnonly used dregs, food additives, and the chemicals of industry and agriculture. Understanding such effects and developing cools for their assay present a critical challenge to a technological society and are excicing areas for blological research.

Dur greatest capability for neasuring genetic effects is in microorganishs. There is a pressing need to relate such effects to higher organisms and to develop methods of sufficlent sensitivity and power to measure genetic damage directly in man.

\section{References - X Radiation}

Baines, E. C., "Ionizing Radiation," in The Industrial Environment - Its Evaluation and Control (National Institute for Occupdtional Safety and Health, Washington, DC, 1973), Chap. 29.

Gofman, J. W., and A. R. Tamplin, IEEE Trans. Nucl. Sctence NS-13, 1 (1970).

Hickman, R. G., Tritium in Nuclear Fusion Power, Lawrence Livermore Laboratory, Rept. UCRL-75546 (1974).

Report frum the National Research Councils Commitcee to Study the Long-Term Worldwide Effects of Multiple Nuclear Weapons Detonations, NAS-News Report (1975), P. 9 .

Schurr, S. H., Energy Research Needs, Resources for the Future, Inc., Washington, DC, Pub1. PB-207516 (October 1971). 
Table 22. U.S. population exposure from different sources of radiation, 1970.

\begin{tabular}{|c|c|c|c|c|c|c|}
\hline \multirow[b]{2}{*}{ Sontice } & \multicolumn{2}{|c|}{ 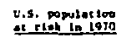 } & \multirow{2}{*}{ 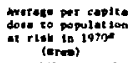 } & \multirow{2}{*}{ 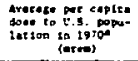 } & \multirow{2}{*}{ 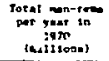 } & \multirow{2}{*}{ 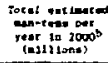 } \\
\hline & Perent & Mizlione & & & & \\
\hline \multirow{2}{*}{ 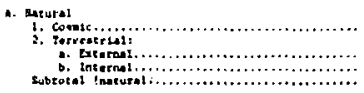 } & $10 c$ & 205 & 43 & 4s & D. 2 & 14.6 \\
\hline & $\begin{array}{l}100 \\
100 \\
100\end{array}$ & $\begin{array}{l}205 \\
205\end{array}$ & $\stackrel{+0}{\not 30}$ & $\underset{130}{130}$ & $\begin{array}{l}22.3 \\
3.1 \\
26.6\end{array}$ & $\begin{array}{l}19.3 \\
8.0 \\
61.9\end{array}$ \\
\hline \multirow{2}{*}{ 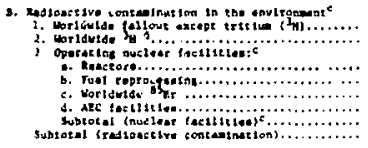 } & 100 & $\begin{array}{l}205 \\
209\end{array}$ & $\begin{array}{l}+.0 \\
0.01\end{array}$ & $\begin{array}{l}6.0 \\
0.07\end{array}$ & 0.07 & T.m \\
\hline & $\begin{array}{c}23 \\
0.3 \\
100 \\
0.6\end{array}$ & $\begin{array}{l}+1.60 \\
2.5 \\
203 \\
1.0\end{array}$ & $\begin{array}{l}0.0092 \\
0.113 \\
0.0003 \\
\therefore .1\end{array}$ & $\begin{array}{l}0.0021 \\
0.0008 \\
0.000 \\
0.01 \\
0.01 \\
+.1\end{array}$ & 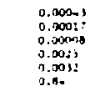 & $\begin{array}{l}3.016 \\
7.043 \\
7.012 \\
0.0013 \\
0.11 \\
2.9\end{array}$ \\
\hline \multicolumn{7}{|l|}{ 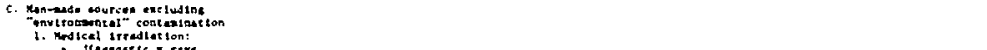 } \\
\hline 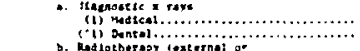 & $\because ?$ & $\begin{array}{lll}4 ., 51 \\
-3.44\end{array}$ & $250^{h}$ & $t e i_{i}^{n \cdot t}$ & in.: & -41 \\
\hline 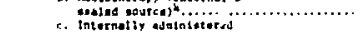 & 0.91 & 0.0251 & & $3.1^{\circ}$ & 2.) & \\
\hline 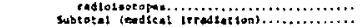 & s.? & 2.5 & & 1.1 & 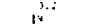 & $\because:$ \\
\hline 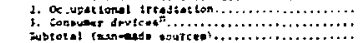 & $2: 0.6$ & $\operatorname{sos}^{0.11 \%}$ & $\therefore 2$ & $a_{12}^{0.2}$ & $a_{20}^{6, i n}$ & 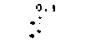 \\
\hline
\end{tabular}

Rele+ber does unlese atherwite nazed.

baind on an cetiented c.s, poputation in 20,00 equal to $121 \cdot 10^{\text {t. }}$

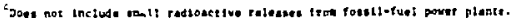

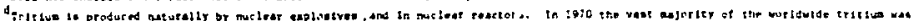

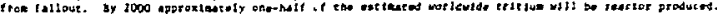

evignsa so miles af teactot alto.

twethin 100 ke of plent ase.

Gapilat loti at the in tobs.

The whole-body dowe to taken all the poned dise.

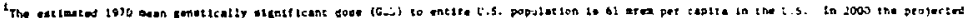

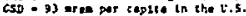

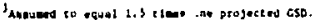

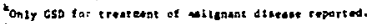

igbo sare.

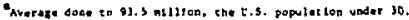

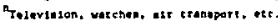

* Based on daca contained in a June 1971 draft, "Estimates of Ionizing Radiation Doses in the United States 1960-2000," Special Studies Group under the auspices of EPA.] 
Table 23. Comparison of estmated deaths due to man-made radtoactivicy in nuclearly stimulated ratural gas with gimilar escimates for ocher sources of radiation exposure of the public in the U.S.*

Sources of exposure

Estfmated deaths per mfllion Individuals exposed ${ }^{a}$

Natural background radiation ${ }^{b}$

Radioactivity in natural gas $c$

Natural (radon + daughters)

0.3

Man-made

0.03

Other man-made sources of radiation

Medical diagnostic $x$ rays

Pallout from nuclear weapons

0.4

Consurer devices

0.4

Industrial uses of radiation

$<0.2$

Power reactors

$<0.2$

abtalned by suming estimated somatic and genetic effects; therefore, some of these estimated deaths will occur among the exposad Individuals or the first generation of their offspring, but a large mafority (over Cऽ\%) will occur in succeeding generations.

$0.1 \mathrm{rem} / \mathrm{y}$.

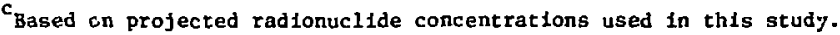

'[Rohewer, P. S., C. J. Barton, R. E. Moore, and S. V. Kaye, ThIrd Internationai Radiological Protection Association Meeting, Washington, DC (Steptember 1973).] 


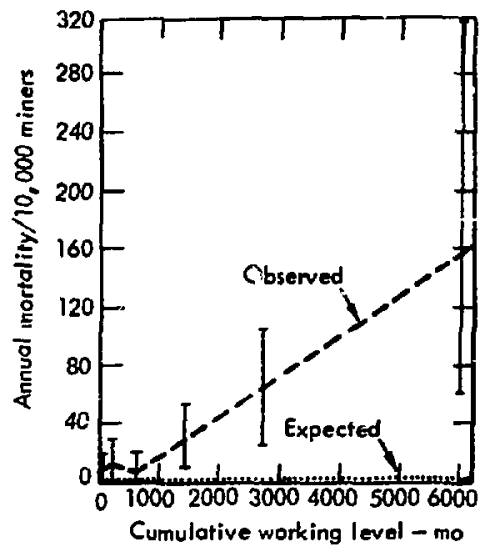

Fig. 23. Observed and expected annual luny cancer mortaljty per 10,000 miners and $95 \%$ confidence limits in relation to exposure. [Federal

Radiation Council, Washingtcn, DC, Rept. FRC No. 8, Revised (1967).] 


\section{Section III \\ Water Emuents}

\section{CHEMICAL FACTORS}

\section{Trace Metals}

Frum the standpoint of environmental effects, toxic elewents can be considered (1) noncritical, (2) toxic and relatively accessible, or (3) toxic, but very Insoluble or pery rare. Toxic elenents wove through the environwent under natural conditions by vay of the "geocycle" fron which the eleents become available to plants, antmals, and humans. Our activities provide neu sources of toxic elements that influence the peocycle and, consequently, the avallability of these element, to blological procrsses (FIg. 24 and Table 24 ).

One of the factors dete mining toxistiy concerns the chemical form to which the organtsm is exposed. Some Inorganic compounds are more or less toxic, depending on whether they are in the trivalent or pentavalent state; metallic-organics vary censiderably in their toxicity; and the form of an element present in high concentration in shrimp and other seafools is relatively noncoxic. For example, toxicities vary with the varlous for o of dercury, depending on whether it is wercury metal (quicksilver), Inorganlc mercury, or an organic compound containing the metal. Each form of a metal presents its own in ique spectrum of toxicity.

The metabolic activicy of microorganisms plays a significant part in the mobility of toxic elements in the environment. Microorganisms are exceedingly versatsle in the way they metabolize natural substances, and it can be sald that if they do not degrale a particular compound, then it is unlikely that highe: organisins will have the capacity to do so.

In the case of metals with no blological role, however, plants and antwals have generally fasled to develop a similar homeostat ic defense. Consequently, even small ameints of such toxic metals as lead and mercury are absorbed and readily accumulated by the organism. These metals, therefore, tend to be cumulative poisons.

The tendency for accumulation is reflected in the long biological half-iifa for these metals in humans - 2460 days for lead and 10 days for mercury. It should be recognized that the biological half-life has somewhat different meanings, depending on whether one is talking about the whole body, the liver, the bone compartment, or some other tissue. A long blological half-life can obviously be an lmportant factor influencing the toxility of a metal. 
Lead

Food furnishes 50 to $95 \%$ of the daily dose of lead. An urban dweller may take up an average of $20 \mu \mathrm{g} / \mathrm{day}$ from food, and $10 \mu \mathrm{g} / \mathrm{day}$ from air inhaled in the e1ty. The rural dweller recelves the same dose from food, presumably, but Inhales much less lead in the alr so that his uptake from the atmosphere is on.y $0.5 \mu \mathrm{g}$. Water contributes only a fraction of $1 \mu \mathrm{g} /$ day in elther case. Smoking 30 cigarettas per day may add another $10 \mu \mathrm{g}$ of lead to the dally dose. Plumbism, or lead poisoning, has been reported for adults with less than $80 \mathrm{\mu g} / 100 \mathrm{ce}$ of blood, while children have been reported to have lead poisoning with blood levels as low as $50 \mu \mathrm{g} / 100 \mathrm{cc}$. The average U.S. Citizen has about $25 \mu \mathrm{g} / 100 \mathrm{cc}$, with the range from 5 to $40 \mu \mathrm{g} / 100 \mathrm{cc}$ for persons not accupationally exposed. The margin of safety appears to be $10 \mathrm{w}$.

Chtldren and workers who are occupationally exposed are most subject to acute lead poisoning. From 6 to $10 \%$ of the children in lower soctoeconomic groups in Chicago have been found to have blood levels of $50 \mu \mathrm{g} / 100 \mathrm{cc}$ or more. In downstate citles, the Illinols Department of Public Health recently found 20 to $30 \%$ of such children had $40 \mu \mathrm{g} / 100 \mathrm{cc}$ blood lead, a value near the onset of overt symptoms of plumbisw. Children less than 2 years of age seem to be most susceptible. There is some evidence that certain individuals are most susceptible to lead polsoning due to genetic factor.

Mercury

The toxiclty of mercury is ettributed to its high affinity for sulfurcontalning compounds and its lesser affinity for organic 11gands. Interference by mercury in the synthesis and function of enzymes and other proteins can result in a varlety of adverse effects.

Alkyl mercury poisoning has somewhat different characteristics than other mercury polsoning. Symptoms may be dormant for weeks or months after acute exposure, and if brain damage occurs, effects may be Irreverstble. Inhalation of alkyl mercury can produce dryness and irritation in the nasopharynx and mouth.

Alkyl mercury polsonings have caused permanent neurological damage resulting in impaired vision and hearing, sensory loss in 1 imbs, ataxia, and tremor. Neurological disorders such as mental retardation and convulsive cerebral palsy have occurred in Infants whose mothers were exposed to methylmercury during pregnancy. Fetal nerve tissue may be especially sensitive to methylmercury. 
Ingestion of 1.0 to $2.0 \mathrm{~g}$ mercurlc chloride $1 \mathrm{~g}$ frequently fatal to human beings. It produces corrosion of the Intestinal tract, Infury to the kidnay, and ult imately death due to kidney fallure. The kidney appears to be the concentrator organ for Inorganic mercury.

Miscellantous Metallic Elements

A pecullar complication of the metal effluent plcture is that some of the metals cons dered to be dangerous are, in fact, needed in trace quantities by all plants and animals, including man. The metals vanadium, chromium, manganese, iron, cobalt, copper, zinc, selentum, molybdenum, and tin are usually incorporated into protelng and serve as catalysts which initiate or assist in many biological reactions. In addition to enzymes such as these, other biologically important molecules also contain essential metals. Ir antmals, for example, Iron is an integral part of the hemoglobin used for oxygen transport, while in plants the chlorophyll molecule contains magnestum. No fewer than 51 metals have been detected in the humar body, and undoubtedly some of these additional metals will eventually be shown to be necessary for 11fe.

Essential metals are, therefore, required in small quantities for the well being of living organisms. However, the same metals released to the enviromment in larger quantitles through man's activities or from natural sources can creace toxic conditions. 21nc and copper from mining operations, for example, can easily kill flsh and other aquatic organisus at very low concentrations, For essential metals, toxic levels may be 40 to $200 \mathrm{times}$ greater than that required for proper nutritional response.

Thus, for each of the metallic elements in the environment, to which all living organisms experience a finite level of exposure, the critical question of toxicity cannot be answered with a "yes" or "no," but by how much. What level of exposure to a specific metal is consistent with safety? In ozder to determine the levels that may be tolerated in alr, water and food, it is also necessary to know the level of unavoldable background exposure from all sources. 


\section{References - Trace Metals}

Berman, E., "Determination of $\mathrm{Cd}, \mathrm{Tl}$, and $\mathrm{Hg}$ in Biological Materials by Atomic Absorption," At. Abs NewslatLer 6, 57 (1967).

Braman, R. S., and C. C. Foreback, "Methylated Forms of Arsenic in the Environment," Science 182, 1247 (1973).

Buhler, D. H., Heavy Metals in the Envircnment (University of Oregon, Eugene, OR, 1973).

Dawson, G. W., The Chemical Toxicity of Elements, Battelle Pacific Northwest Laboratorles, Rochland, WA, Publ. BNWL-1815 (1974).

"EPA Promises Tough Enforcement of New Water Pollution Law," Chemical and Engineering News 50, 17 (1972).

Fishman, M. J., and D. E. Erdmanor, At. Abs. Newsletter 9, 88 (1970).

Goldwater, L. J., "Mercury in the Environment," Sc1. Amer. 224, 15 (1971).

Grant, N., "Mercury in Man," Environment 13, 2 (1971).

McKee, J. E., and H. W. Wolf, "Water Quallty Criterie," 2nd Editicn (State Water Works Resources Control Board, Sacramento, CA, 1963).

Meinke, W. W., and J. K. Taylor, Eds., Analytical Chemlstry, Key to Progress on National Problems (NBS Special Publication 351, WashIngton, DC, August 1972).

Merck Index, The, 8th Edition (Merck \& Co., Inc., Rahway, NJ, 1968).

Hoffitt, A. E., Jr., and R. E. Kupel, "A Rapld Method Employing Impregnated Charcoal and Atomlc Absorption Spectrophotometry from the Determination of $\mathrm{Hg}$ in Atmospheric, Biological and Aquatic Samples," At. Abs. Newsletter 9, 113 (1970).

Perry, H., "Environmental Aspects of Coal Mining," in Power Generation and Environmental Change, D. A. Berkowitz and A. M. Squires, Eds. (The MIT Press, Cambr Idge, MA, 1971).

Putman, J. J., "Wuicksilver and Slow Death," Natlonal Geograph1c 124, (October 1972).

Stah1, Q. R., Preliminary Air Pollution Survey of Mercury and Its Compounds, EPA Publication APTD 69-40 (USGP0, Washington, DC, 1969).

Taylor, R. T., and M. L. Hanna, Methylation of Plat Inum by Methylcobalarmin, Lawrence Livermore Laboratory, Rept. UCRL-76889 (1975). 
Table 24. Classification of elements according to thelr toxfcity. Elements omitted from this table should not be neglected in the environmental sense, For example, iodine and manganese are important elements, but they $f$ it mora than one category for the above classification.:

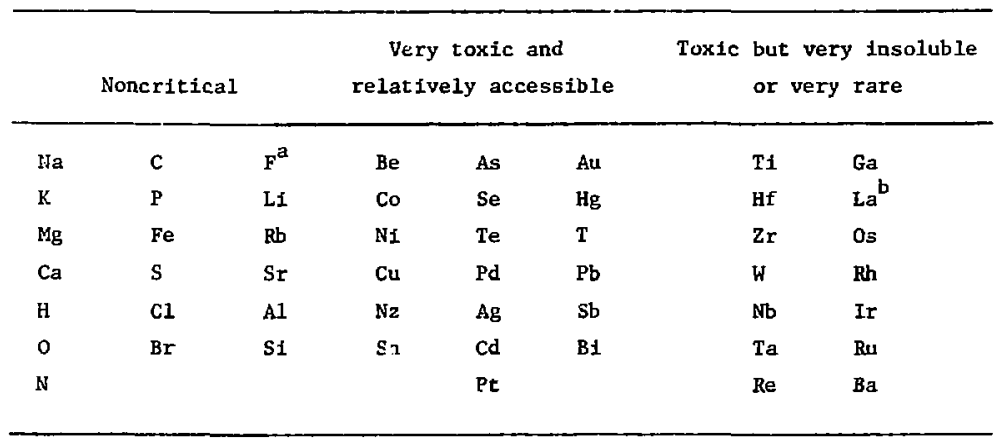

${ }^{a}$ Some may argue with this designation, but we do add fluoride to drinking water.

${ }^{b}$ All the lanthanides are very insoluble and some are very rare.

*[Wood, J. M., Science 183, 1049 (1974).] 


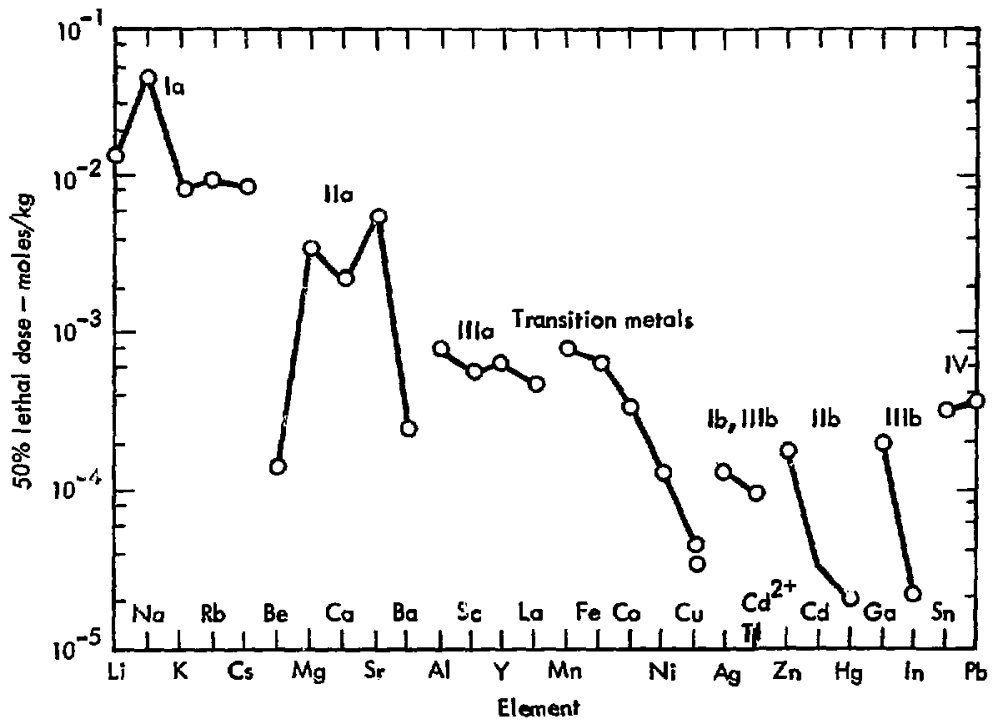

Fig. 24. A comparison of the lethal dose of various metal lons according to their position in the periodic table and their charge. Dose was adminlstered to mice Intraperitoneally. [Plerre Blenvenue, M. M., claude Nofre, and André Cler, "Toxicite Generale Comparee des Ions Mettaliques. Relation avec la Classification Periodique," Comp. Rend. 256, I043 (1963).] 


\section{RADIOLOGICAL FACTORS}

\section{Trit1um}

The effects of Interest of tritium are solely those due to the 1mpact of radioactivity on living things. Because of itg relatively short blological half-time and its relatively low energy, tritium is one of the least hazardous radionuclides produced in nuclear reactors. At the projected levels of activity for the year 2000, worldwide population exposure rates from ingestion of water would be sowrewhat less than $0.1 \mathrm{mrem} / \mathrm{yr}$ (compared with about $125 \mathrm{mrem} / \mathrm{yr}$ from all natural sources).

\section{References - Tritlum}

Butler, H. L., and J. H. LeRoy, "Observation of Blologlcal Half-Life of Trit1um," Health Physics 11, 283 (1965).

Moglifss1, A. A., M. W. Carter, and R. Lleberman, "Long Term Evaluation of the Blological Half-Life of Trittum," Health Physics 21, 57 (1971).

Hylle, K. F., H. A. Blgler, and G. R. Grove, "Blological Half-Life of Tritlum," Health Physics 9, 911 (1963). 


\section{other Radioact 1uity.}

The health effects of radioactive materials ingested by humans depend in a complicated way on the half-life of the radionuclide, its mode of decay, the organ in which it is concentrated, the quantity present, the half-iffe (residence time) of the element in the body, and the age of the person, as well as other factors (Fig. 25).

Iodine and strontium isotopes, in particular, are concentrated by the body: infants and children are the critical sectors of the population. Iodine Is taken up by the thyrold gland; strontium is incorporated in newly formed bone. Cesium, on the other hand, is distributed in the soft tissues.

Although we know that high concentrations of radiolodine can destroy the thyroid function and high concentrations of radiostrontium could produce bone cancer or changes in bone struxcure, no data are available as to the effects on man of extremely low concentrations such as would result from exposure of human populations to maximum permissible concentrations (MCPs) of ${ }^{131} \mathrm{I}$, ${ }^{90} \mathrm{Sr}$, or ${ }^{137} \mathrm{Cs}$.

The main radiological impact of radiostrontium in man is due to 1 ts biochemical resemblance to calctum and tts Iong half-life. When Ingested, some of the strontium becomes fixed in the bones where it rematrs for a long time. Its subsequent decay produces radiation dose directly at the site (the $2.27-\mathrm{MeV}$ highest-energy beta of the daughter $90_{\mathrm{Y}}$ has a maximum range of only about $1.0 \mathrm{~g} / \mathrm{cm}^{2}$ ). Because of this, the recommended MCPs in air and water are quite sma11.

\section{References - Other Radioactivity}

Forbes, G. B., "Significant Isotopes in Foods," in Conference on the Pediatric significance of Peacetime Radloactive Fallout, supplenent to Pediatrics 41 [1], Part II, 20 ? (January 1968). 


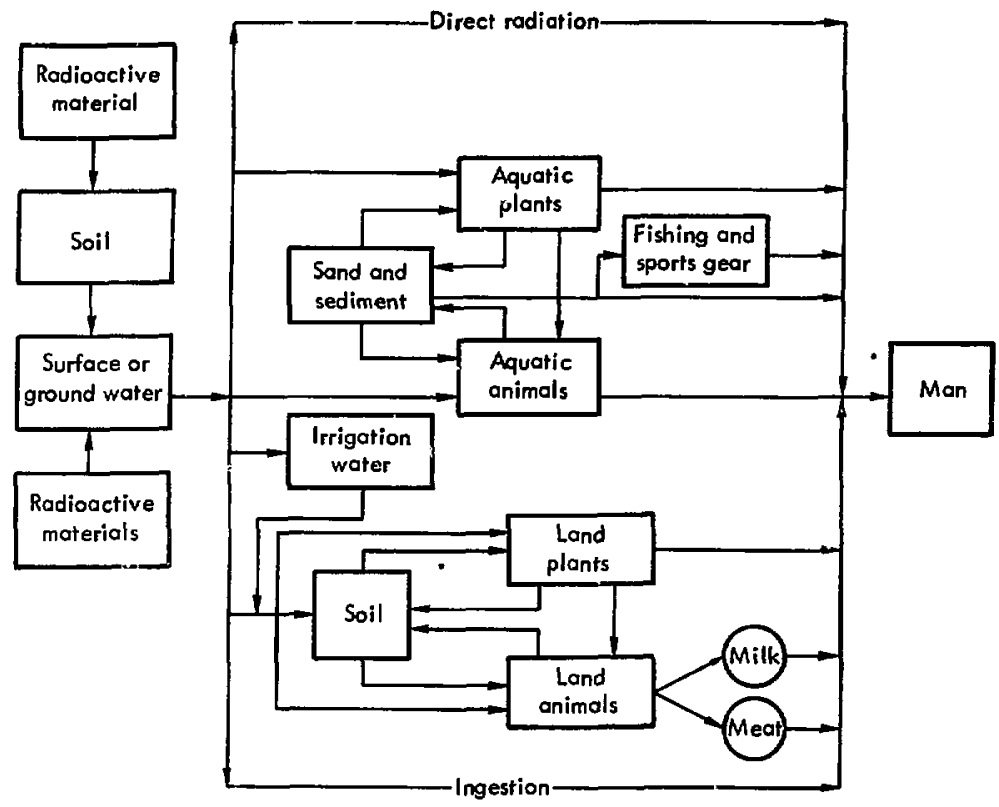

Fig. 25. Pathways between radioactive materials released to ground and surface waters (including oceans) and man. [Instrumentation for Environmental Honttoring, RAD-REA Nuclear Reactors, Append ix $C$, p. 34, Lawrence Berkeley Laboratory, University of California, Berkeley, CA.] 


\section{PHYSICAL FACTORS}

\section{Thermal Inputs to Water}

Fleld studies made to date have demonstrated that as water temperature rises it holds less dissolved oxygen. Also, as the water temperature rises aquatic organisms require more dissolved oxygen in order to maintain a normal existence, or to live at all.

The cemperature requirements of a given species of fish vary throughout 1ts Iife history. The requirements differ for spawning and the development of eggs and fry. The sensitivity or tolerance of aquatic organisms to temperature changes or levels also varies with age, size, and season.

Lethal high and low temperatures vary widely for different spectes. The difference in upper lethal levels for different species vartes as much as $32^{\circ} \mathrm{F}, \mathrm{e} . \mathrm{g} ., 41^{\circ} \mathrm{F}$ for the goldfisis and $75^{\circ} \mathrm{F}$.or the pink salmon. Water tenperatures do not have to reach lethai levels, however, to wipe out a species. Temperatures which favor competitors, predators, parasites, and diseases can destroy a species at levels far below those whlch are lethal. As water temperatures increase, bacterial action and the so-called natural purification process are speeded up. Then, oxygen depletion may occur in summer in certain areas where dissolved oxygen conditions are sitisfactory In ather seasons.

\section{References - Thermal Inputs to Hater}

Afr/Water Pollution, Staff Report, 12, 393 (October 7, 1974).

Brooks, N., "Energy and the Envitronment," Eng. and Sc1. 24, (January 1971).

Coutant, C., Blological Aspects of Therma1 Pollution, $t$. Entrainment and Discharge Canal Effects, CRC Critical Reviews In Environmental Control, Vol. 1, Issue 3, November, 1970.

Energy lnternational (1971), p. 22.

HIckman, R. G., Lawrence Livermorn Laboratory, Rept, UCRL-75546 (1974).

John, James EA., "Thermal Pollution: A Potential Threat to our Aquatic Envirunment, Environmental Affairs. 1. 12\} 287 (1971),

Miller, A., et al., Use of Steam Electclc Power Plants to Provide Thermal Energy to Urban Areas, Oak Ridge Nat Ional Laboratory, Oak Ridge, $T N$, ORNL-HUD-14 (1971).

Morris, D., Future linergy Demand and Its Effect on the Enviranment, Rand Corporation, Santa Nonica, CA, Report No. R-1098-NSF (September 1972). 
Parker, F., and P. Krenke1, Physical and Engineering Aspects of Thermal Pollution, CRC Press, Cleveland, Ohis (1970).

Schuss, S. H., Energy Research Needs, Resources for the Future, Inc., Washington, DC, Pub1. PB-207516 (October 1971).

Selected Materials on Environmental Effects of Producing Electric Power, Chapters $V$ and VIII, U.S, Congress, Jolnt Committee on Atomic Energy, 91 st Congress, lst Session, Hashington, DC (August 1969).

Water Quality Criter1a, National Technical Advisory Comit tee on Wate: Quality Criteria, a report to the Secretary of the Interior (USGPO Washington, DC, 1968).

Woodson, R. D., "Cooling Towers," Sc1. Amer. 224, 7r. (May 1971). 


\section{Section IV}

\section{Land Use}

\section{CHEMICAL, FACTORS}

\section{Acid Fallout}

Effects toay or may not Include changes in the leaching rates of nutrients from plant follage, changes in the leaching rates of soll nutrients, effects on predator/prey relationships and effects on the metabolism of organisms.

Increased input of acid to soil way lead to increased leaching of calcium and other nutrient elements. Acidity is also correiated with humus sontent. Ii causal, then the acid would help return minerals. Although such losses would be small for some ecosystems and unlikely so result in any significant short-term damage to arable land, they do represent other added stresses to the ecosystem. During the growing season in forested areas most of the acid rain impinges directly on the follage where fon exchange accurs. Thus the rain that reaches the forest scil is far less actd than the incident precipitation.

Serious fish mortality, particularly early age classes in salmonolos, has been attributed directly to Increased acidity from precipitation. Similar acidification of aquatic ecosystems must be occurring in other parts of the world, but it is unrecognized or is often confounded by other sources of pollution.

So long as there is sufficient lime, it is impossible to harm soll with excess $\mathrm{SO}_{2}$ over and above crop needs. It merely causes conversion to calclum sulfate. Nor does any excess of sulfur cause an equivalent lime loss in the dralnage. Most of the loss of lime from arable land is caused by solution of bicarbonate resulting from the high $\mathrm{CO}_{2}$ concentration in the air in the soll. Sulfate, whether from $\mathrm{SO}_{2}$ or from fertflizers, serves partly to displace bicarbonate. Aphagnum muss actually takes minerals out of rain, replacing them by hydrogen (acid) so that the runoff from such land is naturally acid. Lakes In such high-rafnfall districts, such as kiorth Wales and southwest Sweden, have been naturally acid for centuries. 
The lakes in Sweden which have sreated the alarm are those in the high-rainfall area of the southwest, with very little buffer capacity, and conseguently low productivity. There are perhaps 3000 of them, medium to small, among Sweden's 100,000 lakes. It has been known, and practiced, for almost 50 years that the lakes aust be IImed to give a reasonable yield of fIsh.

\section{References - Acid Fallout}

Erickson, E., "The Fate of SO and NO it the Atmusphere," in Power Generarion and Environmental Change, D. A. Berkoticz and A. M. Squares, Eds. (The MIT Press, Cambridge, MA, 1971).

Ferenbaugh, R. W., Environmental Affairs, 745 (1975).

Schfermeirer, F., and 1. Niemeyer, Large Power Plant Effluent Study, Vol. 1 Instrumentation, Procedures, and Data Publications, Department of Health, Education and Helfare, Natfonal Air Pollution Conirol Agency, Wa shington, DC (1970). 


\section{Solid Effluent}

Solfd effluent dumped il the iand is aesthetically objectionable. Some components of spent shale effluent and sludge effluent from pover plant scrubbers are significantly water soluble and carcinogenic, indicating the need to guard against uncontrolled leaching. Tests have indicated that water after Intfoate contact with spent shale will be highly alkaline and contain high concentrations of calcium, sodium, and potassium in the form of sulfates, whether or not mineral decomposition has occurred in the retorting process.

Additional salinity in the Colorado River system and at Hoover Dam would come from: leaching of spent shale both during waste pile buildup and after revegetation; reinjection of mine water and upward movement and surface discharge of saline waters; accidental release of low-quaisty mine water, including fallure of evaporation ponds; and any return flows of sallne water. Contributions of salts from these sources could increase the salinity at Hoover Dam to as much as $50 \%$ (1140 mg/liter).

Erosion of shale oil disposal piles (both durfing bulldup and after revegetation) and of off-site construction areas such as utility corridors, transportation networks, and town site expansion will lead to increased sediment loads in local streams.

Up to 10 gallons of water w111 be produced from each ton of shale during the retorting process, and this water will have sizable quantities of polycyclic aromatic hydrocarbons, some of which will be carcinogentc. The water could be treated to remove the contaminants, but industry officials propose using the water to wet down and thus stabilize the shale refuse piles. This procedure, of course, would add to the carcinogenic material already in the piles.

The wastewater from the processed shale is different from additional Industrial water which also will cause a waste disposal problem after being used in the overall mining, retorting, and disposal operation. For that operation, scme three barrels of Industrial water will be needed for every barrel of shale ofl produced. To ranchers and farmers in the arid West, the water may be more preclous than the ofl. 
How readily the native grasses and shrubs can grow on refuse piles is a question that will largely determine the environmental effects of solld waste. In the case of shale oll refuse dumps, many small plots show that at least three nattve grasses grow profusely, after watering heavily for the first year, using commerclal fertilizer, and illching. Two native shrubs have also grown, but so far (1975) none of trie woody species used as browse by deer have achieved maturity.

\section{References - Solld Effluent}

Environment 16 (July/August 1974). 


\section{RADIOLOGICAL FACTORS}

\section{High-Level Storage}

The principal concern with respect to high-level radiation effluent is accidental leakage into the blosphere. Modes by which this might occur include: (a) leakage from liquid-storage tanks; (b) rupture of solid-waste containers en route from processing plant to burlal site: (c) migration of radioactive materlals during storage. Such accldents could result in local or widespread contamination of populated areas depending on the circumstances and whether or not radioactive materials had entered the hydrosphere.

Atoms of medium atomic weight, formed by fisston of uranium or plutonium, form one category of effluent. The isotopes ${ }^{90} \mathrm{Sr},{ }^{137} \mathrm{Cs}$, and to a lesser extent ${ }^{85} \mathrm{Kr}$, are the main elements; ${ }^{93} \mathrm{Zr},{ }^{129} \mathrm{I},{ }^{135} \mathrm{cs}$, and others are much less Important. In 700 years, less chan one ten-millionth of these radioactive elements remains.

The other category of radioactive effluent consists of the actinides (the elements actinium, thorium, uranium, neptunium, plutonium, and so os), which are formed not by fission but by neutron absorption into the original uranium (or thorfum) fuel. All are very toxic and most have long half-lives - for example, about 25,000 years for ${ }^{239} \mathrm{Pu}$, the most abundant transuranium actinide, which is formed either in conventional light-water reactors or in proposed 1iquid-metal fast breeder reactors (FIgs. $26-28$ ).

The actinides cause effluent management difficulties at two distinct points in the nucleas fuel cycle; some are carried over with the fission products during nuclear fuel reprocessing, and some highly dilute plutonium wastes w1ll appear from fuel manufacturing plants. Two of the most important things about this category of effluent are that (1) the offending actinides are relatively toxic and (2) alchough initlally far less radloactive than the fission products, they become dominant at 500 years because of their much longer half-lives.

\section{References - High-Level Storage}

Evans, R. D،, Physical, Biological, and Administrative Problems Associated with the Transportation of Radioactive Substances, Revised edition (NAS-NRC,

Smeets, J., Health Implications of the Storage of Radioactive Substances on and in the Ground (Luxembourg, European Communities, Netherlands). 


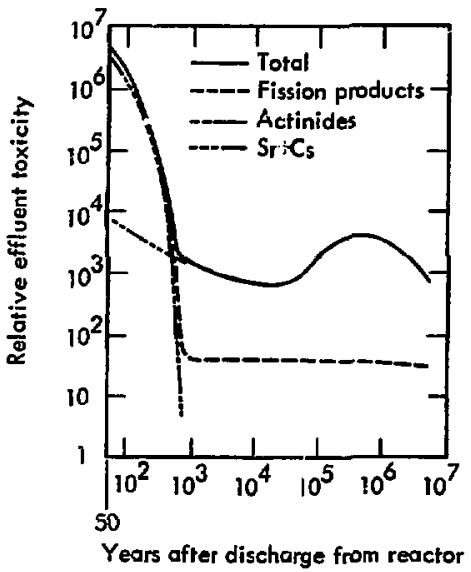

FA.g. 26. Toxicity of effluent from light-water reactors, for an equilibrium fuel cycle, with $99.5 \%$ removal of uraniun and plutonium. Each metrlc ton of fuel is assumed to deliver a total thermal energy of 33,000 WW/day during its operating Iffetime. The turn-up at 106 years arises from growth of daughter products not present in the original material, which ig not in decay equilibrium. [Kubo, A. S., and D. J. Rose, Science I82, 1205 (1973).] 


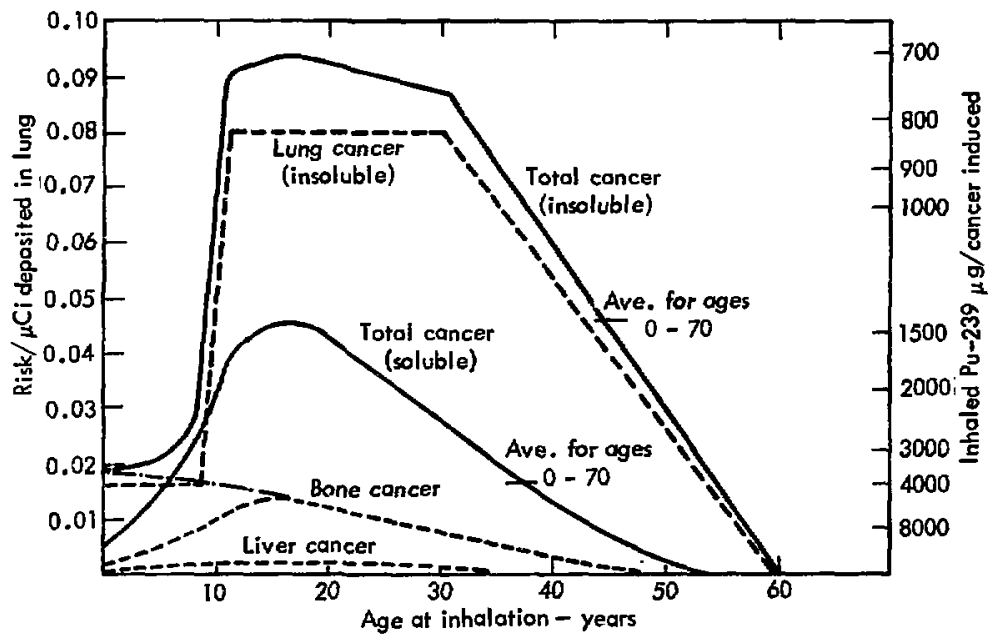

Fig. 27. Risk of radiation-Induced cancer death per $\mu$ CI of Pu deposited in lung vs age at inhalation. The scale at the right is the lethal dose (i.e., dose/cancer induces) converted to mass of $P u$ and corrected for deposition efficiency of Inhaled Pu (25\%). The curves for bone and liver cancer have been corrected at young ages for reduced breathing rate in children. The curves without this correction are used for calculating effects of infection in the bloodstream and are shown by the dot-dash lines. [t+sh, B. R., et al., ORNL-NSIC-74, P. 108.1 


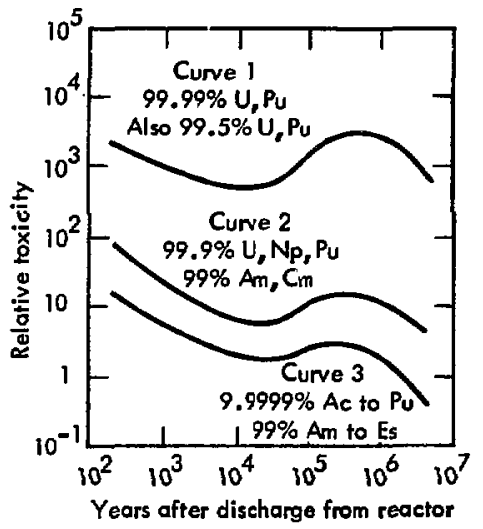
Fig. 28. Toxicity of effluent from 1ight-water reactors, for different degrees of actinfde extraction. [Kubo, A. S., and D. J. Rose, Science 182, 1205 (1973).]


PHYSICAL. FAC'LORS

\section{Strip- and Surface-kining Activities}

Adverse

The ecology of streams In cunoff areas is disturbed by acidic materlals and stlting, and the land-damaging and air emission effects can extend for miles beyond the immediate area of mining operat fons (rable 25). When area mining has been completed and not reciaimed, the land is left corrugated with a pattern of long parallel ridges and valleys caused by erosion and land slides.

In California, extracting 2 million tons of gravel along the Russian River has resulted in altering the channel of the river, caving in banks, washing away adjoining land, and wuddying water downsteaw, harming both fishery and recreational resources. The Sonoma Councy Wacer Agency reports that gravel extraction also imperils the water supplies for 150,000 people In Sonoma and northern Marin counties.

Mine tallings and low-grade ore left untreated from an abandoned copper mine near Keswick in Shasta Councy, California, leached polsonous copper residues into the Sacramento RIver. After construction of Shasta Dam cut the water fiow, the increased concentrations of copper became lethal to migrating salmon. As a result, the Federal government spent more than a million dollars trying to impede the flow of polsoned water, but the project has been only partially successful and additional water has had to be released from Shasta Dam to dilute the copper further.

Near the city of Sonora, In Northern California, milling wastes and tallings from a recently abandoned asbestos wiae have produced dangerous dust particles. While the mine dumps are not near populated areas, the local official responsible for the enforcement of air emission standards has not ruled out potential health hazards to surrounding comunitles.

Benefictal

In the western U.S., some surface mines have exposed gzound-water sources and made water available where none existed before. This water has proved invaluable to 1j.vestock and wijdife. At some surface-mining operations along mountainsides, the pits lmpound surface runoff from torrential rains, minimize the sidiment load of streams draining the area, and effect considerable ground-water recharge as well. 
In California, plles of dredge cailings are quite permeable. However, because of thetr Irregular conformation, they undoubtedly inhibit surface runoff to a greater degree than the original slopes, thus making some contrlbution to flood control and ground water recharge.

In Alaska, dredge mining for gold has destroyed the permafrost and the resulting callings and wined areas are considered premium property for resldencial and industrial developaent.

Surface wining has created any opportunities to develop recreational wreas where none existed before. Wacer In the form of small ponds or lakes, and the spoll piles themselves, frequencly provide a pleasant copographlc change in areus of virtually flac land. Examples may be found in flat coastal areas in such iscaces as Kansas, Illinois, Indiana, Ohio, and california.

\section{References - Serip- and Surface-Hining Activicles}

Mom's Impact on Environment. T. R. Detwyler, Ed. (Mctraw-hill Book Co. New Fork. 1971), p. 348 .

Prifrio, M. H. Call fornla Jaurnal, (Noveraber 1974). 
Table 25. The coal miner and his health - Eacts and figures (1971), Daphne A. Roe, M. D.*

Total number of l.1ving coal miners

250,000

Total number of penstoners

67,203

Thtal number of pensioners recelving income from social security viz: 51,330 on ald age pension and 8,756 on disability pension

60,086

Total number of pensloners recelving Blark Lung jenefits

17,901

Toral number of pensioners receiving work nen's compensation

Total number of penstoners recelving UNWA pensions 66,658

Total number of penstoners employed in nonmining jobs

Total number of miners in work force 182,797

Total number of working miners recelving Black Lung or total

disability benefics

Total number of working miners receiving workmen's compensation

for infurles sustained in mining accidents

Average monthly income from old age penston

Average tonthly income from disability pension

Average monthly income from Black Lung pension

210.21

Average monthly income from WCB pension

151.10

Average wonthly income from unW pension

150.00

\section{Annual est imates of health costs (based on January 1971 figures)}

It has been estimated that disability penstons cannot be regarded as occupational health costs, because they are awarded for a varlety of diseases which are not occupational.

Occupational health custs include:

Black Lung benefits for penstoners

$$
\begin{array}{r}
\$ 45,155,630.52 \text { p.a. } \\
3,006,843.84 \text { p.a. } \\
5,880,207.60 \text { p.a. } \\
3, ! 8 j, 600.40 \text { p.a. }
\end{array}
$$

Black Lung benefits for talners

WCB payments for pensioners

WCB payments for thiners

Total

$\$ 58,026,282.36$ p.a.

*[Cumputed from Report of United Mine Workers of America Welfare and Retirement Fund, year ending June 30, 1971.] 


\section{Power-PIant Siting}

A principal concern regarding the siting of power plants and transuission systems is the Incolapatibility of these systems with competitive land uses, particularly residential and recreational uses. The aesthetics of current designs leaves much to be decired, and residential communties fear for the effect on property values; the public in general and environmentalists in particular deplore the despoiling of wilderness and otherwise uncontaminated natural areas. Unfortunately, the most desirable regions for these altemative land uses, which tend to be the areas of interface between land and water, are also the most desirable for the siting of power plants because of the avallability of cooling water.

A transmission system passing chrough wild and forested areas requires brush and tree clearing along the right-of-way in order to provide physical clearance for the transmisston lines and access for Inspection and matntenance activity. This clearing plus the use of herbicfdes for maintenance of the right-of-way are matters of considerable environmental concern.

long-term safety of nuclear power plants is concerned with the permissible radiation levels in the vicinity of the power-plant sites and the disposal of the long-ifved radioactive wastes resulting from the nuclear fuel reprocessing cycle. Short-term concerns pertain to the destructive release of radiation in reactor accidents or acts of God.

Doutts still exfst that backup cooling systems and reactor containment designs are adequate to handle potentfal emergencies.

\section{References - Power-Plant Sting}

Environmental Effects of Producing Electric Power (Joint Commitree on Atomic Energy, USGPD, Washington, DC, 1969).

Power-Plant SitIng and Environmental Protection (House Committee on Interstate and Foreign Commerce, USGPO, Washington, DC, 1971).

Power Plant Siting, Its Impact on System Planning and the Environment (IEEE, New York, 1971). 
Hydroelectric Dang

Congsiuction of a large dam in southern Indla has ralsed the water table in the surrounding district making the soll wore alkaline and thereby upsetting the trace element balance in the diet of local inhabitants. The traglc consequence is genu valgum, a crippling bone disease affecting young paople.

Downstream from the Aswan Dax, water now flows year round concributing to increased snall population and schistosoniosis.

\section{References - Hydroelectrlc Dam:}

Agarwal, A. K., New Sclent1st 65, 260 (January 30, 1975). 